\title{
Three-dimensional protein structure determination by high-resolution solid-state NMR spectroscopy
}

\author{
Dissertation \\ zur Erlangung des Doktorgrades \\ der Mathematisch-Naturwissenschaftlichen Fakultäten \\ der Georg-August-Universität zu Göttingen
}

vorgelegt von

Adam Lange

aus Münster

Göttingen 2006 
D7

Referent: Prof. Dr. Tim Salditt

Korreferent: Prof. Dr. Christian Griesinger

Tag der mündlichen Prüfung: 18. April 2006 


\section{Contents}

1. Introduction 1

2. Distance constraints from CHHC / NHHC experiments 9

2.1 Theoretical background 9

2.2 Solid-state NMR spectroscopy 12

2.3 Quantum-mechanical simulations 15

2.4 Molecular-dynamics based structure calculations 18

2.5 Analysis of zero-quantum ${ }^{1} \mathrm{H}-{ }^{1} \mathrm{H}$ polarization transfer 20

2.6 Analysis of double-quantum ${ }^{1} \mathrm{H}-{ }^{1} \mathrm{H}$ polarization transfer 27

2.7 Molecular 3D structures from CHHC / NHHC constraints 28

2.8 Conclusions 33

3. The 3D structure of uniformly labeled kaliotoxin 43

3.1 Introduction 43

3.2 Solid-state NMR spectroscopy 44

3.3 Resonance assignments and structural constraints 46

3.4 Molecular-dynamics based structure calculations 48

3.5 Results 51

3.6 Conclusions $\quad 54$

4. The 3D structure of epothilone B 61

4.1 Introduction $\quad 61$

4.2 Solid-state NMR spectroscopy $\quad 62$

4.3 Resonance assignments and structural constraints 64

4.4 Molecular-dynamics based structure calculations 65

4.5 Results 65

4.6 Conclusions $\quad 69$ 
5. Structural characterization of a high-affinity toxin / potassium channel complex

5.1 Introduction $\quad 73$

5.2 Sample preparation $\quad 76$

5.3 Electrophysiological experiments and binding studies $\quad 76$

5.4 Solid-state NMR experiments and resonance assignments 77

5.5 Structure calculations and model building 82

5.6 Results 85

$\begin{array}{ll}5.7 \text { Conclusions } & 90\end{array}$

6. Summary 95

Bibliography $\quad 99$

$\begin{array}{ll}\text { Appendix } & 101\end{array}$

$\begin{array}{ll}\text { Acknowledgements } & 115\end{array}$

$\begin{array}{ll}\text { CV } & 117\end{array}$ 


\section{Introduction}

The Protein Data Bank (PDB) of the Research Collaboratory for Structural Bioinformatics (RCSB) is a repository for three-dimensional (3D) structures of proteins and nucleic acids ${ }^{1}$. It currently holds 35343 entries (28-Feb-2006). The vast majority of the deposited structures has been obtained by applying X-ray crystallographic techniques $^{2}(\sim 85 \%)$ and solution-state nuclear magnetic resonance (NMR) spectroscopy ${ }^{3,4}(\sim 15 \%)$. These biophysical methods require protein single crystals and protein solubility, respectively. While X-ray structures of proteins usually exhibit a slightly higher accuracy and precision than NMR derived structures, NMR spectroscopy has the advantage that it probes not only structure but also dynamics. In many cases knowledge of the dynamics of a protein is a prerequisite if its function is to be understood.

A large fraction $(\sim 25-30 \%)$ of the human genome encodes membrane proteins. Whenever molecules or information are transferred across the cell membrane, membrane proteins such as ion channels ${ }^{5}$ or $\mathrm{G}$ protein-coupled receptors ${ }^{6}$ are involved. Despite a strong incentive to structurally characterize this class of proteins only few membrane protein structures have been elucidated so far (28-Feb-2006: $93^{7}$ ). In addition to difficulties in producing adequate amounts of purified protein, most membrane proteins resist crystallization. Since membrane proteins are inherently insoluble also the application of solution-state NMR spectroscopy is challenging and requires the solubilization of the membrane protein with the help of amphiphilic detergents. In contrast, protein samples that lack both long-range order and solubility are amenable to a solid-state NMR spectroscopic investigation ${ }^{8}$. In principle, this allows for the structural characterization of a membrane protein reconstituted into phospholipid bilayers. Here, the resulting proteoliposomes closely mimic the natural environment of a membrane protein, the cell membrane.

The NMR spectrum of a solid-phase sample is usually dominated by anisotropic interactions such as chemical shift anisotropy (CSA) and homo- and heteronuclear dipolar couplings. Different from NMR spectroscopy on isotropic solutions where the CSA is averaged to an isotropic value and the dipolar couplings to zero, these interactions lead to a significant line-broadening of the solid-state NMR spectrum. 
The fast macroscopic rotation of the sample around an axis tilted at $54.7^{\circ}$ from the external magnetic field (magic-angle-spinning, $\mathrm{MAS}^{9}$ ) in combination with heteronuclear decoupling techniques is often sufficient to obtain high-resolution ${ }^{13} \mathrm{C}$ and ${ }^{15} \mathrm{~N}$ solid-state NMR spectra (see Figure 1.1). Compared to ${ }^{1} \mathrm{H}$ these nuclei are characterized by a significantly smaller natural abundance and gyromagnetic ratio $\gamma$. The natural abundance of these rare-spin low- $\gamma$ nuclei in proteins can be increased to almost one by isotopic labeling. For this purpose proteins are, for example, expressed in Escherichia coli cell cultures grown in a medium supplemented with ${ }^{13} \mathrm{C}_{6}$-Dglucose and ${ }^{15} \mathrm{~N}-\mathrm{NH}_{4} \mathrm{Cl}$.

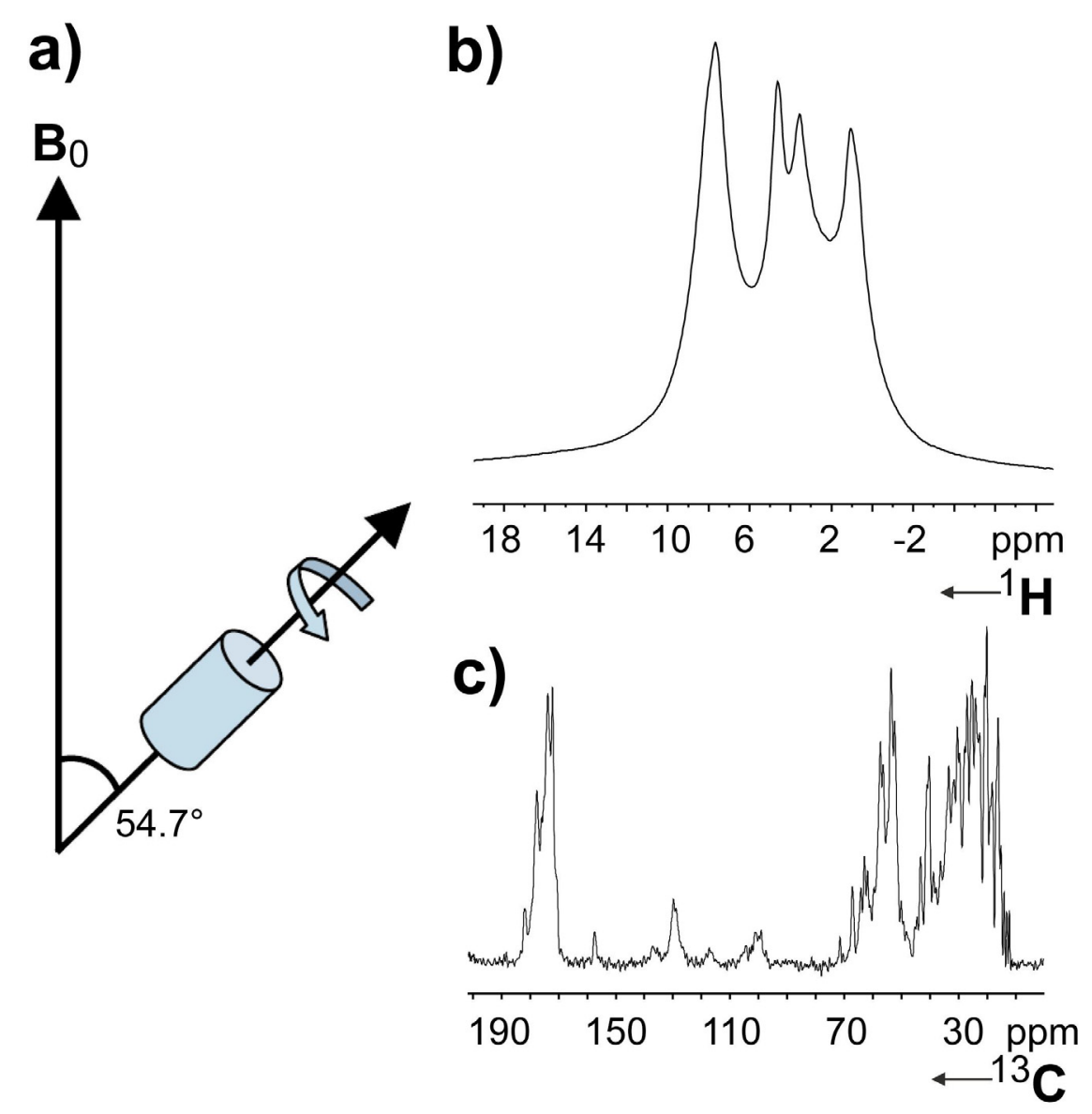

Figure 1.1. a: Magic-angle-spinning (MAS) establishes high-resolution conditions for solid-state NMR spectroscopic investigations of solid-phase polypeptides. b: ${ }^{1} \mathrm{H}$ spectrum of the tri-peptide Ala-Gly-Gly at $30 \mathrm{kHz}$ MAS. c: ${ }^{13} \mathrm{C}$ cross-polarization $(\mathrm{CP})$ spectrum of the 85 -amino acid protein $\mathrm{Crh}$ at $11 \mathrm{kHz}$ MAS. Both spectra were recorded on a $600 \mathrm{MHz}\left({ }^{1} \mathrm{H}\right.$ resonance frequency) wide-bore spectrometer (Bruker Biospin, Germany).

Direct ${ }^{1} \mathrm{H}$ solid-state NMR spectroscopy is usually restricted to samples that show either residual mobility or possess only a limited set of resonances. In solid proteins 
the high gyromagnetic ratio and abundance of the ${ }^{1} \mathrm{H}$ nuclei result in a network of strong homonuclear dipolar couplings and consequently in homogenously broadened lines. Nevertheless, the high ${ }^{1} \mathrm{H}$ Boltzmann equilibrium polarization and short $\mathrm{T}_{1}$ relaxation time can be utilized in a cross-polarization ${ }^{10,11}(\mathrm{CP})$ experiment to enhance the sensitivity of ${ }^{13} \mathrm{C}$ and ${ }^{15} \mathrm{~N}$ spectroscopy.

The 3D structure determination of soluble proteins is based on the measurement of a large set of ${ }^{1} \mathrm{H}-{ }^{1} \mathrm{H}$ distances ${ }^{3}$. This can be readily achieved by means of twodimensional nuclear Overhauser effect spectroscopy (NOESY) ${ }^{12}$. If not prohibited by spectral overlap these spectra yield distance constraints for all ${ }^{1} \mathrm{H}-{ }^{1} \mathrm{H}$ pairs in the protein simultaneously ${ }^{3}$. Because direct ${ }^{1} \mathrm{H}^{-}{ }^{1} \mathrm{H}$ correlation spectroscopy in the solidstate is not possible in general (as discussed above) distance measurements in solidphase proteins have so far been restricted to ${ }^{13} \mathrm{C}$ and ${ }^{15} \mathrm{~N}$ nuclei. As described in detail in chapter 2, the collection of a sufficient set of structurally relevant distances between these nuclei requires either the preparation of multiple samples or the recording of a large set of NMR experiments. On the other hand, ${ }^{1} \mathrm{H}-{ }^{1} \mathrm{H}$ correlation spectroscopy in the solid-state can be combined with high-resolution spectral dimensions $^{13-15}$. This approach has been used to quantitatively characterize domain sizes of spatially heterogeneous materials ${ }^{16,17}$. It has been demonstrated that using these indirect detection schemes combined with short ${ }^{1} \mathrm{H}-{ }^{1} \mathrm{H}$ mixing times allows for the simultaneous detection of a large set of ${ }^{1} \mathrm{H}-{ }^{1} \mathrm{H}$ correlations in a solid-phase $\operatorname{protein}^{18}$, the detection of inter-molecular constraints in self-aggregated chlorophyll ${ }^{19}$, and the assignment of ${ }^{15} \mathrm{~N}$ resonances in polypeptides ${ }^{20}$. The combined results from these CHHC, NHHC and NHHN experiments are equivalent to the information that can be obtained by performing a NOESY experiment in solution-state NMR spectroscopy. Furthermore CHHC, NHHC and NHHN experiments can be performed with a double-quantum ${ }^{1} \mathrm{H}-{ }^{1} \mathrm{H}$ mixing sequence ${ }^{18}$. In the resulting two-dimensional spectra the sign of the cross-peaks depends on the number of protons involved in the transfer pathway. Thus, a distinction between relay and direct cross-peaks becomes possible. This is equivalent to rotating frame Overhauser effect spectroscopy (ROESY) in solution-state $\mathrm{NMR}^{21}$. In chapter 2 a theoretical framework for the quantitative analysis of CHHC, NHHC and NHHN experiments is developed. For this purpose, it will be investigated how the cross-peak intensities depend on the ${ }^{1} \mathrm{H}-{ }^{1} \mathrm{H}$ 
distance of interest and on the used mixing time, magnetic field strength, MAS frequency and mixing sequence. Such a comprehensive analysis is mandatory if indirectly detected ${ }^{1} \mathrm{H}-{ }^{1} \mathrm{H}$ correlations are to be included in a molecular-dynamics based structure calculation. Chapter 2 also exemplifies in two simple cases how ${ }^{1} \mathrm{H}-{ }^{1} \mathrm{H}$ constraints can be combined with chemical shift derived torsion angle constraints and how ambiguities from methylene and methyl protons can be dealt with.

a
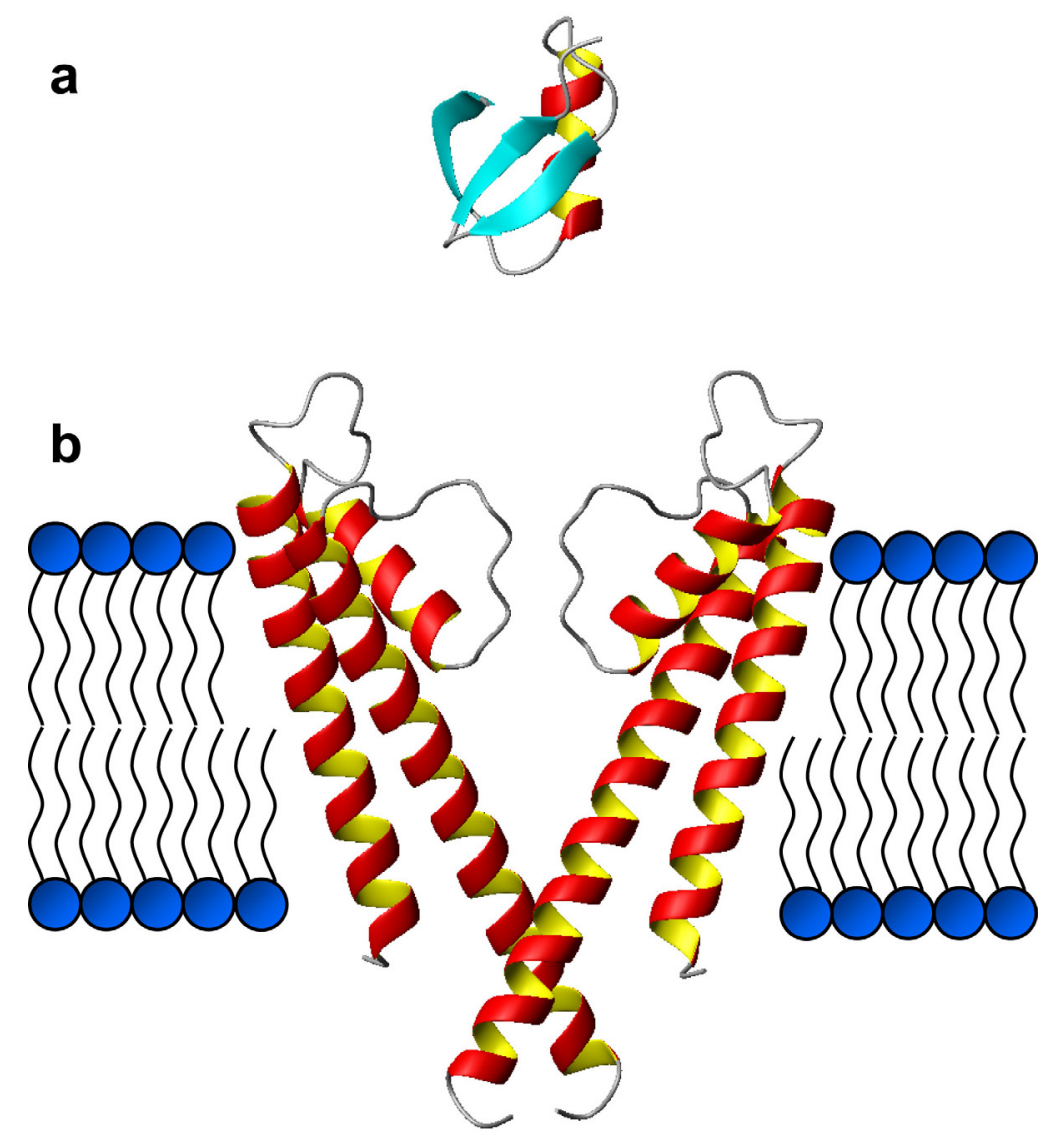

Figure 1.2. Ribbon diagrams of a: kaliotoxin from the venom of the scorpion Androctonus mauretanicus mauretanicus as determined by ${ }^{1} \mathrm{H}$ solution-state NMR spectroscopy ${ }^{22}$ (PDB entry: $2 \mathrm{KTX}$ ) and b: the prokaryotic potassium channel KcsA from Streptomyces lividans as determined by X-ray crystallography ${ }^{23}$ (PDB entry: $1 \mathrm{~K} 4 \mathrm{C}$ ). For the sake of clarity only two monomers of the homotetrameric protein are shown.

In chapter 3 a concept for de novo 3D structure determination of uniformly $\left[{ }^{13} \mathrm{C},{ }^{15} \mathrm{~N}\right]$ labeled solid-phase proteins by solid-state NMR spectroscopy is introduced. The approach is based on the combination of indirectly detected ${ }^{1} \mathrm{H}-{ }^{1} \mathrm{H}$ constraints from CHHC and NHHC experiments and chemical shift derived backbone torsion angles. It 
is applied to the 38-residue peptide kaliotoxin (KTX, see Figure 1.2a). Similar to a structure calculation process based on NOESY experiments, ambiguous cross-peaks from CHHC / NHHC spectra are assigned in an iterative cycle of assignment and structure calculation. The concept will be compared to an alternative approach for $3 \mathrm{D}$ structure determination based on an alternating ${ }^{13} \mathrm{C}-{ }^{12} \mathrm{C}-{ }^{13} \mathrm{C}-\ldots$ labeling pattern ${ }^{24}$.

To investigate whether the introduced approach is also applicable to the study of a uniformly $\left[{ }^{13} \mathrm{C}\right]$ labeled non-peptidic molecule chapter 4 contains results on the compound epothilone $\mathrm{B}$, that binds with high affinity to polymerized $\alpha \beta$-tubulin ${ }^{25}$. Here, also the influence of the inter-molecular hydrogen-bond formation in crystalline material on the solid-state NMR chemical shift is investigated. While these experiments were performed on a powder-crystalline sample of free, i.e. unbound, epothilone $\mathrm{B}$, they provide a basis for future studies of its bioactive conformation and binding mode to polymerized $\alpha \beta$-tubulin.
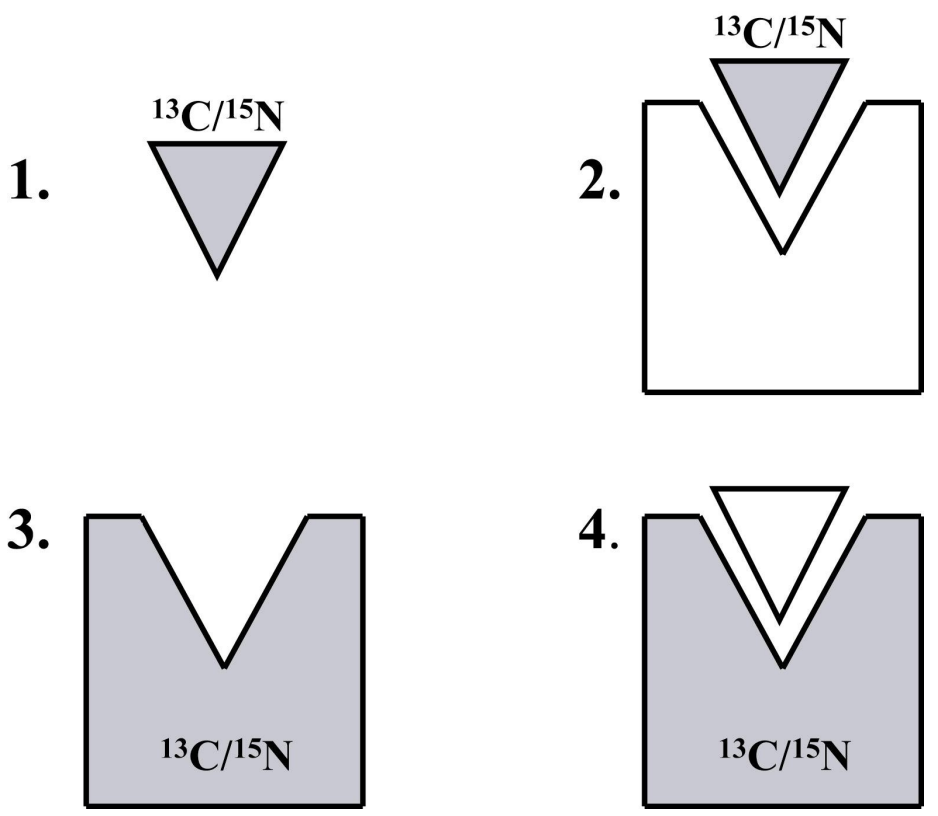

4.

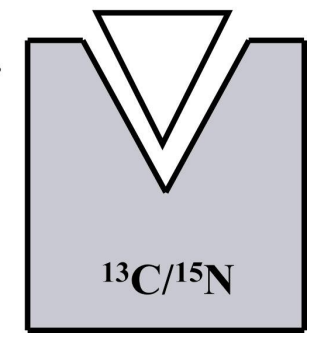

Figure 1.3. Labeling scheme for KTX (triangle) / KcsA-Kv1.3 (box) samples. Uniformly $\left[{ }^{13} \mathrm{C},{ }^{15} \mathrm{~N}\right]$ labeled molecules are depicted in grey. Non-labeled molecules are shown in white.

Finally, chapter 5 describes how solid-state NMR spectroscopy can be used to investigate the high-affinity binding $\left(K_{\mathrm{d}}=0.3 \mathrm{nM}\right)$ of KTX to the potassium $\left(\mathrm{K}^{+}\right)$ channel KcsA-Kv1.3 ${ }^{26}$. While structural information is available for the structurally 
related $\mathrm{K}^{+}$channel KcsA from Streptomyces lividans (as determined by X-ray crystallography ${ }^{23}$; PDB entry: $1 \mathrm{~K} 4 \mathrm{C}$; see Figure 1.2b) and for KTX in solution (based on ${ }^{1} \mathrm{H}$ solution-state NMR spectroscopy ${ }^{22}$; PDB entry: 2KTX) the exact mode of interaction remains elusive. In fact, up to now no 3D structure of any high-affinity toxin / ion channel complex has been reported. To study interaction and 3D structure of the two binding partners in its complex by solid-state NMR, experiments are performed on four differently labeled samples (see Figure 1.3). By comparing chemical shift assignments for free (Figure 1.3: sample 1) and bound (sample 2) KTX, residues that are involved in the interaction with the ion channel can be identified. Similarly, a comparison of chemical shifts of free (sample 3) and KTX-bound (sample 4) ion channel reveals the KTX binding pocket of KcsA-Kv1.3. Furthermore, conformation-dependent chemical shifts of samples 2 and 4 in combination with CHHC and NHHC experiments can be utilized to probe the 3D structure of the two molecules in the complex. Thus, possible conformational changes that accompany the complex formation can be detected. 
1. Berman, H. M. et al. The protein data bank. Nucleic Acids Research 28, 235$242(2000)$.

2. Huber, R. A structural basis of light energy and electron transfer in biology (Nobel lecture). Angewandte Chemie-International Edition in English 28, 848869 (1989).

3. Wüthrich, K. NMR of proteins and nucleic acids (Wiley Interscience, New York, 1986).

4. Cavanagh, J., Fairbrother, W. J., Palmer, A. G. \& Skelton, N. J. Protein NMR spectroscopy, principles and practice (Academic Press, San Diego, 1996).

5. Hille, B. Ion channels of excitable membranes (Sinauer Associates Inc., Sunderland, MA, 2001).

6. Lundstrom, K. H. \& Chiu, M. L. G protein-coupled receptors in drug discovery (Taylor and Francis, Boca Raton, 2006).

7. http://www.mpibp-frankfurt.mpg.de/michel/public/memprotstruct.html

8. Baldus, M. Correlation experiments for assignment and structure elucidation of immobilized polypeptides under magic-angle-spinning. Progress in Nuclear Magnetic Resonance Spectroscopy 41, 1-47 (2002).

9. Andrew, E. R., Bradbury, A. \& Eades, R. G. Nuclear magnetic resonance spectra from a crystal rotated at high speed. Nature 182, 1659-1659 (1958).

10. Pines, A., Gibby, M. G. \& Waugh, J. S. Proton-enhanced NMR of dilute spins in solids. Journal of Chemical Physics 59, 569-590 (1973).

11. Hartmann, S. R. \& Hahn, E. L. Nuclear double resonance in the rotating frame. Physical Review 128, 2042-2053 (1962).

12. Jeener, J., Meier, B. H., Bachmann, P. \& Ernst, R. R. Investigation of exchange processes by two-dimensional NMR spectroscopy. Journal of Chemical Physics 71, 4546-4553 (1979).

13. Zhang, S., Meier, B. H. \& Ernst, R. R. Polarization echoes in NMR. Physical Review Letters 69, 2149-2151 (1992).

14. Wu, X. L., Zhang, S. M. \& Wu, X. W. Two-stage feature of Hartmann-Hahn cross-relaxation in magic-angle sample spinning. Physical Review B 37, 98279829 (1988).

15. Zhang, S., Meier, B. H. \& Ernst, R. R. Local monitoring of proton spin diffusion in static and rotating samples via spy detection. Solid-state Nuclear Magnetic Resonance 1, 313-320 (1992).

16. Mulder, F. M., Heinen, W., van Duin, M., Lugtenburg, J. \& de Groot, H. J. M. Spin diffusion with ${ }^{13} \mathrm{C}$ selection and detection for the characterization of morphology in labeled polymer blends with MAS NMR. Journal of the American Chemical Society 120, 12891-12894 (1998).

17. Wilhelm, M., Feng, H., Tracht, U. \& Spiess, H. W. 2D CP/MAS ${ }^{13}$ C isotropic chemical shift correlation established by ${ }^{1} \mathrm{H}$ spin diffusion. Journal of Magnetic Resonance 134, 255-260 (1998).

18. Lange, A., Luca, S. \& Baldus, M. Structural constraints from proton-mediated rare-spin correlation spectroscopy in rotating solids. Journal of the American Chemical Society 124, 9704-9705 (2002).

19. de Boer, I., Bosman, L., Raap, J., Oschkinat, H. \& de Groot, H. J. M. 2D ${ }^{13}$ C${ }^{13} \mathrm{C}$ MAS NMR correlation spectroscopy with mixing by true ${ }^{1} \mathrm{H}$ spin diffusion reveals long-range inter-molecular distance restraints in ultra-high magnetic field. Journal of Magnetic Resonance 157, 286-291 (2002). 
20. Wei, Y. F. \& Ramamoorthy, A. $2 \mathrm{D}{ }^{15} \mathrm{~N}-{ }^{15} \mathrm{~N}$ isotropic chemical shift correlation established by ${ }^{1} \mathrm{H}-{ }^{1} \mathrm{H}$ dipolar coherence transfer in biological solids. Chemical Physics Letters 342, 312-316 (2001).

21. Bothner-By, A. A., Stephens, R. L., Lee, J. M., Warren, C. D. \& Jeanloz, R. W. Structure determination of a tetrasaccharide - Transient nuclear Overhauser effects in the rotating frame. Journal of the American Chemical Society 106, 811-813 (1984).

22. Gairi, M. et al. 3D structure of kaliotoxin: Is residue 34 a key for channel selectivity? Journal of Peptide Science 3, 314-319 (1997).

23. Zhou, Y. F., Morais-Cabral, J. H., Kaufman, A. \& MacKinnon, R. Chemistry of ion coordination and hydration revealed by a $\mathrm{K}^{+}$channel-Fab complex at 2.0 Å resolution. Nature 414, 43-48 (2001).

24. Castellani, F. et al. Structure of a protein determined by solid-state magicangle-spinning NMR spectroscopy. Nature 420, 98-102 (2002).

25. Heinz, D. W., Schubert, W. D. \& Höfle, G. Much anticipated - The bioactive conformation of epothilone and its binding to tubulin. Angewandte ChemieInternational Edition 44, 1298-1301 (2005).

26. Legros, C. et al. Generating a high-affinity scorpion toxin receptor in KcsAKv1.3 chimeric potassium channels. Journal of Biological Chemistry 275, 16918-16924 (2000). 


\section{Distance constraints from CHHC / NHHC experiments}

\subsection{Theoretical background}

Solid-state nuclear magnetic resonance (NMR) has recently made considerable progress in providing structural information in systems such as surface bound peptides $^{1}$, membrane $e^{2-6}$, or fibrous proteins ${ }^{7-14}$ that are difficult to study by solutionstate NMR or X-ray crystallography. The vast majority of these studies (for recent reviews, see refs ${ }^{15-19}$ ) involve rare-spin nuclei such as ${ }^{13} \mathrm{C}$ or ${ }^{15} \mathrm{~N}$. In polypeptides, the strongest dipolar couplings between these nuclei are predominantly intra-residual or sequential (i.e., couplings between a nucleus of residue $i$ and a nucleus of residue $j$ with $|\mathrm{i}-\mathrm{j}|=1)$. In general, solid-state NMR can probe only the strongest dipolar couplings. Weaker couplings are truncated in the presence of stronger couplings. Therefore, non-trivial structural information from medium-range $(1<|\mathrm{i}-\mathrm{j}|<5)$ and longrange $(|\mathrm{i}-\mathrm{j}|>4)$ dipolar couplings is only obtainable if the stronger couplings are effectively reduced. This can be achieved by advanced labeling approaches ${ }^{20-24}$ and/or by the application of specifically designed NMR pulse schemes that selectively reintroduce the dipolar coupling of interest while the stronger couplings remain averaged out by magic-angle-spinning (MAS) ${ }^{25-28}$. In uniformly labeled polypeptides, ${ }^{13} \mathrm{C}-{ }^{13} \mathrm{C}$ or ${ }^{13} \mathrm{C}-{ }^{15} \mathrm{~N}$ distance constraints between 3 and $6 \AA$, which are of central importance for defining backbone and side chain conformation, have thus far only been determined using such chemical shift selective transfer methods ${ }^{25,26,29}$. In this case, each individual distance constraint necessitates the recording of at least one NMR data set. Even in small peptides, the complete 3D structural characterization can hence require a considerable number of NMR experiments, making structural investigations in larger systems impractical.

In contrast to dipolar couplings between rare-spin nuclei ${ }^{30-36}$, even the strongest ${ }^{1} \mathrm{H}-{ }^{1} \mathrm{H}$ dipolar couplings provide valuable structural details and, in many cases, lead to a remarkably accurate description of the three-dimensional fold of the molecule of interest $^{37}$. It is therefore not surprising that biomolecular structure determination by solution-state NMR relies profoundly on the detection of short $(<5 \AA)$ proton-proton distance constraints ${ }^{37-39}$ which can be identified with considerable reliability ${ }^{37,40-42}$. Here, a variety of radio frequency (r.f.) mixing schemes (including NOESY $\mathrm{N}^{38,41,43}$ and 
ROESY ${ }^{44-46}$ experiments) are available to simultaneously detect a large set of ${ }^{1} \mathrm{H}-{ }^{1} \mathrm{H}$ contacts as cross-peak intensities within a standard multi-dimensional correlation experiment.

Ideally, a similarly large set of ${ }^{1} \mathrm{H}-{ }^{1} \mathrm{H}$ distance constraints, read out in a single, 2D correlation experiment, could provide the basis for determining the three-dimensional arrangement of a molecule in the solid-state. Here, the ability to directly detect ${ }^{1} \mathrm{H}-{ }^{1} \mathrm{H}$ contacts is strongly influenced by the residual proton line width. Due to the network of strong homonuclear dipolar couplings the proton lines are homogenously broadened. Only in systems with a limited set of ${ }^{1} \mathrm{H}$ resonances or in the presence of residual internal mobility, line-narrowing techniques such as multiple-pulse decoupling ${ }^{47}$ or ultrafast $\mathrm{MAS}^{48}$ are sufficient to probe proton-proton interactions in high spectral resolution. In many biophysical applications, complications arise due to the large number of ${ }^{1} \mathrm{H}$ resonances and due to the limited spectral dispersion. For this reason, indirect detection schemes that encode ${ }^{1} \mathrm{H}-{ }^{1} \mathrm{H}$ dipolar couplings on rare-spin nuclei are mandatory. They have previously been used in the context of ${ }^{1} \mathrm{H}$-spin counting $^{49,50}$ and for 2D rare-spin correlation spectroscopy ${ }^{51-54}$.

In polypeptides, a variety of non-trivial distance constraints can be detected if ${ }^{15} \mathrm{~N}$ or ${ }^{13} \mathrm{C}$ evolution periods are used and if the ${ }^{1} \mathrm{H}-{ }^{1} \mathrm{H}$ mixing time is restricted to the initial rate regime ${ }^{53}$. If constraints obtained from these CHHC / NHHC 2D experiments are to be supplemented in the framework of a de novo three-dimensional structure determination, the transfer intensities must be examined in more detail. In addition, such attempts require a principle understanding of the polarization transfer dynamics within a proton network in the solid-state. As the general driving force of ${ }^{1} \mathrm{H}-{ }^{1} \mathrm{H}$ transfer in the solid-state, dipolar interactions between neighboring proton spins have thus far been described as a deterministic, quantum-mechanical (QM) transfer mechanism $^{48,49}$ or as a dissipative spin diffusion process ${ }^{55-57}$. An appropriate theoretical description furthermore not only depends on the details of the ${ }^{1} \mathrm{H}-{ }^{1} \mathrm{H}$ mixing but also on the time scale for which ${ }^{1} \mathrm{H}-{ }^{1} \mathrm{H}$ transfer is allowed to take place. For example, results of mixing times in the order of ms have been analyzed within the concept of a classical exchange process with a uniform diffusion coefficient ${ }^{51,52}$. As will be shown below, a simple extension of this model for shorter mixing times ${ }^{54}$ or a 
QM analysis successfully utilized at ultrafast MAS conditions ${ }^{48}$ is not possible in general. Instead, a detailed analysis of the influence of experimental parameters such as the $\mathrm{B}_{0}$ field, MAS rate, and the type of r.f. mixing scheme is mandatory.

In the following, experimentally detected cross-peak build-up curves from $\mathrm{CHHC}$ and NHHC experiments are analyzed using three different theoretical models.

(a) Spectral spin diffusion ${ }^{55-57}$. Here, the cross-peak signal intensity is described by an exponential buildup:

$$
I_{12, z}(t)=1-\exp \left(-t / T_{S D}\right)
$$

In the static case (i.e., without MAS), the spin-diffusion time constant $T_{\mathrm{SD}}$ is given by the ${ }^{1} \mathrm{H}-{ }^{1} \mathrm{H}$ distance $\mathrm{r}_{12}$ of interest and the zero-quantum (0Q) line-shape function, evaluated at the isotropic chemical shift difference $\Delta$ of $I$ spins 1 and 2 :

$$
\frac{1}{T_{S D}}=\frac{1}{(2 \pi)^{2}}\left(\mu_{0} / 4 \pi\right)^{2} \frac{\hbar^{2} \gamma_{I}^{4}}{r_{12}^{6}} J^{0 Q}(\Delta)
$$

To lowest approximation, equation 2.2 remains valid under MAS and/or the presence of an r.f. scheme assuming a modified 0Q-line-shape function $J^{0 Q, R F}\left(\Delta, \omega_{R}\right)^{57}$.

(b) Two-spin approximation. Within the simulation environment GAMMA ${ }^{58}$, a dipolar coupled ${ }^{1} \mathrm{H}-{ }^{1} \mathrm{H}$ two-spin system was monitored under the influence of MAS for all considered r.f. mixing schemes (see section 2.3). Isotropic and anisotropic ${ }^{1} \mathrm{H}$ chemical shielding interactions were taken from the literature ${ }^{59-61}$.

(c) It was also investigated whether the concept of a standard diffusion coefficient ${ }^{51,52}$ would adequately explain the experimental findings.

In sections 2.5 and 2.6 of this chapter it will be shown that an adequate theoretical analysis of ${ }^{1} \mathrm{H}-{ }^{1} \mathrm{H}$ transfer rates generally depends on the details of the NMR 
experiment. A comprehensive examination of the transfer dynamics in uniformly labeled model compounds leads to a general framework in which ${ }^{1} \mathrm{H}-{ }^{1} \mathrm{H}$ transfer rates can be related to inter-nuclear distances for the considered proton-proton mixing schemes and MAS rates. Section 2.7 of this chapter exemplifies how these distance constraints can be utilized in the context of a molecular-dynamics based structure calculation to determine the $3 \mathrm{D}$ conformation of a uniformly $\left[{ }^{13} \mathrm{C},{ }^{15} \mathrm{~N}\right]$ labeled biomolecule in the solid-state.

\subsection{Solid-state NMR spectroscopy}

$\mathrm{U}-\left[{ }^{13} \mathrm{C},{ }^{15} \mathrm{~N}\right]$ labeled samples of L-Valine and L-Histidine $\cdot \mathrm{HCl}$ were purchased from Cambridge Isotope Laboratories (CIL, Andover, MA). U- $\left[{ }^{13} \mathrm{C},{ }^{15} \mathrm{~N}\right]$ labeled Ala-GlyGly was chemically synthesized as described previously ${ }^{62}$. L-Histidine $\cdot \mathrm{HCl}$ and AlaGly-Gly were recrystallized from aqueous solutions at $10 \%$ dilution using standard procedures.

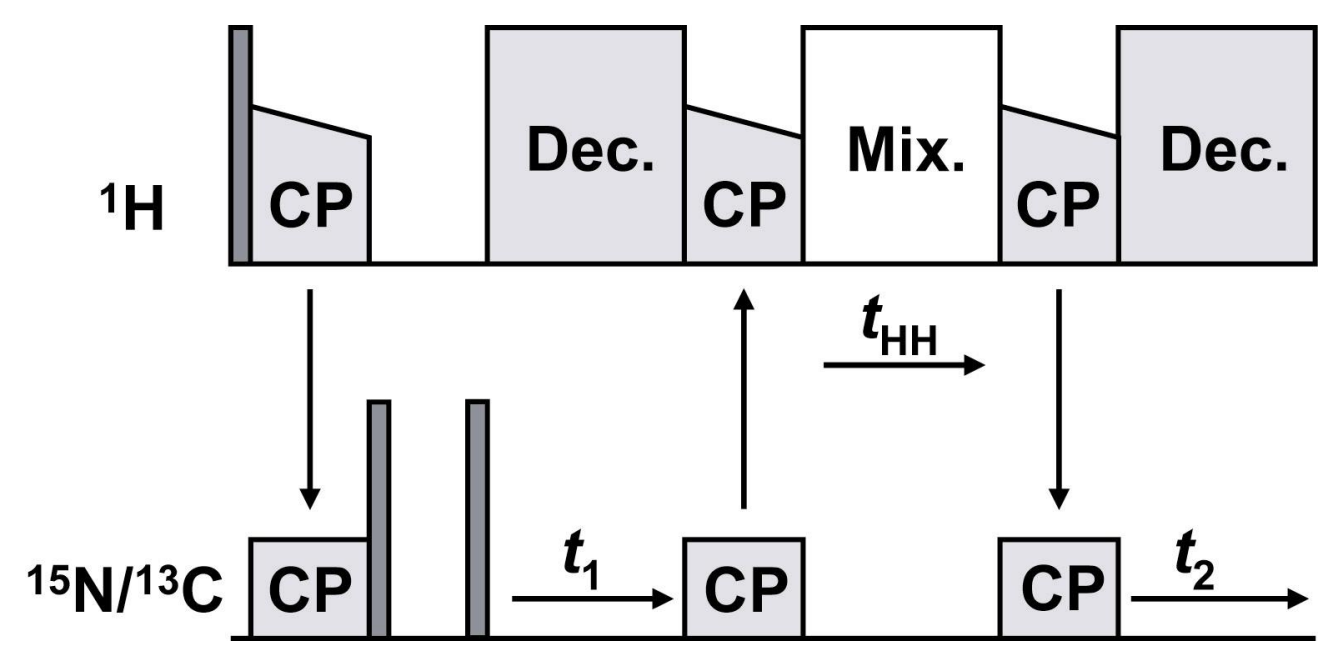

Figure 2.1. Generic two-dimensional pulse scheme to observe ${ }^{1} \mathrm{H}-{ }^{1} \mathrm{H}$ polarization transfer in two spectral rare-spin dimensions ${ }^{53} .{ }^{1} \mathrm{H}-{ }^{1} \mathrm{H}$ contacts are encoded during a two-dimensional ${ }^{15} \mathrm{~N}-{ }^{13} \mathrm{C}(\mathrm{NHHC})$ or ${ }^{13} \mathrm{C}-{ }^{13} \mathrm{C}$ (CHHC) correlation experiment.

All NMR experiments were conducted on $9.4 \mathrm{~T}\left({ }^{1} \mathrm{H}\right.$ resonance frequency: $\left.400 \mathrm{MHz}\right)$ or $14.1 \mathrm{~T}\left({ }^{1} \mathrm{H}\right.$ resonance frequency: $\left.600 \mathrm{MHz}\right)$ wide-bore instruments (Bruker Biospin, Germany) using $2.5 \mathrm{~mm}$ and $4 \mathrm{~mm}$ triple-resonance $\left({ }^{1} \mathrm{H},{ }^{13} \mathrm{C},{ }^{15} \mathrm{~N}\right)$ MAS 
probes. The generic two-dimensional CHHC / NHHC mixing scheme ${ }^{53}$ is depicted in Figure 2.1 and involves rare-spin evolution and detection periods. Unless stated otherwise, heteronuclear contact times $t_{\mathrm{HN}}$ and $t_{\mathrm{HC}}$ were set to 200 and $100 \mu \mathrm{s}$, respectively. The length of the z-filter was set to $3 \mathrm{~ms}$. Phase sensitive 2D spectroscopy was established by means of TPPI phase cycling ${ }^{38}$.

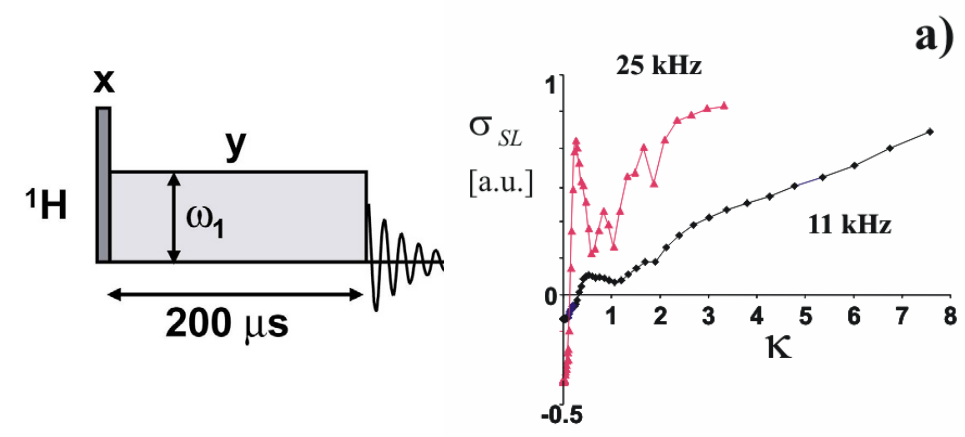

a)

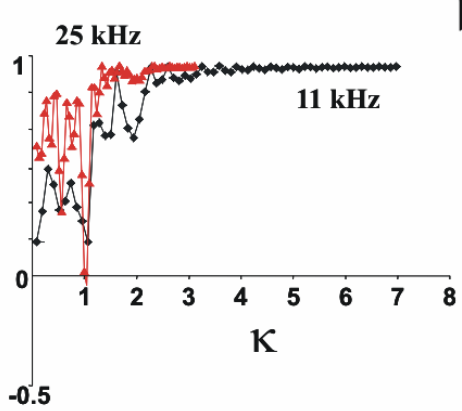

b)

c)

d)
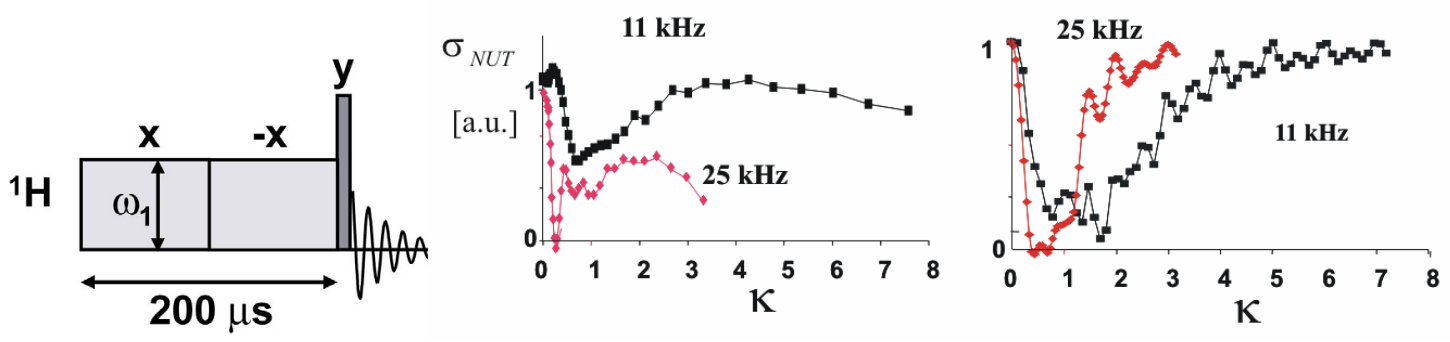

Figure 2.2. Spin-lock (a, b) and nutation (c, d) behavior of ${ }^{1} \mathrm{H}$ resonances in the tri-peptide AGG for the two indicated MAS rates. Experimental results (a, c) were obtained on a $600 \mathrm{MHz}$ wide-bore (Bruker Biospin, Germany) NMR instrument. Numerical simulations of the spin-lock (b) and nutation (d) behavior result from considering a dipolar coupled two-spin system for the two indicated MAS rates. All simulations were obtained using the $\mathrm{C}++$ based NMR simulation environment GAMMA ${ }^{58,63}$. A stepwise integration procedure (see, for example, $\mathrm{ref}^{64}$ ) was utilized to calculate the time evolution of the spin system. Results of 1000 single-crystal orientations were added. The total signal intensity after an evolution of $200 \mu \mathrm{s}$ is shown as a function of $\kappa=\omega_{1} / \omega_{R}$.

In addition to an efficient ${ }^{1} \mathrm{H}-{ }^{1} \mathrm{H}$ transfer, an ideal ${ }^{1} \mathrm{H}-{ }^{1} \mathrm{H}$ mixing sequence should exhibit a minimized signal loss of the total ${ }^{1} \mathrm{H}$ magnetization during the mixing time. Therefore, the relaxation behavior of ${ }^{1} \mathrm{H}$ signal intensities of unlabeled Ala-Gly-Gly under the conditions of radio frequency (r.f.) pulse spin-locking and nutation with phase inversion was investigated. Most mixing sequences can be characterized by one of these conditions. In Figure 2.2 signal intensities are plotted after an r.f. (spin-lock or nutation) evolution time of $200 \mu$ s as a function of the ratio of r.f. field and MAS rate $\kappa=\omega_{1} / \omega_{R}$. Here and in the following, experimental data points relate to single 
measurements and a statistical analysis of the error was thus omitted. At a spinning speed of $25 \mathrm{kHz}$, resonance minima around $\kappa \in[0.5,1,2]$ were observed in both experimental cases. This is in line with results obtained for rare-spins ${ }^{27,65-68}$. At a lower spinning speed of $11 \mathrm{kHz}$, the overall signal pattern is further complicated. For both MAS rates, spin locking is most efficient for the strongest applied r.f. fields (Figure 2.2a). Signal loss under r.f. nutation (Figure 2.2c) is minimal for very small or large r.f. fields. So as to ensure that the experimentally observed signal loss is indeed induced by strong dipolar ${ }^{1} \mathrm{H}-{ }^{1} \mathrm{H}$ couplings, full quantum mechanical simulations within the numerical simulation routine GAMMA $^{58,63}$ were carried out (see section 2.3). Because dipolar ${ }^{1} \mathrm{H}^{-1} \mathrm{H}$ interactions can be of comparable size to the applied MAS rate, a stepwise integration procedure (see e.g., ref ${ }^{64}$ ) of the relevant two-spin Hamiltonian was performed. In Figure 2.2, the spin-lock (b) and nutation (d) behavior predicted for the two MAS rates considered experimentally is shown. Again, the signal intensity was determined after $200 \mu$ s of spin system evolution as a function of $\kappa=\omega_{1} / \omega_{R}$. In agreement with the experimental results, favorable spin-lock and nutation behavior is detected for large ratios $\kappa=\omega_{1} / \omega_{R}$. Small values of $\kappa$ are also possible for nutation experiments. In general, the agreement between the theoretical predictions of a homonuclear dipolar coupled two-spin system and the empirical findings is better for ultrafast MAS conditions. A further improvement between simulation and experiment may be obtained by taking into account r.f. inhomogeneity and offset effects or by including additional spins in the QM calculation.

Three (nutation) r.f. schemes compatible with the theoretical and experimental results are summarized in Figure 2.3. Longitudinal mixing (LM, "Spin diffusion", Figure 2.3a) fulfills the experimental restrictions obtained from Figure 2.2 (small value of $\kappa)$. Moreover, polarization transfer schemes that are characterized by relatively high values of $\kappa$ minimize relaxation effects. For example, SEDRA $^{69} /$ RFDR $^{70}$ schemes that involve strong, rotor-synchronized $\pi$ pulses are known to promote homonuclear dipolar recoupling among ${ }^{13} \mathrm{C}$ nuclei ${ }^{69,70}$. In Figure $2.3 \mathrm{~b}$, the corresponding RFDR implementation of a ${ }^{1} \mathrm{H}-{ }^{1} \mathrm{H}$ mixing experiment is shown. Both $\mathrm{LM}$ and RFDR represent laboratory frame mixing schemes and are, as demonstrated below, characterized by zero-quantum polarization transfer. Finally, rotating frame double- 
quantum schemes that are characterized by large values of $\kappa$ can be utilized. In Figure $2.3 \mathrm{c}$, the POST-C7 scheme as published by Hohwy et al. ${ }^{71}$ has been adapted.

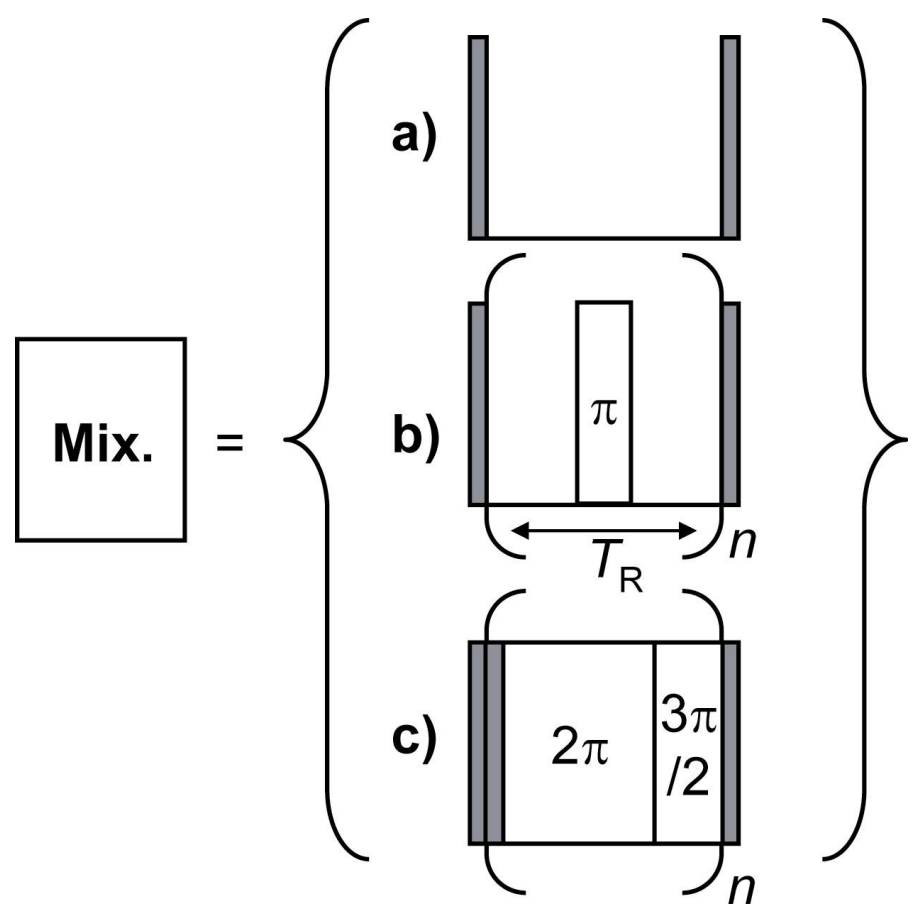

Figure 2.3. ${ }^{1} \mathrm{H}-{ }^{1} \mathrm{H}$ mixing schemes considered in the current study. (a) relates to longitudinal mixing (LM), (b) to RFDR ${ }^{70}$ zero-quantum (0Q) mixing and (c) to POST-C7 ${ }^{71}$ double-quantum (2Q) mixing. Shaded pulses correspond to $90^{\circ}$ pulses. In (b) and (c), $n$ refers to the number of rotor periods $T_{\mathrm{R}}$ and basic POST-C7 cycles, respectively.

\subsection{Quantum-mechanical simulations}

For the quantum-mechanical two-spin simulations shown in Figure 2.2b and $\mathrm{d}$ and in Figure 2.9, a dipolar coupled $\mathrm{I}_{2}$ spin system is considered. Neglecting scalar throughbond couplings, the relevant internal system Hamiltonian in the Zeeman interaction frame (laboratory frame, LAB) contains dipolar and chemical shielding interactions:

$$
\begin{aligned}
& H_{C S A}(t)=A_{00}^{C S A} T_{00}^{C S A}+A_{20}^{C S A}(t) T_{20}^{C S A} \\
& H_{D}(t)=A_{20}^{D}(t) T_{20}^{D}
\end{aligned}
$$

In equation 2.3, the Hamilton operators of chemical shielding anisotropy (CSA) and dipolar coupling interactions are expressed as products of spherical space $\left(A_{\mathrm{kq}}\right)$ and spin $\left(T_{\mathrm{kq}}\right)$ tensor components ${ }^{61}$. The explicit time dependence in equation 2.3 stems 
from the modulation of the rank $\mathrm{k}=2$ spherical (dipolar and CSA) space tensors under MAS. The spin tensor components are not influenced by MAS:

$$
\begin{aligned}
& T_{00}^{C S A}=-\frac{1}{\sqrt{3}} I_{z} B_{0} \\
& T_{20}^{C S A}=\sqrt{\frac{2}{3}} I_{z} B_{0} \\
& T_{20}^{D}=\frac{1}{\sqrt{6}}\left(3 I_{1 z} I_{2 z}-\vec{I}_{1} \cdot \vec{I}_{2}\right)
\end{aligned}
$$

The space tensor components in the principal axis system (PAS) of the interaction tensors depend on tensor anisotropy $\delta$, asymmetry $\eta$, and the isotropic part $a$ :

$$
\begin{aligned}
& A_{00}^{(P A S)}=-\frac{1}{\sqrt{3}} a \\
& A_{20}^{(P A S)}=\sqrt{\frac{3}{2}} \delta \\
& A_{2 \pm 2}^{(P A S)}=\frac{1}{2} \delta \cdot \eta
\end{aligned}
$$

In the case of CSA (assuming $\sigma_{33} \geq \sigma_{22} \geq \sigma_{11}$ ):

$$
\begin{aligned}
& a=\operatorname{Tr}\left(\begin{array}{ccc}
\sigma_{11} & 0 & 0 \\
0 & \sigma_{22} & 0 \\
0 & 0 & \sigma_{33}
\end{array}\right) \\
& \delta=\sigma_{33}-a / 3 \\
& \eta=\frac{\sigma_{22}-\sigma_{11}}{\delta}
\end{aligned}
$$

For the dipolar coupling one finds:

$$
\begin{aligned}
& a=0 \\
& \delta=-2 \frac{\mu_{0} \gamma_{i} \gamma_{j} \hbar}{4 \pi r_{i j}^{3}} \\
& \eta=0
\end{aligned}
$$


The space tensor components in the PAS are related to elements in a rotor-fixed axis system by an Euler transformation $(\alpha, \beta, \gamma)$. Subsequently the components are transformed to the laboratory frame by a second Euler transformation $\left(\omega_{R} t, \theta_{M}, 0\right)$ :

$$
\begin{aligned}
& A_{l m}^{(R A S)}=\sum_{m^{\prime}=-l}^{l} A_{l m^{\prime}}^{(P A S)} e^{-i \alpha m^{\prime}} d_{m^{\prime} m}^{l}(\beta) e^{-i \gamma m} \\
& A_{l m}^{(L A B)}(t)=\sum_{m^{\prime}=-l}^{l} A_{l m^{\prime}}^{(R A S)} e^{-i \omega_{R} t m^{\prime}} d_{m^{\prime} m}^{l}\left(\theta_{M}\right)
\end{aligned}
$$

$d$ represents the reduced Wigner element ${ }^{72}$ and $\theta_{M}$ and $\omega_{R}$ denote the magic angle and the MAS rate, respectively. Assuming a time-independent r.f. field:

$$
H_{r f}=\omega_{1}\left(I_{1 x}+I_{2 x}\right)
$$

nutation (NUT) and spin-lock (SL) signals calculated in Figure 2.2b and $\mathrm{d}$ are determined by the initial density operators:

$$
\sigma_{S L}(t=0)=I_{1 x}+I_{2 x} \quad \sigma_{N U T}(t=0)=I_{1 z}+I_{2 z}
$$

The density matrix $\sigma(t)$ evolves under the time-dependent Hamiltonian $H(t)=H_{C S A}(t)+H_{D}(t)+H_{r f}$ according to the Liouville-von-Neuman equation:

$$
\frac{d}{d t} \sigma(t)=-i[H(t), \sigma(t)]
$$

The signal intensity plotted in Figure 2.2 is subsequently obtained from a step-wise integration procedure (see e.g. ref $^{64}$ ) of equation 2.11 within the numerical simulation routine GAMMA ${ }^{58,63}$.

For the two-spin calculation shown as dashed lines in Figure 2.9, equation 2.9 was replaced by a time-dependent r.f. contribution that reflects the details of the POST-C7 recoupling sequence. The cross-peak intensity was here obtained by assuming an initial density operator $I_{1 z}$ and a detection operator $I_{2 z}$. Similar numerical simulations 
were conducted for the case of LM (Figure 2.5, see Appendix B.1) and RFDR mixing (Figure 2.7) assuming standard literature values ${ }^{59-61}$ for the isotropic and anisotropic ${ }^{1} \mathrm{H}$ chemical shielding parameters.

\subsection{Molecular-dynamics based structure calculations}

All structure calculations were performed within $\mathrm{CNS}^{73}$ (Crystallography and NMR System). Calculations implemented the PROTEIN-ALLHDG ${ }^{74}$ parameter file. ${ }^{1} \mathrm{H}-{ }^{1} \mathrm{H}$ distance constraints obtained from CHHC / NHHC 2D spectra were treated as NOE constraints represented by a square-well potential:

$$
E_{S S N M R}=\sum k_{H H}\left\{\begin{array}{lc}
\left(r_{i j}-r_{i j}^{u}\right)^{2} & r_{i j}>r_{i j}^{u} \\
0 & r_{i j}^{l} \leq r_{i j} \leq r_{i j}^{u} \\
\left(r_{i j}-r_{i j}^{l}\right)^{2} & r_{i j}<r_{i j}^{l}
\end{array}\right.
$$

where $r_{\mathrm{ij}}$ is the calculated distance between protons $\mathrm{i}$ and $\mathrm{j}, r_{\mathrm{ij}}{ }^{l}$ and $r_{\mathrm{ij}}{ }^{u}$ are the values of the lower and upper limits of the target distances, respectively, and $k_{\mathrm{HH}}$ is the force constant. In case of ambiguous ${ }^{1} \mathrm{H}-{ }^{1} \mathrm{H}$ constraints involving methyl or methylene protons, $r_{\mathrm{ij}}$ is computed by an $r^{-6}$ summation $^{75}$ involving all possible proton-proton contacts. Extended conformers of Ala-Gly-Gly and L-Histidine. $\mathrm{HCl}$ were created as initial structures and subsequently subjected to a simulated annealing protocol consisting of three stages:

L-Histidine $\cdot \mathrm{HCl}$. 1. High-temperature annealing in torsion angle space, in 2000 time steps of $0.002 \mathrm{ps}$ at $30000 \mathrm{~K}$. 2. Slow-cool annealing stage in torsion angle space, in 6000 steps of $0.002 \mathrm{ps}$, and temperature reduction from $30000 \mathrm{~K}$ to zero in steps of 250 K. 3. Final conjugate gradient minimization of 3000 steps. During all stages, a force constant $k_{\mathrm{HH}}$ of $150 \mathrm{kcal} \mathrm{mol}^{-1} \AA^{-2}$ was used. A set of 100 conformers was generated, starting from different initial velocities. The resulting structures were sorted by total energy. 
Ala-Gly-Gly. 1. High-temperature annealing in torsion angle space, in 2000 time steps of 0.005 ps at $50000 \mathrm{~K}$. 2. Slow-cool annealing stage in torsion angle space, in 4000 steps of $0.005 \mathrm{ps}$, and temperature reduction from $50000 \mathrm{~K}$ to zero in steps of $250 \mathrm{~K}$. 3. Final conjugate gradient minimization of 2000 steps. Force constants $k_{\mathrm{HH}}$ of 150 kcal mol ${ }^{-1} \AA^{-2}$ were employed during high-temperature and slow-cool annealing, and $75 \mathrm{kcal} \mathrm{mol}^{-1} \AA^{-2}$ during final minimization. Dihedral angles derived using TALOS $^{76}$ were restrained by harmonic potentials analogous to equation 2.12 with force constants $k$ of 100,200 , and $400 \mathrm{kcal} \mathrm{mol}^{-1} \mathrm{rad}^{-2}$ during the three stages, respectively. The RMSDs given by TALOS for each backbone angle were taken as allowed rotations around the predicted angles without energy contribution. A set of 50 conformers was generated, starting from different initial velocities.

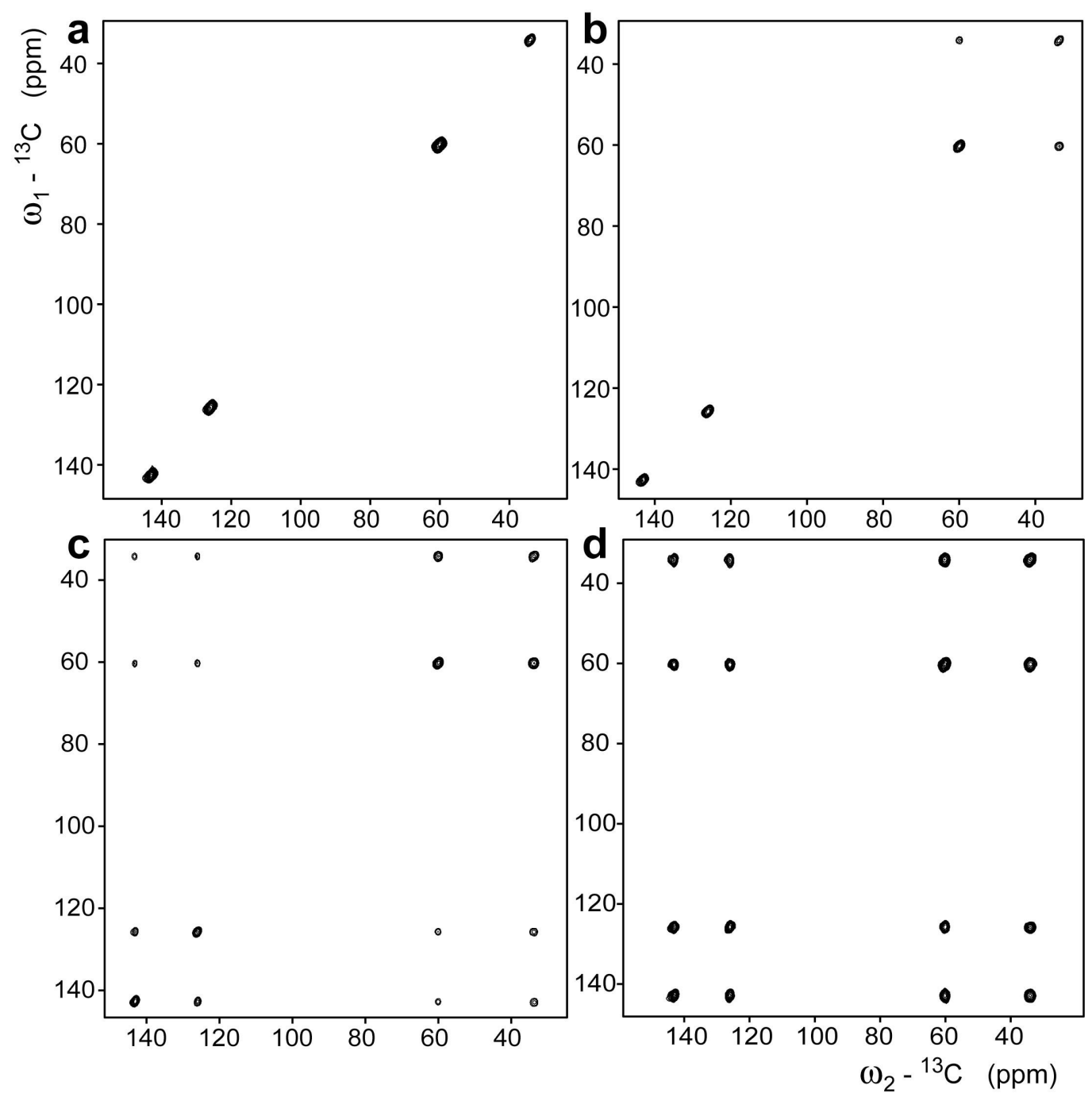

Figure 2.4. Experimentally observed CHHC cross-peak intensities for Histidine $\cdot \mathrm{HCl}$ at $11 \mathrm{kHz}$ MAS on a $400 \mathrm{MHz}$ wide-bore NMR instrument for RFDR mixing (Figure 2.3b) employing four different mixing times: (a) $0 \mu \mathrm{s}$, (b) $182 \mu \mathrm{s}$, (c) $273 \mu \mathrm{s}$, and (d) $364 \mu \mathrm{s}$. Cross-peak intensities reflect ${ }^{1} \mathrm{H}-{ }^{1} \mathrm{H}$ interactions, encoded in ${ }^{13} \mathrm{C}$ evolution and detection periods. 


\subsection{Analysis of zero-quantum ${ }^{1} \mathrm{H}-{ }^{1} \mathrm{H}$ polarization transfer}

To introduce the general concept of a CHHC 2D correlation experiment, Figure 2.4 contains results of a $0 \mathrm{Q}-\mathrm{CHHC}$ (utilizing the RFDR mixing scheme of Figure 2.3b) on uniformly $\left[{ }^{13} \mathrm{C},{ }^{15} \mathrm{~N}\right]$ labeled Histidine $\cdot \mathrm{HCl}$ at $11 \mathrm{kHz} \mathrm{MAS}$ on a $400 \mathrm{MHz}$ instrument. $\mathrm{CP}$ times before and after proton mixing were optimized for polarization transfer within $\mathrm{CH}_{x}(x=1-3)$ groups only. The NMR signal of the non-protonated ring carbon $\mathrm{C} \gamma$ is correspondingly missing. As expected, no cross-peak intensities for vanishing mixing times (Figure 2.4a) are observed. Increasing the mixing times from $182 \mu \mathrm{s}$ (Figure 2.4b) to $364 \mu \mathrm{s}$ (Figure $2.4 \mathrm{~d}$ ) gradually establishes all ${ }^{13} \mathrm{C}-{ }^{13} \mathrm{C}$ correlations in the side chain spectrum. Because no ${ }^{1} \mathrm{H}$ evolution or detection periods are present, the $\mathrm{CHHC} / \mathrm{NHHC}$ concept does not allow for the identification of individual ${ }^{1} \mathrm{H}-{ }^{1} \mathrm{H}$ contacts, for example involving $\mathrm{CH}_{2}$ or $\mathrm{CH}_{3}$ groups. Hence, in the following, reference will be made only to ${ }^{13} \mathrm{C}-{ }^{13} \mathrm{C}$ correlations and it will be tacitly assumed that they are mediated by next neighbor proton-proton interactions.

For a further analysis, Figure 2.5 shows experimentally observed CHHC cross-peak intensities for a larger set of (LM) transfer times for an MAS rate of $11 \mathrm{kHz}$ (a) and $26 \mathrm{kHz}$ (b). Here, and in all results presented in the following, the cross-peak intensities were normalized against the total intensity of the spectrum. Experimental data points are shown for the pairs $\mathrm{C} \alpha-\mathrm{C} \beta$ (filled squares) and $\mathrm{C} \beta-\mathrm{C} \delta$ (filled diamonds). From neutron diffraction data ${ }^{77},{ }^{1} \mathrm{H}-{ }^{1} \mathrm{H}$ distances in Histidine $\cdot \mathrm{HCl}$ are predicted to be $2.22 \AA$ for the $C \alpha-C \beta$ pair and $3.06 \AA$ for the nearest neighbor $\mathrm{C} \beta-\mathrm{C} \delta$ pair. A simple inspection of Figure 2.5 reveals that the shorter ${ }^{1} \mathrm{H}-{ }^{1} \mathrm{H}$ distance (i.e., the $\mathrm{C} \alpha-\mathrm{C} \beta$ pair) is characterized by a significantly faster buildup and, correspondingly, a stronger transfer efficiency in the initial rate regime $t_{\mathrm{HH}}=[0,200 \mu \mathrm{s}]$ in Figure 2.5a and $t_{\mathrm{HH}}=[0,1000 \mu \mathrm{s}]$ in Figure 2.5b. Attempts to describe the experimentally detected transfer dynamics within a dipolar coupled two-spin simulation (see Appendix B.1) were unsuccessful. This indicates that the transfer dynamics result from an extended network of dipolar coupled spins. Instead, theoretical results shown in Figure 2.5a and $\mathrm{b}$ were obtained using the relaxation analysis of equation 2.1. Because the ${ }^{1} \mathrm{H}-{ }^{1} \mathrm{H}$ distances are known, the value of the zero-quantum (0Q) lineshape function can be determined from the experimentally observed transfer rates. For 
the data recorded at $11 \mathrm{kHz}$ MAS (Figure 2.5a), a value $J^{0 Q, L M}(\Delta)=208 \mu \mathrm{s}$ is obtained. In agreement with a relaxation-based theoretical model, the signal buildup is exponential and the zero-quantum spin diffusion rate under LM mixing scales inversely with the $6^{\text {th }}$ power of the inter-nuclear ${ }^{1} \mathrm{H}-{ }^{1} \mathrm{H}$ distance.

In Figure $2.5 \mathrm{~b}$, the experiment was repeated at an MAS rate of $26 \mathrm{kHz}$. For both considered proton-proton contacts, the transfer rates, and hence, the 0Q line-shape functions are significantly reduced. Although a direct determination of the 0Q line shape function is difficult, it can be approximated by a product ${ }^{78}$ of two 1Q decay functions known to scale with $1 / \omega_{r}{ }^{79,80}$. Thus, the $0 \mathrm{Q}$-line shape function should scale with $1 / \omega_{r}^{2}$. Indeed, a value of $J^{0 Q, L M}(\Delta)=37 \mu$ s leads to very good agreement between theory and experimental data for the shorter distance (Figure 2.5b) and reasonable agreement for the $\mathrm{C} \beta-\mathrm{C} \delta$ pair. In light of these experimental observations, the assumption of a uniform diffusion constant that is not influenced by the details of the experiment is certainly not justified.
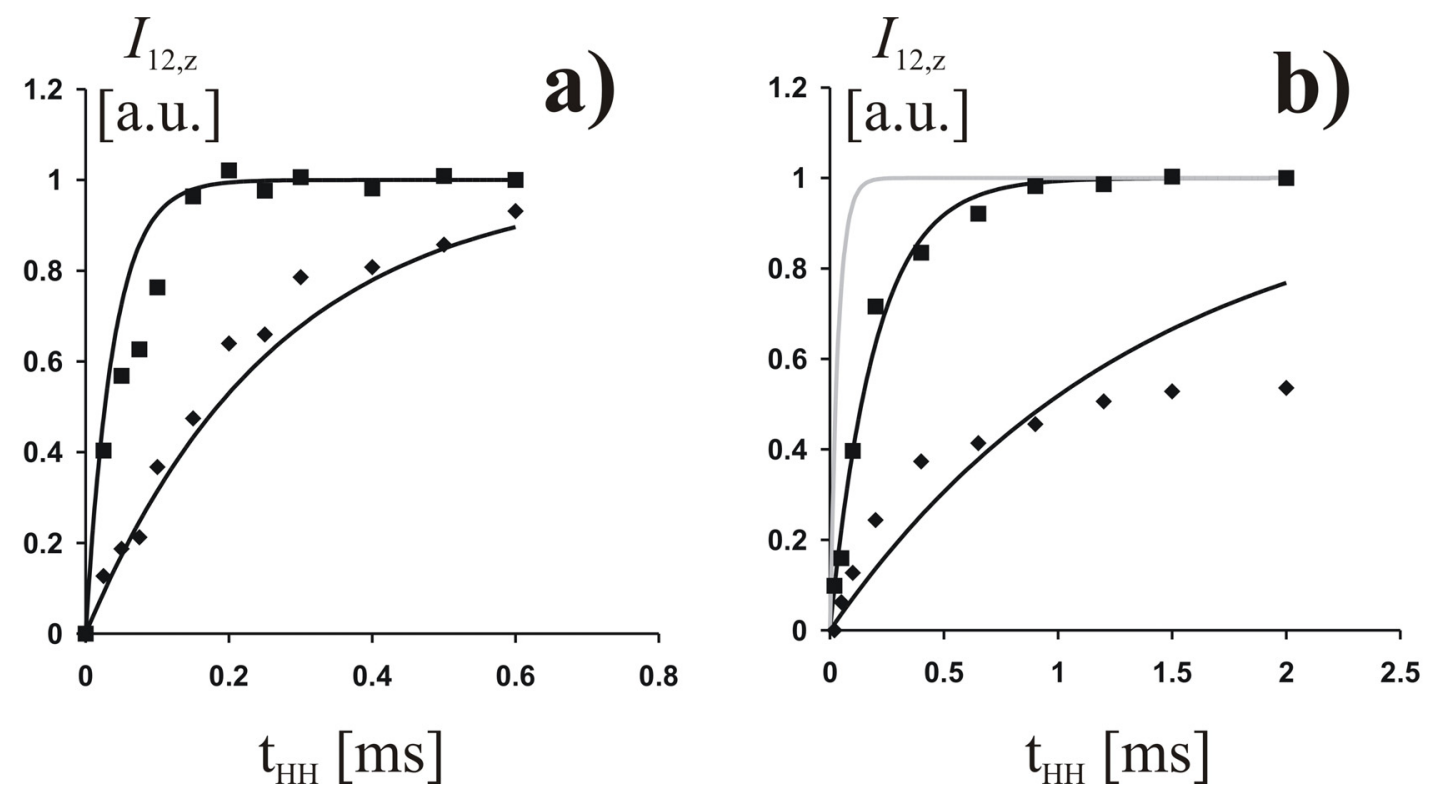

Figure 2.5. Polarization transfer rates under the LM scheme on $\mathrm{U}-\left[{ }^{13} \mathrm{C},{ }^{15} \mathrm{~N}\right]$ labeled Histidine $\cdot \mathrm{HCl}$ observed at an MAS rate of $11 \mathrm{kHz}$ (a) and $26 \mathrm{kHz}$ (b). Data sets were calibrated as follows: The observed cross-peak intensities for the $\mathrm{C} \alpha-\mathrm{C} \beta$ (filled squares) and $\mathrm{C} \beta-\mathrm{C} \delta$ (filled diamonds) were normalized against the total intensity of the $2 \mathrm{D}$ spectrum. Subsequently, data points for the fastest buildup were set to 1 for long mixing times. Indicated lines correspond to numerical simulations using equation 2.1, the $0 \mathrm{Q}$ line-shape function given in the text and the following simulation parameters: $r_{12}$ $=2.22 \AA\left(\mathrm{C} \alpha-\mathrm{C} \beta\right.$ spin pair) and $r_{12}=3.06 \AA\left(\mathrm{C} \beta-\mathrm{C} \delta\right.$ spin pair). The signal buildup reflects ${ }^{1} \mathrm{H}-{ }^{1} \mathrm{H}$ interactions, encoded in ${ }^{13} \mathrm{C}$ evolution and detection periods. For reference, predictions using $J^{0 \mathrm{Q}, \mathrm{LM}}(\Delta)$ obtained at $11 \mathrm{kHz}$ are included in gray (b). 
Next, the polarization transfer behavior for different static magnetic fields was investigated. In Figure 2.6, correlations recorded at $11 \mathrm{kHz}$ MAS and (a) $9.4 \mathrm{~T}$ are compared to data obtained at (b) $14.1 \mathrm{~T}$. Again, uniformly $\left[{ }^{13} \mathrm{C},{ }^{15} \mathrm{~N}\right]$ labeled Histidine $\cdot \mathrm{HCl}$ was considered. Apart from the $\mathrm{C} \alpha-\mathrm{C} \beta$ and $\mathrm{C} \delta-\mathrm{C} \varepsilon$ connectivities, all correlations, including the $3.06 \AA(\mathrm{C} \beta-\mathrm{C} \delta)$ contact, are characterized by slower buildup rates that increase in intensity beyond the mixing times considered. The relatively fast signal buildup of the $\mathrm{C} \delta-\mathrm{C} \varepsilon$ pair in Figure 2.6a most likely results from relay transfers involving ${ }^{15} \mathrm{~N}$ bound ${ }^{1} \mathrm{H}$ spins in the aromatic ring (see also Figure 2.10).

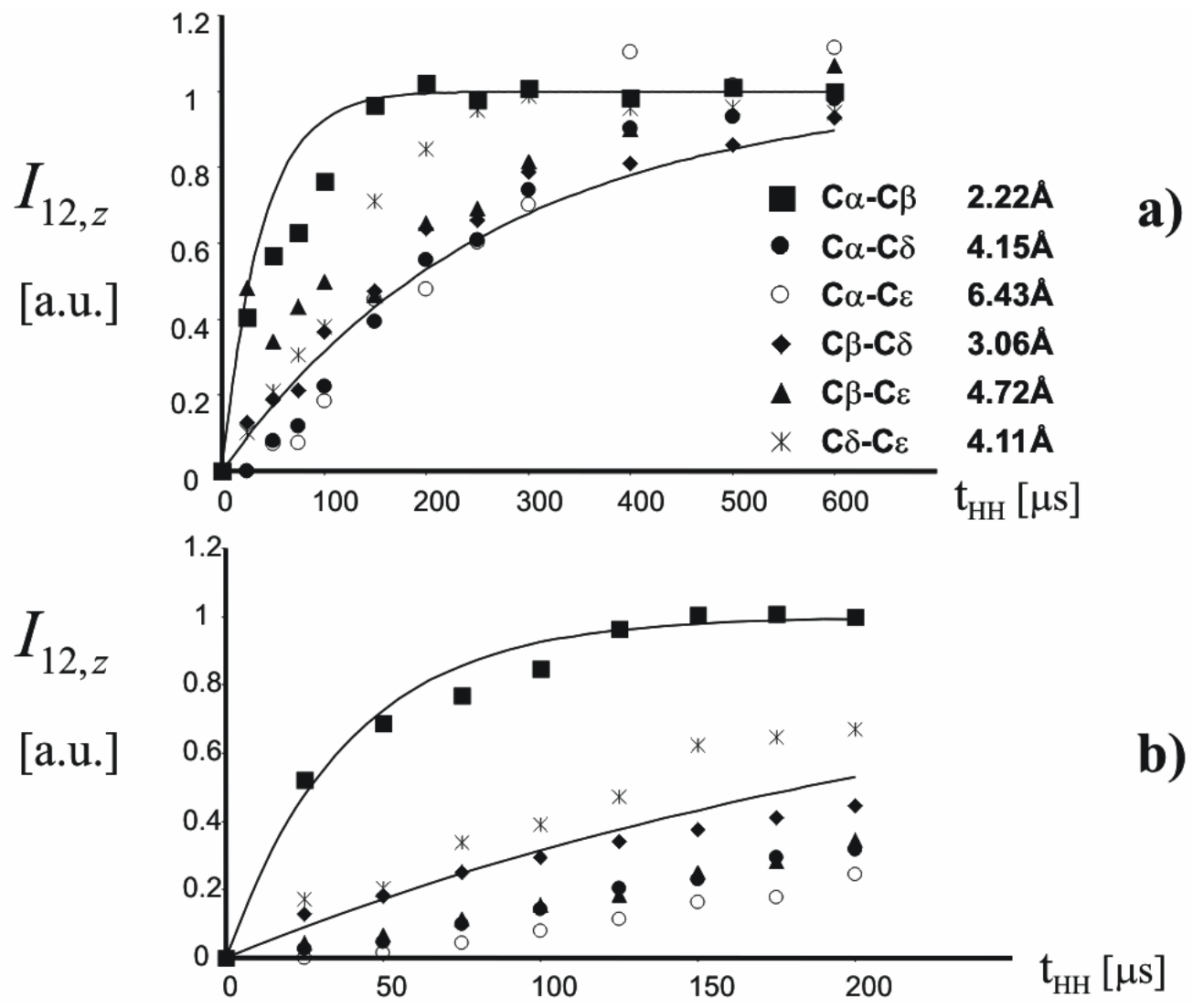

Figure 2.6. Experimentally observed polarization transfer rates on $\mathrm{U}-\left[{ }^{13} \mathrm{C},{ }^{15} \mathrm{~N}\right]$ labeled Histidine $\cdot \mathrm{HCl}$ at (a) $400 \mathrm{MHz}$ and (b) $600 \mathrm{MHz}$ under LM mixing. Indicated distances were obtained from previous neutron diffraction studies ${ }^{77}$. Lines correspond to numerical simulations using equation 2.1 and the following simulation parameters: (a): $r_{12}=2.22 \AA$ (C $\alpha$-C $\beta$ spin pair), $r_{12}=3.06 \AA$ (C $\beta$ - $C \delta$ spin pair) and the $0 \mathrm{Q}-$ line shape function given in the text. Note that in (b) the time axis is expanded by a factor 3. The signal buildup reflects ${ }^{1} \mathrm{H}^{-1} \mathrm{H}$ interactions, encoded in ${ }^{13} \mathrm{C}$ evolution and detection periods.

Remarkably, the theoretical curves (included from Figure 2.5a) largely describe the buildup characteristics for both 400 and $600 \mathrm{MHz}$ NMR data, indicating that the dominating transfer mechanism for proton-proton distances up to $3 \AA$ is only weakly 
influenced by an increase in isotropic and anisotropic ${ }^{1} \mathrm{H}$ chemical shielding interactions. Apart from a reduction in transfer rates for long distances, the data at higher field strength reveal that the transfer curves do not allow for discrimination between direct contacts and relay mechanisms for distances larger than $3 \AA$. Within experimental error, these buildup rates behave similarly to the $3.06 \AA$ distance predicted for the $\mathrm{C} \beta-\mathrm{C} \delta$ pair. As in the solution-state, these curves most likely involve relay transfer mechanisms and hence complicate a discrimination between a $3.06 \AA$ ${ }^{1} \mathrm{H}-{ }^{1} \mathrm{H}$ direct contact and multiple-spin transfer mechanism over a comparable length scale. The experimental results obtained using the LM scheme of Figure 2.3a are therefore in qualitative agreement with the theoretical description.

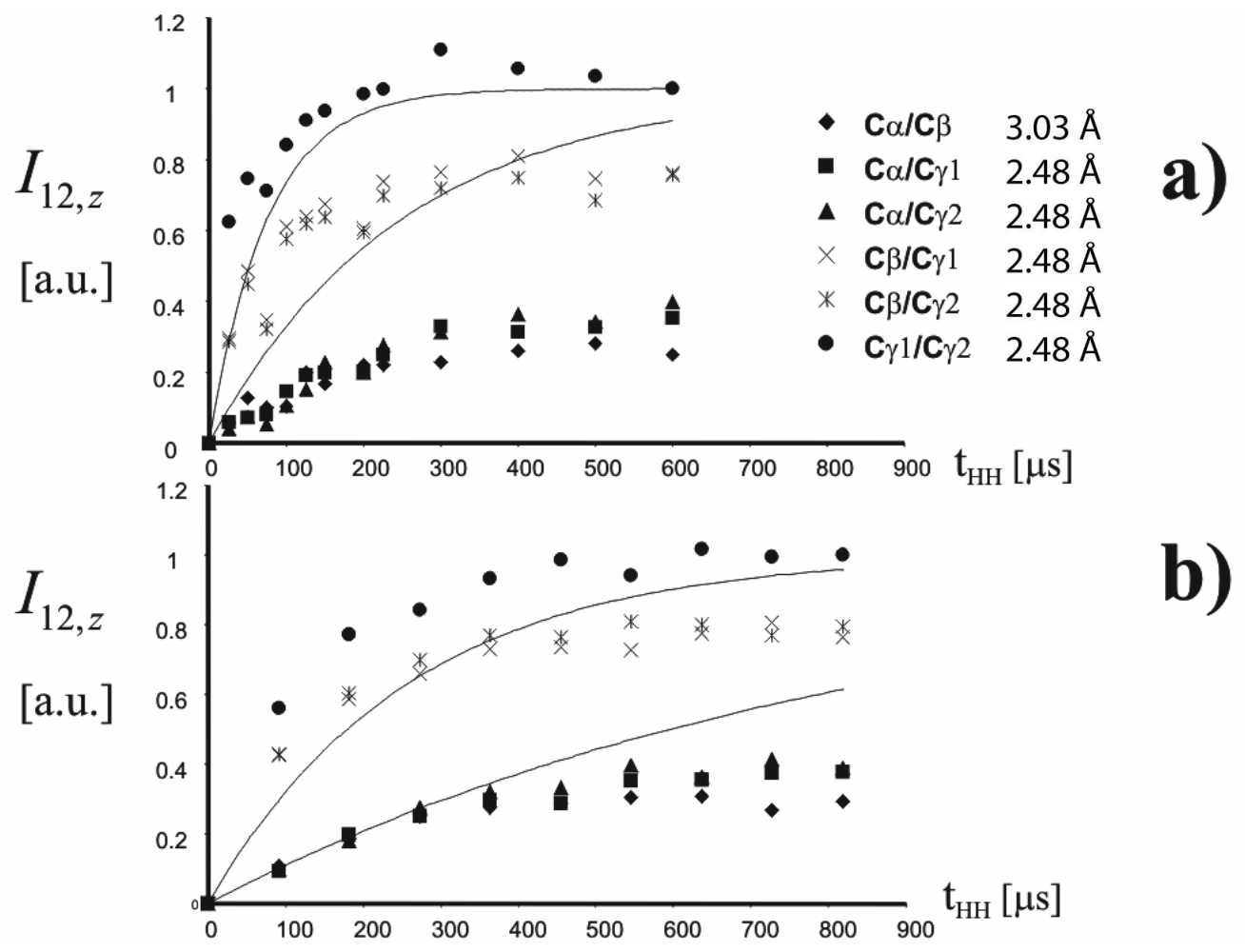

Figure 2.7. $0 \mathrm{Q}-\mathrm{CHHC}$ cross-peak buildups in $\mathrm{U}-\left[{ }^{13} \mathrm{C},{ }^{15} \mathrm{~N}\right]$ labeled L-Valine for longitudinal (a) and RFDR (b) ${ }^{1} \mathrm{H}-{ }^{1} \mathrm{H}$ mixing. Spin pairs are indicated by symbols. Lines correspond to theoretical predictions using the 0Q line-shape function of Figures 2.5 and 2.6 and ${ }^{1} \mathrm{H}-{ }^{1} \mathrm{H}$ distances of $2.48 \AA$ and $3.03 \AA$ A. Data were obtained on a wide-bore $400 \mathrm{MHz}$ instrument. The signal buildup reflects ${ }^{1} \mathrm{H}-{ }^{1} \mathrm{H}$ interactions, encoded in ${ }^{13} \mathrm{C}$ evolution and detection periods.

To investigate whether these observations also hold for NMR data obtained in other systems, Figure 2.7 a contains experimental results on $U-\left[{ }^{13} \mathrm{C},{ }^{15} \mathrm{~N}\right]$ labeled L-Valine obtained under longitudinal mixing (LM) at $11 \mathrm{kHz}$ MAS. According to the crystal 
structure ${ }^{81}$, all nearest neighbor ${ }^{1} \mathrm{H}-{ }^{1} \mathrm{H}$ distances are, except for the $\mathrm{C} \alpha-\mathrm{C} \beta$ pair $(3.03$ $\AA$ ), identical and short (2.48 $\AA$ ). Transfer functions assuming the same 0Q line-shape function as in Figure 2.5a are included for inter-nuclear distances of $2.48 \AA$ and 3.03 $\AA$ as reference. An inspection of Figure 2.7 leads to the following conclusions: (i) the overall intensity of the different spin pairs varies considerably. Because the crosspeak intensities were (as in Figures 2.5 and 2.6) normalized against the total intensity of the spectrum, this observation can be partially attributed to intensity variations due to mobility effects. For example, cross-peak amplitudes involving one or two $\mathrm{CH}_{3}$ groups are in general stronger (Figure 2.7). (ii) One in general finds cross-peak maxima between 300 and $500 \mu$ s consistent with proton-proton distances of about 2.4 $\AA$. Correspondingly, a further downscaling of the theoretical curve for the $2.48 \AA$ distance leads to good agreement for the majority of the considered spin pairs. It should be noted that an additional normalization is only justified for buildup curves that display a clear maximum in the considered mixing time regime. (iii) The experimental data do not allow for a reliable detection of the $\mathrm{C} \alpha-\mathrm{C} \beta$ distance of 3.03 $\AA$. This effect can be attributed to strong relay mechanisms within the highly symmetric arrangement of L-Valine that influence the transfer dynamics for the C $\alpha$ $\mathrm{C} \beta$ pair. With the exception of this discrepancy, the experimentally observed transfer rates are hence in qualitative agreement with distances obtained from X-ray crystallography. In the solution-state, ${ }^{1} \mathrm{H}-{ }^{1} \mathrm{H}$ contacts involving methyl protons are usually described by modified NOE buildup rates ${ }^{82-85}$. Although analogous effects in the model systems considered here were not observed, internal motions could further influence the observed transfer characteristics. Examples involving mobile polymer systems have been reported ${ }^{86}$.

To further investigate the dependence of the transfer dynamics on experimental settings, results (Figure 2.7b) obtained using RFDR as a ${ }^{1} \mathrm{H}-{ }^{1} \mathrm{H}$ mixing sequence (Figure 2.3b) were included. Again, the correlation spectra are dominated for short mixing times by the strongest ${ }^{1} \mathrm{H}-{ }^{1} \mathrm{H}$ interactions. RFDR transfer leads, in zeroth order average Hamiltonian theory, to a dipolar Hamiltonian ${ }^{70}$ with a recoupling efficiency given by the product of the dipolar coupling element and the (isotropic and anisotropic) ${ }^{1} \mathrm{H}$ chemical shift difference (normalized against the MAS rate) of the two spins of interest. Correspondingly, numerical 2-spin simulations predict (for a 
proton-proton distance of $2.5 \AA$ ) a mixing time dependence that compares favorably to results obtained for one-bond ${ }^{13} \mathrm{C}-{ }^{13} \mathrm{C}$ dipolar recoupling experiments. Instead, the experimental buildup rates shown in Figure $2.7 \mathrm{~b}$ are significantly faster and are well described within a monoexponential 0Q-relaxation analysis (equation 2.1). In agreement with all other correlations, one observes an approximately 3 -fold reduction in the polarization transfer rate for the $\mathrm{C} \gamma 1-\mathrm{C} \gamma 2$ spin pair (filled circles in Figure 2.7). For the experimental parameters given in Figure 2.7, one hence finds a modified zeroquantum line-shape function. $J^{0 Q, R F D R}(\Delta) \cong 0.3 J^{0 Q, L M}(\Delta)$ favoring the LM approach in practical applications.

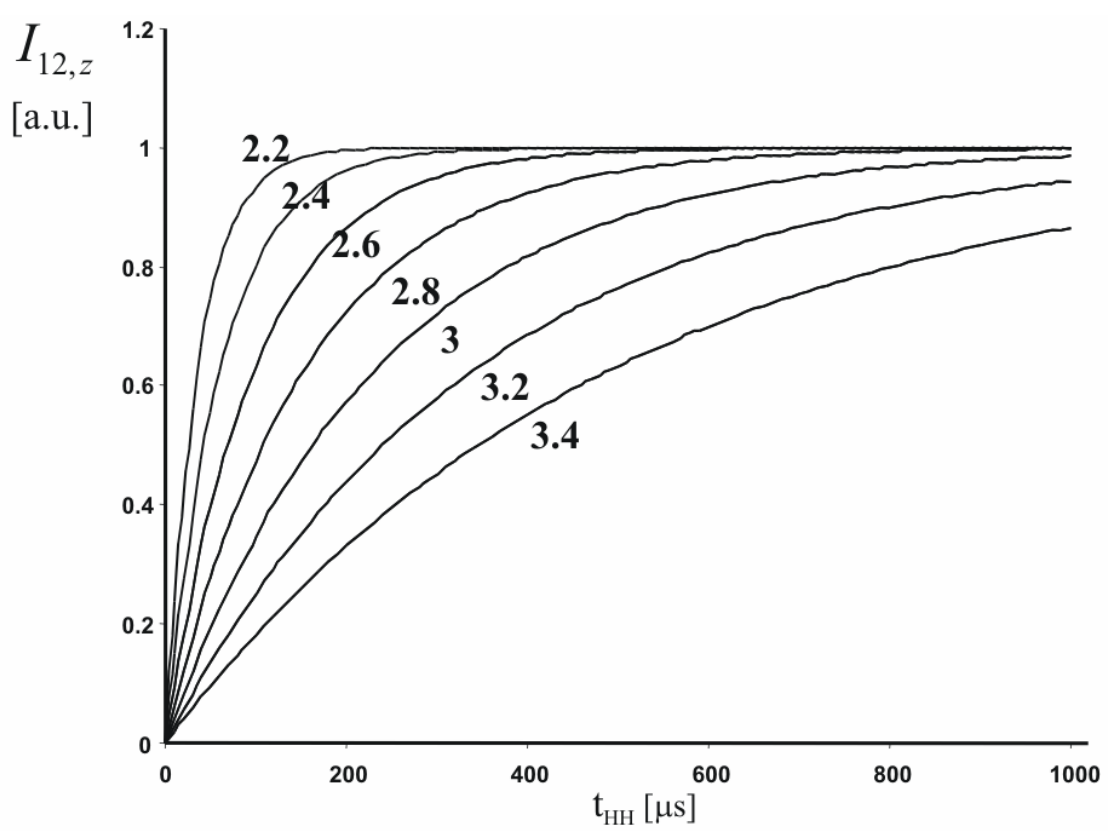

Figure 2.8. Simplified model describing the initial rate transfer behavior of the OQ-CHHC LM method. Transfer rates were selected according to the empirical results obtained in Figures 2.6 and 2.7. Assuming the validity of equation 2.1 , these parameters directly correlate with the ${ }^{1} \mathrm{H}-{ }^{1} \mathrm{H}$ distances given in $\AA$. The calibration is valid for experiments at 400 and $600 \mathrm{MHz}$.

The presented results of Figures 2.5-2.7 suggest a general approach for the structural analysis of 0Q-CHHC correlation spectra in systems of unknown structure: A semiclassical relaxation treatment leads to a uniform zero-quantum line-shape function $J^{0 Q}(\Delta)$ for a given MAS rate, $0 \mathrm{Q}$ mixing scheme and $\mathrm{B}_{0}$ field. As exemplified in Figure 2.8 for the LM scheme and an MAS rate of $11 \mathrm{kHz}$, one can subsequently construct a transfer map that scales inversely with the $6^{\text {th }}$ power of the inter-nuclear distance. The most reliable structural analysis involves a detailed 
comparison of the theoretical predictions to the experimentally detected cross-peak buildup intensities. As visible from Figures 2.5-2.8, such an analysis permits to detect short ${ }^{1} \mathrm{H}-{ }^{1} \mathrm{H}$ distances with relatively high accuracy $( \pm 0.2 \AA)$. Alternatively, one can solely rely on results obtained for one mixing time in the initial rate regime where the observed signal intensities strongly correlate with the expected proton-proton distance. Similar to the solution-state, additional errors due to variations in signal intensity and relay transfer effects must then be taken into account.

One can compare the results regarding 0Q-CHHC mixing to a cross-relaxation analysis employed in solution-state NMR. Here, ${ }^{1} \mathrm{H}$ relaxation in the laboratory frame is dominated by dipolar ${ }^{1} \mathrm{H}-{ }^{1} \mathrm{H}$ interactions leading to nuclear Overhauser effects (NOE), and the rate of polarization transfer between two spins is determined by the Solomon ${ }^{40}$ equations. The cross-relaxation rate directly depends on the inter-nuclear distance between the two dipolar interacting spins under study and the details of the motional process ${ }^{39,41}$ encoded in the generalized spectral density functions $\tilde{J}\left(\omega_{1}, \omega_{2}\right)$ :

$$
\frac{1}{T^{N O E}}=\left(\mu_{0} / 4 \pi\right)^{2} \frac{\hbar^{2} \gamma_{I}^{4}}{r_{12}^{6}} \widetilde{J}\left(\omega_{1}, \omega_{2}\right)
$$

Thus, both solid and solution-state rate constants are proportional to $1 / r_{12}{ }^{6}$. Comparison of equation 2.13 and equation 2.2 furthermore reveals that the polarization transfer rates in the solid- and liquid-state are determined by multiplespin and motional effects within a dipolar coupled proton spin network, respectively. As in the solution-state, where a significant amount of information is available today regarding the spectral density functions $\tilde{J}\left(\omega_{1}, \omega_{2}\right)$ for different motional models ${ }^{85,87-89}$, the presented $\mathrm{CHHC}$ / NHHC concept hence allows for a further detailed analysis of the $0 \mathrm{Q}$ line shape function under variable experimental conditions and different degrees of proton density and molecular mobility. For the solid-phase systems considered here, the observed rate constants are about 3 orders of magnitude faster than observed in the solution-state. Additional complications due to scalar couplings ${ }^{42}$ can therefore safely be neglected. 


\subsection{Analysis of double-quantum ${ }^{1} \mathrm{H}-{ }^{1} \mathrm{H}$ polarization transfer}

As already demonstrated in ref ${ }^{53}$, it is also possible to establish double-quantum polarization transfer in fully protonated systems with transfer characteristics wellknown from rare-spin 2Q-correlation experiments. Here, a 0Q-spin diffusion approximation does not adequately describe the experimental findings. Instead, QM two-spin simulations were conducted for variable MAS rates indicating that POST-C7 dipolar recoupling is possible at $11 \mathrm{kHz}$. According to these calculations, increasing the MAS rate does not alter the transfer dynamics.

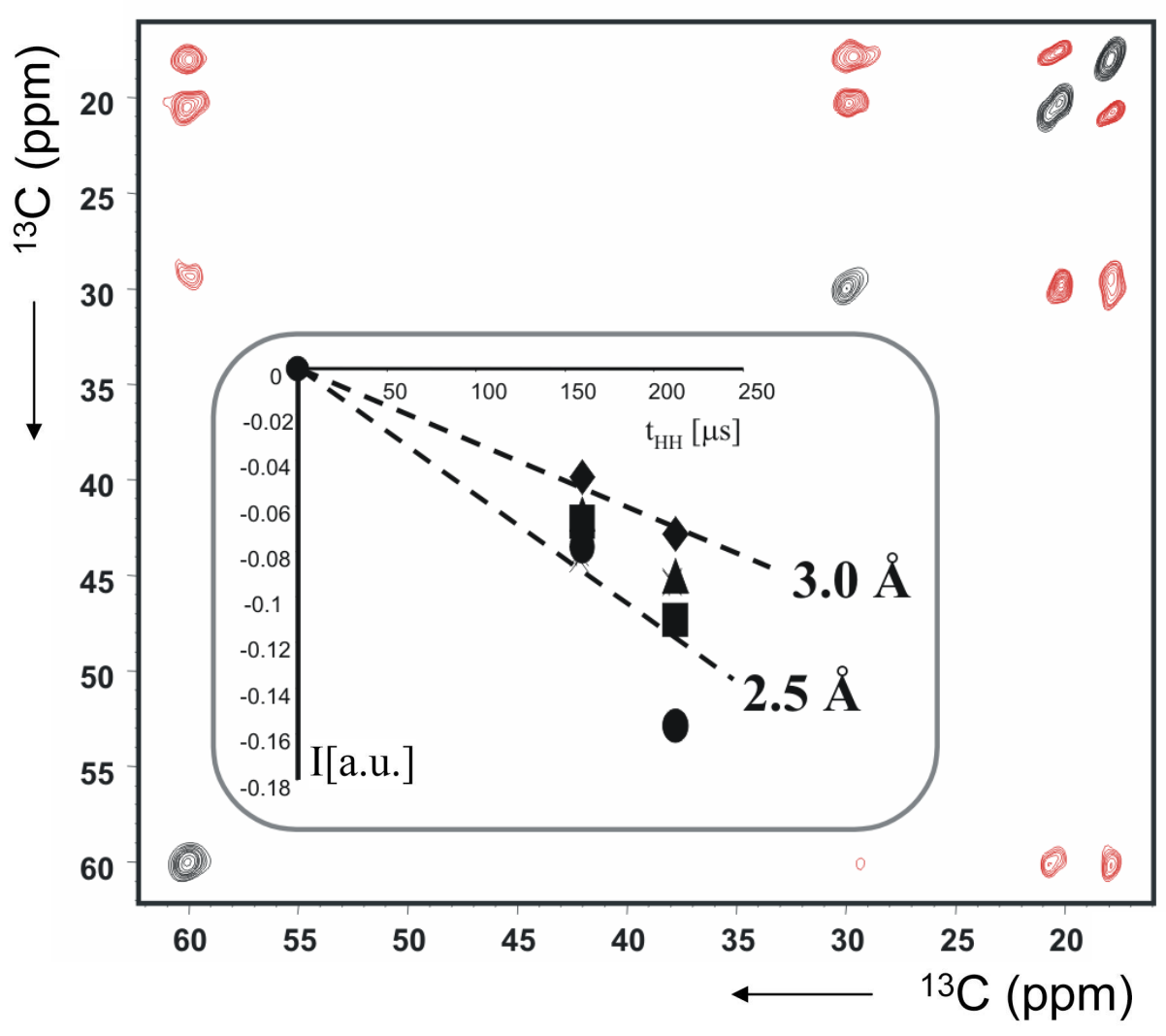

Figure 2.9. 2Q-CHHC correlation experiment on $\mathrm{U}-\left[{ }^{13} \mathrm{C},{ }^{15} \mathrm{~N}\right]$ labeled L-Valine for $t_{\mathrm{CH}}=250 \mu$ s and a proton-proton mixing time of $208 \mu \mathrm{s}$. Negative signal intensities are given in red. Insert: Symbols (see Figure 2.7) relate to experimentally detected data points. Dashed lines correspond to initial rate transfer as obtained within a quantum mechanical 2 -spin simulation for the designated ${ }^{1} \mathrm{H}-{ }^{1} \mathrm{H}$ distances.

Figure 2.9 contains experimental results obtained on $\mathrm{U}-\left[{ }^{13} \mathrm{C},{ }^{15} \mathrm{~N}\right]$ labeled L-Valine at $11 \mathrm{kHz}$ MAS for a homonuclear contact time of $208 \mu \mathrm{s}$. As expected ${ }^{90}$, all cross-peak intensities are negative relative to the positive signal values of the diagonal. In 
agreement with the crystallographic predictions (see previous section), the cross-peak amplitudes involving the $\mathrm{C} \alpha-\mathrm{C} \beta$ spin pair are considerably smaller than all other cross-peak intensities observed in the spectrum. It is possible to compare the crosspeak buildup rates in the initial rate regime to quantum mechanical two-spin simulations for inter-nuclear ${ }^{1} \mathrm{H}-{ }^{1} \mathrm{H}$ distances of $2.5 \AA$ and $3.0 \AA$. The corresponding initial linear transfer rates are indicated in Figure 2.9 as dashed lines. The NMR data are hence in good agreement with the X-ray structure. For short mixing times, (i.e., $t_{H H} \leq 0.2 \mathrm{~ms}$ ), the size of the $2 \mathrm{Q}$ cross-peak intensity reflects the inter-nuclear ${ }^{1} \mathrm{H}-{ }^{1} \mathrm{H}$ distance. For longer mixing times, strongly coupled systems require the inclusion of further proton spins within the simulation and the observed transfer amplitude changes sign in close analogy to the ${ }^{13} \mathrm{C}-{ }^{13} \mathrm{C}$ case ${ }^{91}$.

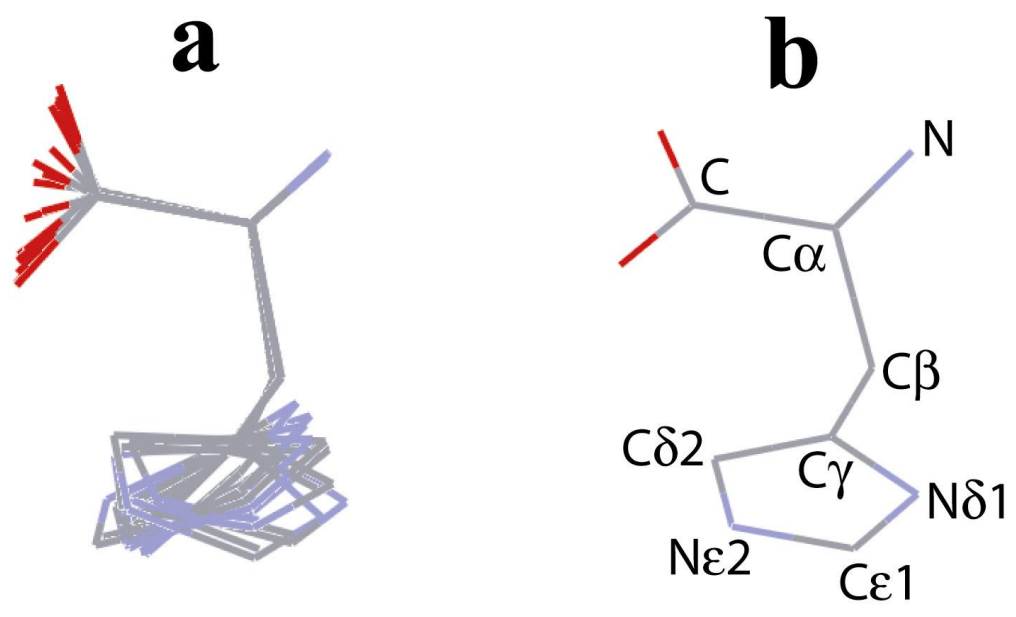

Figure 2.10. Comparison of (a) a representative ensemble of 10 structures of Histidine $\cdot \mathrm{HCl}$ using $0 \mathrm{Q}-$ CHHC constraints with (b) the published conformation obtained from neutron diffraction ${ }^{77}$. The structures were aligned along all heavy atoms excluding oxygen and aromatic ring atoms using MOLMOL $^{92}$. Note that the spread of the solid-state NMR structures in the ring is caused by a lack of restraints and not by dynamics.

\subsection{Molecular 3D structures from CHHC / NHHC constraints}

In the following it will be demonstrated in two simple cases how the structural constraints obtained from CHHC / NHHC correlation spectra can be used in the context of a standard three-dimensional structure determination. In both cases, uniformly labeled samples were considered within an unlabeled background, hence 
excluding contributions from inter-molecular ${ }^{1} \mathrm{H}-{ }^{1} \mathrm{H}$ transfer. Such interactions can provide valuable structural information but would complicate the interpretation of the transfer dynamics in terms of the $3 \mathrm{D}$ molecular structure. For sensitivity reasons ${ }^{53}$, the following results were obtained using the 0Q-CHHC / NHHC LM method.

L-Histidine $\cdot \mathrm{HCl}$. As a cross-validation, the CHHC data shown in Figure 2.6 were used to establish a set of structural constraints for L-Histidine $\cdot \mathrm{HCl}$. On the basis of the analysis presented in section 2.5 , distance constraints $\left[r_{\mathrm{ij}}{ }^{l}, r_{\mathrm{ij}}{ }^{u}\right]$ of $[1.8 \AA, 2.3 \AA]$ for the $\mathrm{C} \alpha-\mathrm{C} \beta$ pair and $[2.6 \AA, 10 \AA]$ for all other considered ${ }^{1} \mathrm{H}-{ }^{1} \mathrm{H}$ contacts were assumed. Thus, the 10 lowest-energy structures shown in Figure 2.10a were obtained using in total 6 non-trivial ${ }^{1} \mathrm{H}^{-1} \mathrm{H}$ distance constraints. The agreement to the conformation obtained from neutron diffraction ${ }^{77}$ (Figure $2.10 \mathrm{~b}$ ) could be further improved by a detailed analysis of 2Q-CHHC experiments that allow for a clear distinction between direct and relay mechanisms.

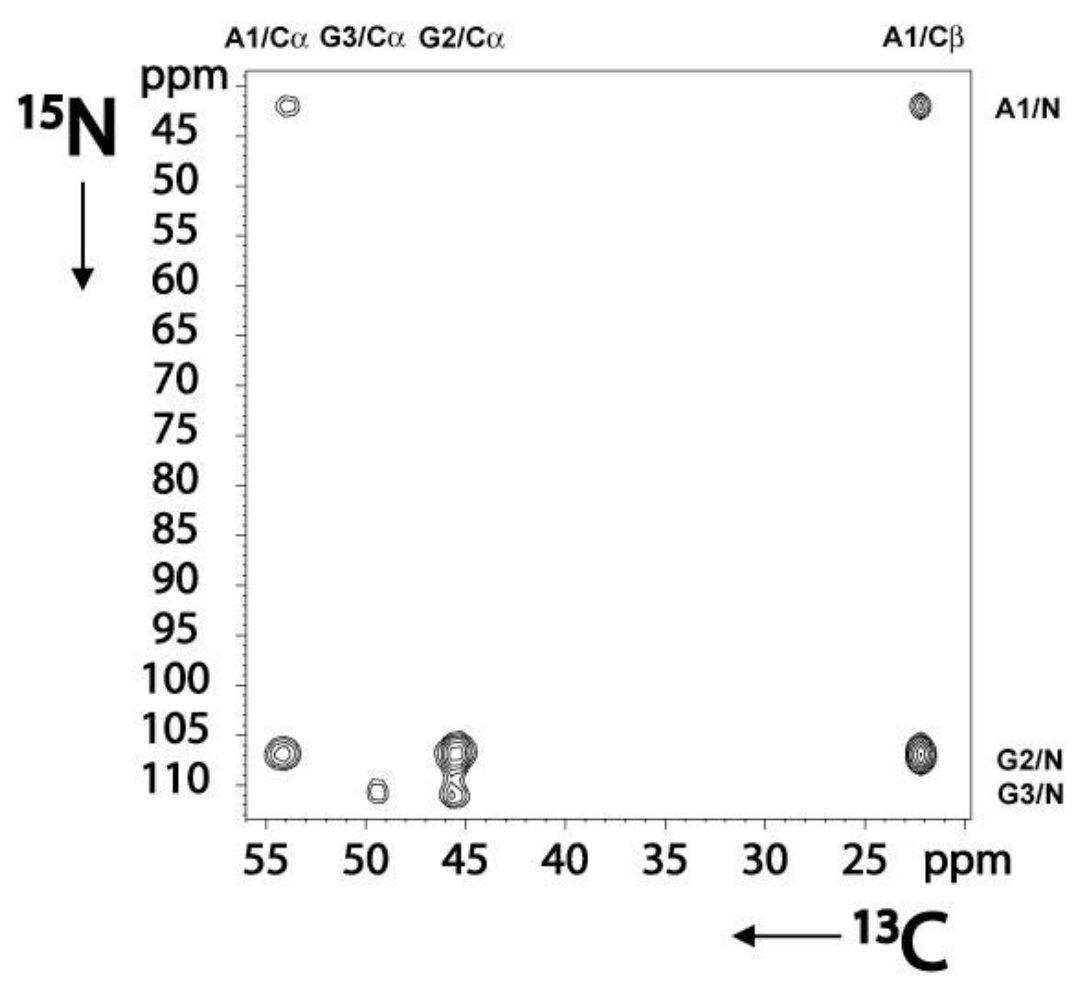

Figure 2.11. $0 \mathrm{Q}-\mathrm{NHHC}$ correlation experiment on $3 \mathrm{mg}$ of $\mathrm{U}-\left[{ }^{13} \mathrm{C},{ }^{15} \mathrm{~N}\right]$ labeled AGG for a $\mathrm{LM}$ correlation time of $200 \mu \mathrm{s}$. The experiment was conducted at $9.4 \mathrm{~T}$ and $11 \mathrm{kHz}$ MAS. $t_{\mathrm{NH}}$ was set to $500 \mu$ s. Resonance assignments were obtained from $\operatorname{ref}^{62}$. All peaks reflect ${ }^{1} \mathrm{H}-{ }^{1} \mathrm{H}$ interactions, encoded in ${ }^{15} \mathrm{~N}$ evolution and ${ }^{13} \mathrm{C}$ detection periods. 
Ala-Gly-Gly. To assess the usefulness of the presented approach in peptides, NMR results obtained on the uniformly $\left[{ }^{13} \mathrm{C},{ }^{15} \mathrm{~N}\right]$ labeled tripeptide Ala-Gly-Gly, for which a crystallographic reference structure is available ${ }^{93}$, were considered next. In addition to the CHHC-type of experiments, also a series of NHHC correlation experiments was recorded. As an example, Figure 2.11 contains results of a LM 2D experiment with $t_{\mathrm{HH}}=200 \mu \mathrm{s}$. Again, only protonated rare-spins are considered, and it is hence sufficient to concentrate on the aliphatic region of the spectrum. Similar to the homonuclear $\mathrm{CHHC}$ case, one immediately observes significant differences regarding the intensity of the observed correlations. These qualitative observations are supported by an analysis of the resulting CHHC (a) and NHHC (b) buildup curves on AGG shown in Figure 2.12.

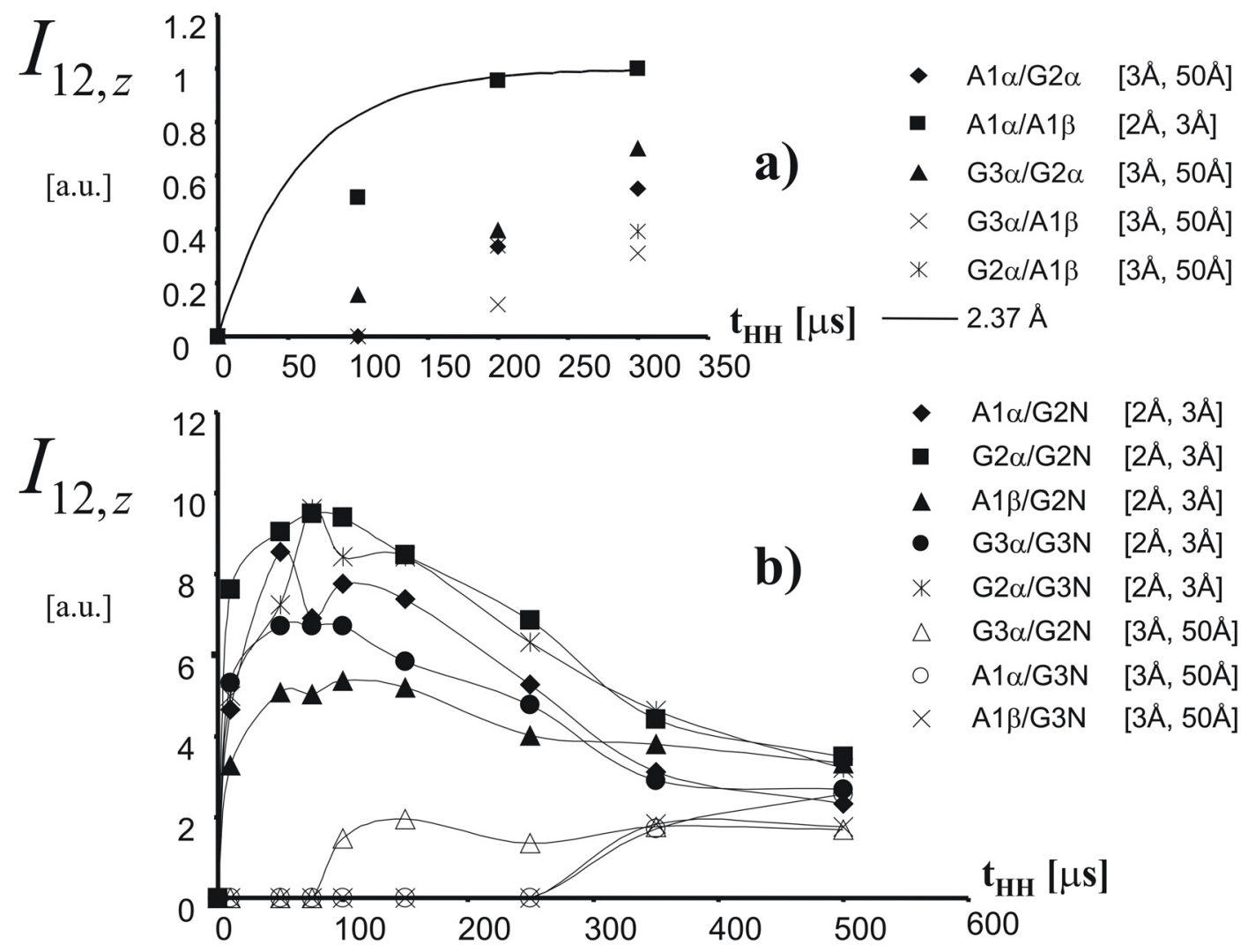

Figure 2.12. (a) CHHC buildup curves for the indicated cross-peaks. The solid line corresponds to a theoretical prediction (see Figure 2.8) using a ${ }^{1} \mathrm{H}-{ }^{1} \mathrm{H}$ distance of $2.37 \AA$, (b) $0 \mathrm{Q}-\mathrm{NHHC}$ signal buildup for the indicated ${ }^{15} \mathrm{~N}-{ }^{13} \mathrm{C}$ pairs. For the sake of clarification, connecting lines are shown. All experiments were conducted using the LM scheme (Figure 2.3a) on $3 \mathrm{mg}$ of $\mathrm{U}-\left[{ }^{13} \mathrm{C},{ }^{15} \mathrm{~N}\right]$ labeled AGG at $11 \mathrm{kHz}$ MAS and $400 \mathrm{MHz}\left({ }^{1} \mathrm{H}\right.$ resonance frequency). Resonance assignments were obtained from ref $^{62}$. Distance constraints used during the structure calculations are indicated. 
From the CHHC data presented in (a), one can easily identify one short ${ }^{1} \mathrm{H}-{ }^{1} \mathrm{H}$ distance for the A1C $\alpha$ - A1C $\beta$ pair. Notably, the initial rate buildup agrees well with a theoretical approximation of Figure 2.8 assuming the ${ }^{1} \mathrm{H}-{ }^{1} \mathrm{H}$ distance of the corresponding X-ray structure. In contrast to the CHHC correlations, in Figure $2.12 \mathrm{~b}$ local maxima for all, according to the X-ray structure, short (i.e., $2.6 \AA \pm 0.25 \AA$ ) NHHC correlations are detected. For longer mixing times, all signal intensities adopt similar values. The qualitative difference between NHHC and CHHC spectra most likely results from a different degree of total ${ }^{1} \mathrm{H}$ polarization in the sample. In (b) only $\mathrm{NH}$ protons are initially polarized and magnetization must be redistributed within the protonated ${ }^{13} \mathrm{C}$ network. As a result, transfer to nearest neighbor ${ }^{13} \mathrm{C}$ is influenced by other protons in close spatial proximity. These experimental results are in qualitative agreement with quantum-mechanical calculations of an $\mathrm{IS}_{2}$ spin system ${ }^{64}$. Comparison of Figure 2.11 and Figure 2.12 indicates that one 2D correlation experiment is sufficient to identify nearest neighbor NHHC correlations. Two of the detected interresidue correlations involve $\mathrm{C} \alpha(\mathrm{i})-\mathrm{NH}(\mathrm{i}+1)$ pairs strongly indicative ${ }^{37}$ for a $\beta$-strand arrangement of AGG predicted from the crystal structure. In a simplified, dual constraint classification, strong peaks in Figure 2.11 are attributed to NHHC distances of [2.0 $\AA, 3.0 \AA]$ and all other weak or missing correlations to ${ }^{1} \mathrm{H}-{ }^{1} \mathrm{H}$ distances of $[3.0$ $\AA$, $50.0 \AA$ ]. Similar to the study involving L-Histidine $\cdot \mathrm{HCl}$, selecting a large upper structural bound appropriately reflects the accuracy of the NMR-detected distance constraints and minimizes complications due to relay effects during the structure calculation. Note that in contrast to approaches involving chemical shift selective transfer methods, 12 non-trivial distance constraints were hence obtained from a single $2 \mathrm{D}$ experiment.

These distance constraints can provide the basis for the computation of an ensemble of $3 \mathrm{D}$ structures. In addition to these distance constraints, secondary chemical shifts strongly correlate with polypeptide backbone structure ${ }^{62,94-96}$ and provide a convenient means for establishing dihedral angle constraints in a polypeptide ${ }^{8,9,76,97}$. In particular, it was recently shown that solution-state random coil chemical shifts can be used as a standard reference to study secondary chemical shifts of uniformly labeled proteins in the solid-state ${ }^{62}$. As a result, programs such as $\operatorname{TALOS}^{76}$, originally developed for the structural analysis of conformation-dependent chemical shifts in the solution-state, 
can be used for solid-state NMR applications ${ }^{4,25,26,97-99}$. In the case of $A_{1} G_{2} G_{3}$, one finds for the central residue $\left(\mathrm{G}_{2}\right)$ backbone dihedral angles $\phi=(-104 \pm 23)^{\circ}$ and $\psi=(158$ $\pm 24)^{\circ}$, which is in agreement with the X-ray structure $\left(\phi=-83^{\circ} ; \psi=170^{\circ}\right)$ and provides an independent additional structural constraint. For the particular case of AGG, a structure calculation with CNS using only these dihedral angle constraints leads to the structure shown in Figure 2.13a and a backbone RMSD to mean of $0.51 \AA$. As expected, $\mathrm{C}$ - and $\mathrm{N}$ - terminal segments of the peptide remain poorly defined and additional constraints are necessary. In Figure 2.13b, NHHC distance parameters were supplemented as additional constraints leading to an overall reduction of the backbone RMSD to $0.37 \AA$ (RMSD to X-ray: $0.38 \AA$ ). Constraints derived from the NHHC correlation experiment are particularly useful to restrict the $\mathrm{N}$ terminus and the side chain. On the other hand, the C-terminus remains, in the absence of detectable ${ }^{1} \mathrm{H}-{ }^{1} \mathrm{H}$ contacts, poorly defined. Correspondingly, the beneficial effect of the ${ }^{1} \mathrm{H}-{ }^{1} \mathrm{H}$ constraints becomes apparent when RMSD values are calculated without the $\mathrm{C}$ terminal $\mathrm{C}^{\prime}$ atom. In this case, the RMSD value decreases from $0.39 \AA$ (TALOS) to $0.17 \AA$ (TALOS + NHHC) (RMSD to X-ray: $0.17 \AA$ ). Thus, the combination of the ${ }^{13} \mathrm{C}$ resonance assignments and a single $\mathrm{NHHC}$ experiment was sufficient to significantly restrain the $3 \mathrm{D}$ structure of AGG in the solid-state. Because only one side chain resonance can be detected, CHHC distance constraints do, in the current context, not lead to significant increase in structural accuracy.

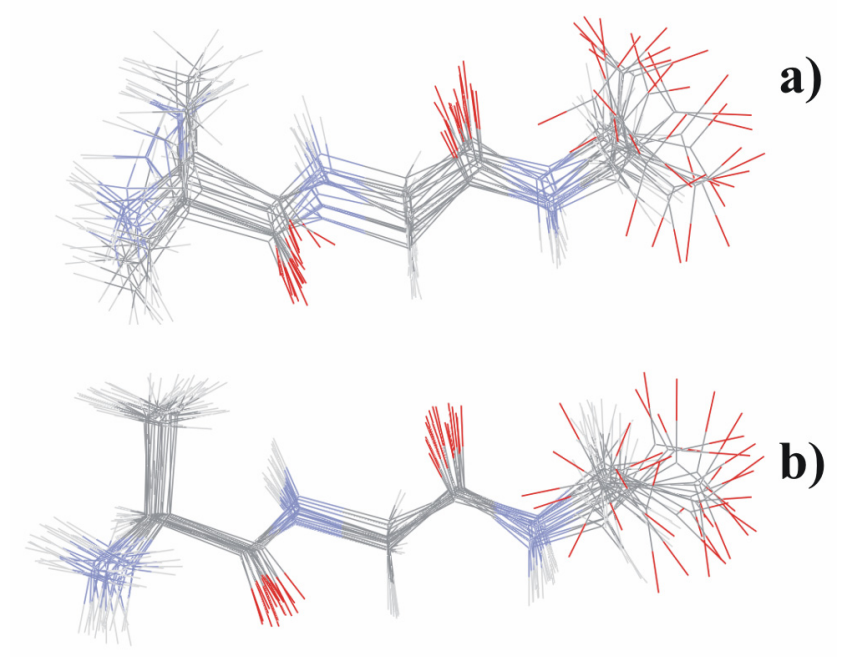

Figure 2.13. Representative ensemble of 20 structures of AGG using (a) TALOS dihedral angle constraints only and (b) their combination with NHHC distance constraints. The structures were aligned along the backbone atoms of all three residues using $\mathrm{MOLMOL}^{92}$. 


\subsection{Conclusions}

The detection of through-space contacts represents one of the primary instruments of NMR to probe structural parameters in solution or solid-state. In many applications of chemical or biological relevance, the system of interest is characterized by a large number of proton-proton contacts that, in contrast to rare-spin interactions, are indicative of its three-dimensional arrangement. In the solution-state, these contacts are easily detected by direct ${ }^{1} \mathrm{H}-{ }^{1} \mathrm{H}$ correlation experiments and have been of central importance for 3D structure determination. Both aspects provide a strong incentive to investigate whether a similar approach is viable for the structural analysis of solid biomolecules studied under high-resolution solid-state NMR conditions.

Although it has recently been demonstrated ${ }^{53,100}$ that CHHC / NHHC experiments can provide structural constraints in polypeptides and proteins, a general approach on how to analyze proton-proton contacts in terms of inter-nuclear distances has thus far not been presented. As confirmed in the first part of this chapter, this aspect may partially be attributed to the fact that an appropriate theoretical description of ${ }^{1} \mathrm{H}-{ }^{1} \mathrm{H}$ transfer rates generally depends on the details of the NMR experiment. The study in model compounds suggests a general framework in which ${ }^{1} \mathrm{H}-{ }^{1} \mathrm{H}$ transfer rates can be related to inter-nuclear distances for a given MAS rate and proton-proton mixing scheme.

For the two considered zero-quantum polarization transfer schemes, the spin system dynamics are well described within a semiclassical relaxation theory and lead to an exponential cross-peak buildup. For a given MAS rate and pulse scheme considered here, a uniform 0Q-line shape function can be obtained and the 0Q-transfer rates depend on the inverse $6^{\text {th }}$ power of the proton-proton distance. For 0Q-NHHC mixing, multiple-spin effects affect the transfer profile for longer mixing times. Nevertheless, the initial transfer rate remains diagnostic for the NHHC distance of interest. The same observations were made for $2 \mathrm{Q}$ rotating frame mixing, where the initial crosspeak buildup is again sensitive to the inter-nuclear distance and can be described within a quantum-mechanical two-spin analysis. In several aspects, the approach resembles a structural analysis of NOE parameters in the solution-state ${ }^{101}$ : The accuracy with which proton-proton contacts can be related to inter-nuclear distances is highest if results of several experiments are analyzed in the initial rate regime. Relay 
transfers and intensity variations can be accounted for by choosing appropriately wide bounds or by a direct refinement during the structure calculation ${ }^{74}$. Finally, 2Q-CHHC correlation spectra can, similar to ROESY experiments, help to distinguish direct from relay transfer. Hence, the presented concept should be of comparable value to NOErelated spectroscopy in the solution-state.

In two simple examples, it was demonstrated how ${ }^{1} \mathrm{H}-{ }^{1} \mathrm{H}$ constraints can be used to determine molecular structures using solid-state NMR. In contrast to previous reports, a small set of $2 \mathrm{D}$ experiments was sufficient to establish side chain or backbone conformation in two model systems. For AGG, a single NHHC correlation spectrum suffices to calculate the 3D structure to a backbone RMSD of $0.37 \AA$. This investigation provides the empirical basis for future structural studies in other protonated molecules including ligands of small molecular weight ${ }^{4}$, fibrous or membrane proteins. Here, ${ }^{1} \mathrm{H}-{ }^{1} \mathrm{H}$ contacts not only report on local backbone or side chain orientation, but they are particularly useful to establish medium and long-range constraints for the three-dimensional protein fold. It was previously shown for the 76residue protein ubiquitin that such constraints can in principle be detected using the discussed CHHC / NHHC concept ${ }^{53}$. Provided that a sufficient number of protonproton distances and dihedral angle constraints can be obtained, solid-state NMR experiments as discussed here could therefore lead to the de novo protein structure determination using a single protein sample. 
1. Long, J. R., Oyler, N., Drobny, G. P. \& Stayton, P. S. Assembly of $\alpha$-helical peptide coatings on hydrophobic surfaces. Journal of the American Chemical Society 124, 6297-6303 (2002).

2. Smith, S. O. et al. Structure of the transmembrane dimer interface of glycophorin A in membrane bilayers. Biochemistry 40, 6553-6558 (2001).

3. Isaac, B., Gallagher, G. J., Balazs, Y. S. \& Thompson, L. K. Site-directed rotational resonance solid-state NMR distance measurements probe structure and mechanism in the transmembrane domain of the serine bacterial chemoreceptor. Biochemistry 41, 3025-3036 (2002).

4. Luca, S. et al. The conformation of neurotensin bound to its $\mathrm{G}$ protein-coupled receptor. Proceedings of the National Academy of Sciences of the United States of America 100, 10706-10711 (2003).

5. Andronesi, O. C. et al. Determination of membrane protein structure and dynamics by magic-angle-spinning solid-state NMR spectroscopy. Journal of the American Chemical Society 127, 12965-12974 (2005).

6. van Gammeren, A. J., Hulsbergen, F. B., Hollander, J. G. \& de Groot, H. J. M. Residual backbone and side chain ${ }^{13} \mathrm{C}$ and ${ }^{15} \mathrm{~N}$ resonance assignments of the intrinsic transmembrane light-harvesting 2 protein complex by solid-state magic-angle-spinning NMR spectroscopy. Journal of Biomolecular NMR 31, 279-293 (2005).

7. van Beek, J. D., Hess, S., Vollrath, F. \& Meier, B. H. The molecular structure of spider dragline silk: Folding and orientation of the protein backbone. Proceedings of the National Academy of Sciences of the United States of America 99, 10266-10271 (2002).

8. Petkova, A. T. et al. A structural model for Alzheimer's $\beta$-amyloid fibrils based on experimental constraints from solid-state NMR. Proceedings of the National Academy of Sciences of the United States of America 99, 1674216747 (2002).

9. Jaroniec, C. P., MacPhee, C. E., Astrof, N. S., Dobson, C. M. \& Griffin, R. G. Molecular conformation of a peptide fragment of transthyretin in an amyloid fibril. Proceedings of the National Academy of Sciences of the United States of America 99, 16748-16753 (2002).

10. Petkova, A. T. et al. Self-propagating, molecular-level polymorphism in Alzheimer's $\beta$-amyloid fibrils. Science 307, 262-265 (2005).

11. Ritter, C. et al. Correlation of structural elements and infectivity of the HET-s prion. Nature 435, 844-848 (2005).

12. Siemer, A. B., Ritter, C., Ernst, M., Riek, R. \& Meier, B. H. High-resolution solid-state NMR spectroscopy of the prion protein HET-s in its amyloid conformation. Angewandte Chemie-International Edition 44, 2441-2444 (2005).

13. Heise, H. et al. Molecular-level secondary structure, polymorphism, and dynamics of full-length $\alpha$-synuclein fibrils studied by solid-state NMR. Proceedings of the National Academy of Sciences of the United States of America 102, 15871-15876 (2005).

14. Jaroniec, C. P. et al. High-resolution molecular structure of a peptide in an amyloid fibril determined by magic-angle-spinning NMR spectroscopy. Proceedings of the National Academy of Sciences of the United States of America 101, 711-716 (2004).

15. Thompson, L. K. Solid-state NMR studies of the structure and mechanisms of proteins. Current Opinion in Structural Biology 12, 661-669 (2002). 
16. McDermott, A. E. Structural and dynamic studies of proteins by solid-state NMR spectroscopy: Rapid movement forward. Current Opinion in Structural Biology 14, 554-561 (2004).

17. Tycko, R. Applications of solid-state NMR to the structural characterization of amyloid fibrils: Methods and results. Progress in Nuclear Magnetic Resonance Spectroscopy 42, 53-68 (2003).

18. Luca, S., Heise, H. \& Baldus, M. High-resolution solid-state NMR applied to polypeptides and membrane proteins. Accounts of Chemical Research 36, 858865 (2003).

19. Luca, S., Heise, H., Lange, A. \& Baldus, M. Investigation of ligand-receptor systems by high-resolution solid-state NMR: Recent progress and perspectives. Archiv der Pharmazie 338, 217-228 (2005).

20. Smith, S. O., Aschheim, K. \& Groesbeek, M. Magic-angle-spinning NMR spectroscopy of membrane proteins. Quarterly Reviews of Biophysics 29, 395449 (1996).

21. LeMaster, D. M. \& Kushlan, D. M. Dynamical mapping of E. coli thioredoxin via ${ }^{13} \mathrm{C}$ NMR relaxation analysis. Journal of the American Chemical Society 118, 9255-9264 (1996).

22. Gardner, K. H. \& Kay, L. E. The use of ${ }^{2} \mathrm{H},{ }^{13} \mathrm{C},{ }^{15} \mathrm{~N}$ multi-dimensional NMR to study the structure and dynamics of proteins. Annual Review of Biophysics and Biomolecular Structure 27, 357-406 (1998).

23. Hong, M. \& Jakes, K. Selective and extensive ${ }^{13} \mathrm{C}$ labeling of a membrane protein for solid-state NMR investigations. Journal of Biomolecular NMR 14, 71-74 (1999).

24. Castellani, F. et al. Structure of a protein determined by solid-state magicangle-spinning NMR spectroscopy. Nature 420, 98-102 (2002).

25. Nomura, K., Takegoshi, K., Terao, T., Uchida, K. \& Kainosho, M. Determination of the complete structure of a uniformly labeled molecule by rotational resonance solid-state NMR in the tilted rotating frame. Journal of the American Chemical Society 121, 4064-4065 (1999).

26. Rienstra, C. M. et al. De novo determination of peptide structure with solidstate magic-angle-spinning NMR spectroscopy. Proceedings of the National Academy of Sciences of the United States of America 99, 10260-10265 (2002).

27. Baldus, M., Petkova, A. T., Herzfeld, J. \& Griffin, R. G. Cross-polarization in the tilted frame: Assignment and spectral simplification in heteronuclear spin systems. Molecular Physics 95, 1197-1207 (1998).

28. Jaroniec, C. P. et al. Measurement of dipolar couplings in a uniformly $\left[{ }^{13} \mathrm{C}\right.$, $\left.{ }^{15} \mathrm{~N}\right]$ labeled membrane protein: Distances between the Schiff base and aspartic acids in the active site of bacteriorhodopsin. Journal of the American Chemical Society 123, 12929-12930 (2001).

29. Sonnenberg, L., Luca, S. \& Baldus, M. Multiple-spin analysis of chemical shift selective ${ }^{13} \mathrm{C}-{ }^{13} \mathrm{C}$ transfer in uniformly labeled biomolecules. Journal of Magnetic Resonance 166, 100-110 (2004).

30. Baldus, M. \& Meier, B. H. Broadband polarization transfer under magicangle-spinning: Application to total through-space correlation NMR spectroscopy. Journal of Magnetic Resonance 128, 172-193 (1997).

31. Kiihne, S. et al. Distance measurements by dipolar recoupling twodimensional solid-state NMR. Journal of Physical Chemistry A 102, 22742282 (1998). 
32. Bennett, A. E. et al. Homonuclear radio frequency-driven recoupling in rotating solids. Journal of Chemical Physics 108, 9463-9479 (1998).

33. Kiihne, S. R. et al. Distance measurements in multiply labeled crystalline cytidines by dipolar recoupling solid-state NMR. Journal of Physical Chemistry A 103, 3890-3903 (1999).

34. Goobes, G., Boender, G. J. \& Vega, S. Spinning-frequency dependent narrowband RF-driven dipolar recoupling. Journal of Magnetic Resonance 146, 204-219 (2000).

35. Helluy, X., Marichal, C. \& Sebald, A. Through-bond indirect and throughspace direct dipolar coupling ${ }^{31} \mathrm{P}$ MAS NMR constraints for spectral assignment in the cubic $3 \times 3 \times 3$ superstructure of $\mathrm{TiP}_{2} \mathrm{O}_{7}$. Journal of Physical Chemistry B 104, 2836-2845 (2000).

36. Goobes, G. \& Vega, S. Improved narrowband dipolar recoupling for homonuclear distance measurements in rotating solids. Journal of Magnetic Resonance 154, 236-251 (2002).

37. Wüthrich, K. NMR of proteins and nucleic acids (Wiley Interscience, New York, 1986).

38. Ernst, R. R., Bodenhausen, G. \& Wokaun, A. Principles of nuclear magnetic resonance in one and two dimensions (Clarendon Press, Oxford, 1987).

39. Cavanagh, J., Fairbrother, W. J., Palmer, A. G. \& Skelton, N. J. Protein NMR spectroscopy, principles and practice (Academic Press, San Diego, 1996).

40. Solomon, I. Relaxation processes in a system of two spins. Physical Review 99, 559-565 (1955).

41. Macura, S. \& Ernst, R. R. Elucidation of cross-relaxation in liquids by twodimensional NMR spectroscopy. Molecular Physics 41, 95-117 (1980).

42. Kumar, A., Wagner, G., Ernst, R. R. \& Wüthrich, K. Buildup rates of the nuclear Overhauser effect measured by two-dimensional proton magnetic resonance spectroscopy - Implications for studies of protein conformation. Journal of the American Chemical Society 103, 3654-3658 (1981).

43. Jeener, J., Meier, B. H., Bachmann, P. \& Ernst, R. R. Investigation of exchange processes by two-dimensional NMR spectroscopy. Journal of Chemical Physics 71, 4546-4553 (1979).

44. Bothner-By, A. A., Stephens, R. L., Lee, J. M., Warren, C. D. \& Jeanloz, R. W. Structure determination of a tetrasaccharide - Transient nuclear Overhauser effects in the rotating frame. Journal of the American Chemical Society 106, 811-813 (1984).

45. Bax, A. \& Davis, D. G. Practical aspects of two-dimensional transverse NOE spectroscopy. Journal of Magnetic Resonance 63, 207-213 (1985).

46. Griesinger, C. \& Ernst, R. R. Frequency offset effects and their elimination in NMR rotating frame cross-relaxation spectroscopy. Journal of Magnetic Resonance 75, 261-271 (1987).

47. Caravatti, P., Braunschweiler, L. \& Ernst, R. R. Heteronuclear correlation spectroscopy in rotating solids. Chemical Physics Letters 100, 305-310 (1983).

48. Schnell, I. \& Spiess, H. W. High-resolution ${ }^{1}$ H NMR spectroscopy in the solid-state: Very fast sample rotation and multiple-quantum coherences. Journal of Magnetic Resonance 151, 153-227 (2001).

49. Zhang, S., Meier, B. H. \& Ernst, R. R. Polarization echoes in NMR. Physical Review Letters 69, 2149-2151 (1992). 
50. Tomaselli, M., Hediger, S., Suter, D. \& Ernst, R. R. Nuclear magnetic resonance polarization and coherence echoes in static and rotating solids. Journal of Chemical Physics 105, 10672-10681 (1996).

51. Wilhelm, M., Feng, H., Tracht, U. \& Spiess, H. W. 2D CP/MAS ${ }^{13}$ C isotropic chemical shift correlation established by ${ }^{1} \mathrm{H}$ spin diffusion. Journal of Magnetic Resonance 134, 255-260 (1998).

52. Mulder, F. M., Heinen, W., van Duin, M., Lugtenburg, J. \& de Groot, H. J. M. Spin diffusion with ${ }^{13} \mathrm{C}$ selection and detection for the characterization of morphology in labeled polymer blends with MAS NMR. Journal of the American Chemical Society 120, 12891-12894 (1998).

53. Lange, A., Luca, S. \& Baldus, M. Structural constraints from proton-mediated rare-spin correlation spectroscopy in rotating solids. Journal of the American Chemical Society 124, 9704-9705 (2002).

54. de Boer, I., Bosman, L., Raap, J., Oschkinat, H. \& de Groot, H. J. M. 2D ${ }^{13}$ C${ }^{13} \mathrm{C}$ MAS NMR correlation spectroscopy with mixing by true ${ }^{1} \mathrm{H}$ spin diffusion reveals long-range inter-molecular distance restraints in ultra-high magnetic field. Journal of Magnetic Resonance 157, 286-291 (2002).

55. Suter, D. \& Ernst, R. R. Spectral spin diffusion in the presence of an extraneous dipolar reservoir. Physical Review B 25, 6038-6041 (1982).

56. Suter, D. \& Ernst, R. R. Spin diffusion in resolved solid-state NMR spectra. Physical Review B 32, 5608-5627 (1985).

57. Kubo, A. \& McDowell, C. A. Spectral spin diffusion in polycrystalline solids under magic-angle-spinning. Journal of the Chemical Society-Faraday Transactions I 84, 3713-3730 (1988).

58. Smith, S. A., Levante, T. O., Meier, B. H. \& Ernst, R. R. Computer simulations in magnetic resonance - An object-oriented programming approach. Journal of Magnetic Resonance Series A 106, 75-105 (1994).

59. Haeberlen, U. in: Advances in Magnetic Resonance (Academic Press, New York, 1976).

60. Tesche, B. \& Haeberlen, U. Proton chemical shift tensors of methyl groups; a multiple-pulse NMR and LORG/IGLO ab initio study. Journal of Magnetic Resonance Series A 117, 186-192 (1995).

61. Mehring, M. Principles of high-resolution NMR in solids (Springer, Berlin, 1983).

62. Luca, S. et al. Secondary chemical shifts in immobilized peptides and proteins: A qualitative basis for structure refinement under magic-angle-spinning. Journal of Biomolecular NMR 20, 325-331 (2001).

63. Baldus, M., Levante, T. O. \& Meier, B. H. Numerical simulation of magnetic resonance experiments - Concepts and applications to static, rotating and double rotating experiments. Zeitschrift für Naturforschung 49, 80-88 (1994).

64. Baldus, M. Correlation experiments for assignment and structure elucidation of immobilized polypeptides under magic-angle-spinning. Progress in Nuclear Magnetic Resonance Spectroscopy 41, 1-47 (2002).

65. Oas, T. G., Griffin, R. G. \& Levitt, M. H. Rotary resonance recoupling of dipolar interactions in solid-state nuclear magnetic resonance spectroscopy. Journal of Chemical Physics 89, 692-695 (1988).

66. Gan, Z. H. \& Grant, D. M. Rotational resonance in a spin-lock field for solidstate NMR. Chemical Physics Letters 168, 304-308 (1990). 
67. Nielsen, N. C., Creuzet, F., Griffin, R. G. \& Levitt, M. H. Enhanced doublequantum nuclear magnetic resonance in spinning solids at rotational resonance. Journal of Chemical Physics 96, 5668-5677 (1992).

68. Verel, R., Baldus, M., Ernst, M. \& Meier, B. H. A homonuclear spin-pair filter for solid-state NMR based on adiabatic-passage techniques. Chemical Physics Letters 287, 421-428 (1998).

69. Gullion, T. \& Vega, S. A simple magic-angle-spinning NMR experiment for the dephasing of rotational echoes of dipolar coupled homonuclear spin pairs. Chemical Physics Letters 194, 423-428 (1992).

70. Bennett, A. E., Ok, J. H., Griffin, R. G. \& Vega, S. Chemical shift correlation spectroscopy in rotating solids - Radio frequency-driven dipolar recoupling and longitudinal exchange. Journal of Chemical Physics 96, 8624-8627 (1992).

71. Hohwy, M., Jakobsen, H. J., Eden, M., Levitt, M. H. \& Nielsen, N. C. Broadband dipolar recoupling in the nuclear magnetic resonance of rotating solids: A compensated C7 pulse sequence. Journal of Chemical Physics 108, 2686-2694 (1998).

72. Brink, D. M. \& Satchler, G. R. Angular momentum (Oxford University press, Oxford, 1961).

73. Brunger, A. T. et al. Crystallography \& NMR system: A new software suite for macromolecular structure determination. Acta Crystallographica Section D-Biological Crystallography 54, 905-921 (1998).

74. Nilges, M. Structure calculation from NMR data. Current Opinion in Structural Biology 6, 617-623 (1996).

75. Fletcher, C. M., Jones, D. N. M., Diamond, R. \& Neuhaus, D. Treatment of NOE constraints involving equivalent or non-stereoassigned protons in calculations of biomacromolecular structures. Journal of Biomolecular NMR 8, 292-310 (1996).

76. Cornilescu, G., Delaglio, F. \& Bax, A. Protein backbone angle restraints from searching a database for chemical shift and sequence homology. Journal of Biomolecular NMR 13, 289-302 (1999).

77. Hohlwein, D. Photographic neutron-diffraction study of L-histidine $\cdot \mathrm{HCl} \cdot \mathrm{H}_{2} \mathrm{O}$ by the modified Laue method. Acta Crystallographica Section A 33, 649-654 (1977).

78. Henrichs, P. M., Linder, M. \& Hewitt, J. M. Dynamics of the ${ }^{13}$ C spinexchange process in solids - A theoretical and experimental study. Journal of Chemical Physics 85, 7077-7086 (1986).

79. Brunner, E., Freude, D., Gerstein, B. C. \& Pfeifer, H. Residual linewidths of NMR spectra of spin-1/2 systems under magic-angle-spinning. Journal of Magnetic Resonance 90, 90-99 (1990).

80. Samoson, A., Tuherm, T. \& Gan, Z. High-field high-speed MAS resolution enhancement in ${ }^{1} \mathrm{H}$ NMR spectroscopy of solids. Solid-state Nuclear Magnetic Resonance 20, 130-136 (2001).

81. Torii, K. \& Iitaka, Y. The crystal structure of L-valine. Acta Crystallographica Section B-Structural Crystallography and Crystal Chemistry 26, 1317-1326 (1970).

82. Lane, A. N. The influence of spin diffusion and internal motions on NOE intensities in proteins. Journal of Magnetic Resonance 78, 425-439 (1988).

83. Olejniczak, E. T. Including methyl rotation in simulations of spin-lattice relaxation experiments. Journal of Magnetic Resonance 81, 392-394 (1989). 
84. Liu, H., Thomas, P. D. \& James, T. L. Averaging of cross-relaxation rates and distances for methyl, methylene, and aromatic ring protons due to motion or overlap - Extraction of accurate distances iteratively via relaxation matrix analysis of 2D NOE spectra. Journal of Magnetic Resonance 98, 163-175 (1992).

85. Koning, T. M. G., Boelens, R. \& Kaptein, R. Calculation of the nuclear Overhauser effect and the determination of proton-proton distances in the presence of internal motions. Journal of Magnetic Resonance 90, 111-123 (1990).

86. Fritzhanns, T., Demco, D. E., Hafner, S. \& Spiess, H. W. Multi-dimensional ${ }^{1}$ H NMR nuclear Overhauser spectroscopy under magic-angle-spinning: Theory and application to elastomers. Molecular Physics 97, 931-943 (1999).

87. Noggle, J. H. \& Schirmer, R. E. The nuclear Overhauser effect (Academic Press, New York, 1971).

88. Massefski, W. \& Bolton, P. H. Quantitative analysis of nuclear Overhauser effects. Journal of Magnetic Resonance 65, 526-530 (1985).

89. Neuhaus, D. \& Williamson, M. The nuclear Overhauser effect in structural and conformational analysis (Wiley-VCH, New York, 1989).

90. Baldus, M., Tomaselli, M., Meier, B. H. \& Ernst, R. R. Broadband polarization-transfer experiments for rotating solids. Chemical Physics Letters 230, 329-336 (1994).

91. Sun, B. Q., Costa, P. R., Kocisko, D., Lansbury, P. T. \& Griffin, R. G. Internuclear distance measurements in solid-state nuclear magnetic resonance Dipolar recoupling via rotor synchronized spin locking. Journal of Chemical Physics 102, 702-707 (1995).

92. Koradi, R., Billeter, M. \& Wüthrich, K. MOLMOL: A program for display and analysis of macromolecular structures. Journal of Molecular Graphics 14, 51-55 (1996).

93. Subramanian, E. \& Lalitha, V. Crystal structure of a tripeptide, L-AlanylGlycyl-Glycine and its relevance to the poly(Glycine)-II type of conformation. Biopolymers 22, 833-838 (1983).

94. Saito, H. Conformation-dependent ${ }^{13} \mathrm{C}$ chemical shifts - A new means of conformational characterization as obtained by high-resolution solid-state ${ }^{13} \mathrm{C}$ NMR. Magnetic Resonance in Chemistry 24, 835-852 (1986).

95. Spera, S. \& Bax, A. Empirical correlation between protein backbone conformation and $\mathrm{C} \alpha$ and $C \beta{ }^{13} \mathrm{C}$ nuclear magnetic resonance chemical shifts. Journal of the American Chemical Society 113, 5490-5492 (1991).

96. Dedios, A. C., Pearson, J. G. \& Oldfield, E. Secondary and tertiary structural effects on protein NMR chemical shifts - An ab initio approach. Science 260, 1491-1496 (1993).

97. Balbach, J. J. et al. Amyloid fibril formation by $\mathrm{A} \beta_{16-22}$, a seven-residue fragment of the Alzheimer's $\beta$-amyloid peptide, and structural characterization by solid-state NMR. Biochemistry 39, 13748-13759 (2000).

98. Ishii, Y. ${ }^{13} \mathrm{C}-{ }^{13} \mathrm{C}$ dipolar recoupling under very fast magic-angle-spinning in solid-state nuclear magnetic resonance: Applications to distance measurements, spectral assignments, and high-throughput secondary-structure determination. Journal of Chemical Physics 114, 8473-8483 (2001).

99. Böckmann, A. et al. Solid-state NMR sequential resonance assignments and conformational analysis of the $2 \times 10.4 \mathrm{kDa}$ dimeric form of the Bacillus subtilis protein Crh. Journal of Biomolecular NMR 27, 323-339 (2003). 
100. Tycko, R. \& Ishii, Y. Constraints on supramolecular structure in amyloid fibrils from two-dimensional solid-state NMR spectroscopy with uniform isotopic labeling. Journal of the American Chemical Society 125, 6606-6607 (2003).

101. Nilges, M. Ambiguous distance data in the calculation of NMR structures. Folding \& Design 2, S53-S57 (1997). 



\section{The 3D structure of uniformly labeled kaliotoxin}

\subsection{Introduction}

Despite of its significant pharmacological relevance, no general concept for studying three-dimensional (3D) biomolecular structures and interactions in non-crystalline and insoluble systems is currently available. Examples of such structures and interactions include ligands bound with high affinity to $\mathrm{G}$ protein-coupled receptors or ion channels, protein fibrils, and biopolymers. In these cases, the application of the two principal experimental methods for 3D-structure determination, X-ray crystallography and solution-state NMR spectroscopy, can be challenging. Alternatively, NMR spectroscopic techniques specifically designed for the study of immobilized, possibly membrane-associated, systems (solid-state NMR spectroscopy) can be used to infer structural or dynamic parameters (see, for example, references ${ }^{1-6}$ ).

3D molecular structures of small peptides have been probed by using solid-state NMR spectroscopic methods based on the determination of specific ${ }^{13} \mathrm{C}-{ }^{13} \mathrm{C}$ or ${ }^{13} \mathrm{C}-{ }^{15} \mathrm{~N}$ distances $^{7,8}$. These techniques can require a high degree of spectral dispersion and the recording of a large number of NMR spectra. The extension of such methods to larger polypeptides may therefore be difficult. Instead, newer methods are based on the spectral analysis of several polypeptide variants containing isotope labels at welldefined atomic positions or amino acid residue types ${ }^{5,9-11}$. Such approaches were successful for membrane-embedded proteins oriented macroscopically on glass plates $^{1,5}$, as well as for peptides ${ }^{8}$, amyloid fibrils ${ }^{9,10}$, and a microcrystalline protein ${ }^{11}$ studied under the conditions of magic-angle-spinning (MAS) ${ }^{12}$. Since these methods require the synthesis of several isotope-labeled protein variants, they are of limited use if a rapid 3D structural characterization is desired. Their application may be further complicated by difficulties in producing adequate quantities of isotope-labeled samples of a protein or (possibly non-peptidic) ligand.

In this chapter a solid-state NMR spectroscopic approach that permits the rapid determination of 3D molecular structure with a single uniformly isotope-labeled sample is presented. For analogous NMR spectroscopic investigations in the solution state, the detection of short distances between hydrogen atoms provides the principal 
source of information about through-space distances and hence about the 3D fold of the protein ${ }^{13}$. Because of the restricted spectral resolution, two-dimensional (2D) ${ }^{1} \mathrm{H}$ ${ }^{1} \mathrm{H}$ correlation methods are of limited use for solid-state NMR spectroscopy. Instead, in the following indirect detection and structural analysis of ${ }^{1} \mathrm{H}-{ }^{1} \mathrm{H}$ interactions by using ${ }^{13} \mathrm{C}-{ }^{13} \mathrm{C}$ correlation spectroscopy as described in detail in chapter 2 is utilized. Combined with dihedral-angle constraints, which can be derived from conformationdependent chemical shifts, the characterization of the 3D molecular structure from a single protein sample becomes possible. The approach is demonstrated on kaliotoxin (KTX), a 38-residue peptide found in the venom of the scorpion Androctonus mauretanicus mauretanicus $^{14}$. KTX has been shown to block voltage-dependent eukaryotic potassium channels (e.g., Shaker and Kv1.3) with high affinity and a 1:1 stoichiometry ${ }^{15}$.

\subsection{Solid-state NMR spectroscopy}

Uniformly $\left[{ }^{13} \mathrm{C},{ }^{15} \mathrm{~N}\right]$ labeled KTX was expressed recombinantly as an intein fusion protein $^{16}$ in Escherichia coli. Electrophysiological experiments measuring the blockage of KcsA-Kv1.3 potassium channels ${ }^{17}$ show that the purified KTX is fully functional. A lyophilized sample of uniformly $\left[{ }^{13} \mathrm{C},{ }^{15} \mathrm{~N}\right]$ labeled KTX was rehydrated and investigated under MAS conditions. No additional attempts were made to improve the structural homogeneity of the sample.

Two-dimensional NMR experiments were conducted on a $14.1 \mathrm{~T}\left({ }^{1} \mathrm{H}\right.$ resonance frequency: $600 \mathrm{MHz}$ ) wide-bore and an $18.8 \mathrm{~T}$ ( ${ }^{1} \mathrm{H}$ resonance frequency: $800 \mathrm{MHz}$ ) standard-bore instrument (Bruker Biospin, Germany) equipped with $4 \mathrm{~mm}$ tripleresonance $\left({ }^{1} \mathrm{H},{ }^{13} \mathrm{C},{ }^{15} \mathrm{~N}\right)$ MAS probes. All experiments were carried out at probe temperatures between $-6^{\circ} \mathrm{C}$ and $-15^{\circ} \mathrm{C}$. The MAS frequency was set to $11 \mathrm{kHz}(600$ $\mathrm{MHz})$ and $12.5 \mathrm{kHz}(800 \mathrm{MHz})$, respectively. High power proton-decoupling using the sequence SPINAL64 ${ }^{18}$ with r.f. amplitudes of $80-90 \mathrm{kHz}$ was applied during evolution and detection periods. For the sequential assignment of ${ }^{13} \mathrm{C} /{ }^{15} \mathrm{~N}$ backbone and side chain resonances, the following homo- and heteronuclear experiments were performed: 
${ }^{13} \mathrm{C}-{ }^{13} \mathrm{C}$ : A conventional proton-driven spin diffusion (SD) scheme employing longitudinal mixing times of 10, 50, 100, 150, 200 and $300 \mathrm{~ms}$ was used on the 800 $\mathrm{MHz}$ instrument (4-21 h total acquisition time). At $12.5 \mathrm{kHz}$ MAS, sequential CA-CA and CA-CB correlations can be detected due to weak C'-CA recoupling (see Figure $3.1)^{19}$. To distinguish one-bond correlations from relayed and sequential correlations, an $R 18_{8}^{5}$ double-quantum spectrum ${ }^{20}$ was recorded ( $15 \mathrm{~h}$ total acquisition time). The contact time $t_{\mathrm{HC}}$ of the ramped cross-polarization was set to $200 \mu \mathrm{s}$ in all ${ }^{13} \mathrm{C}-{ }^{13} \mathrm{C}$ experiments except for the $10 \mathrm{~ms}$ SD experiment where a CP time of $1 \mathrm{~ms}$ was used.

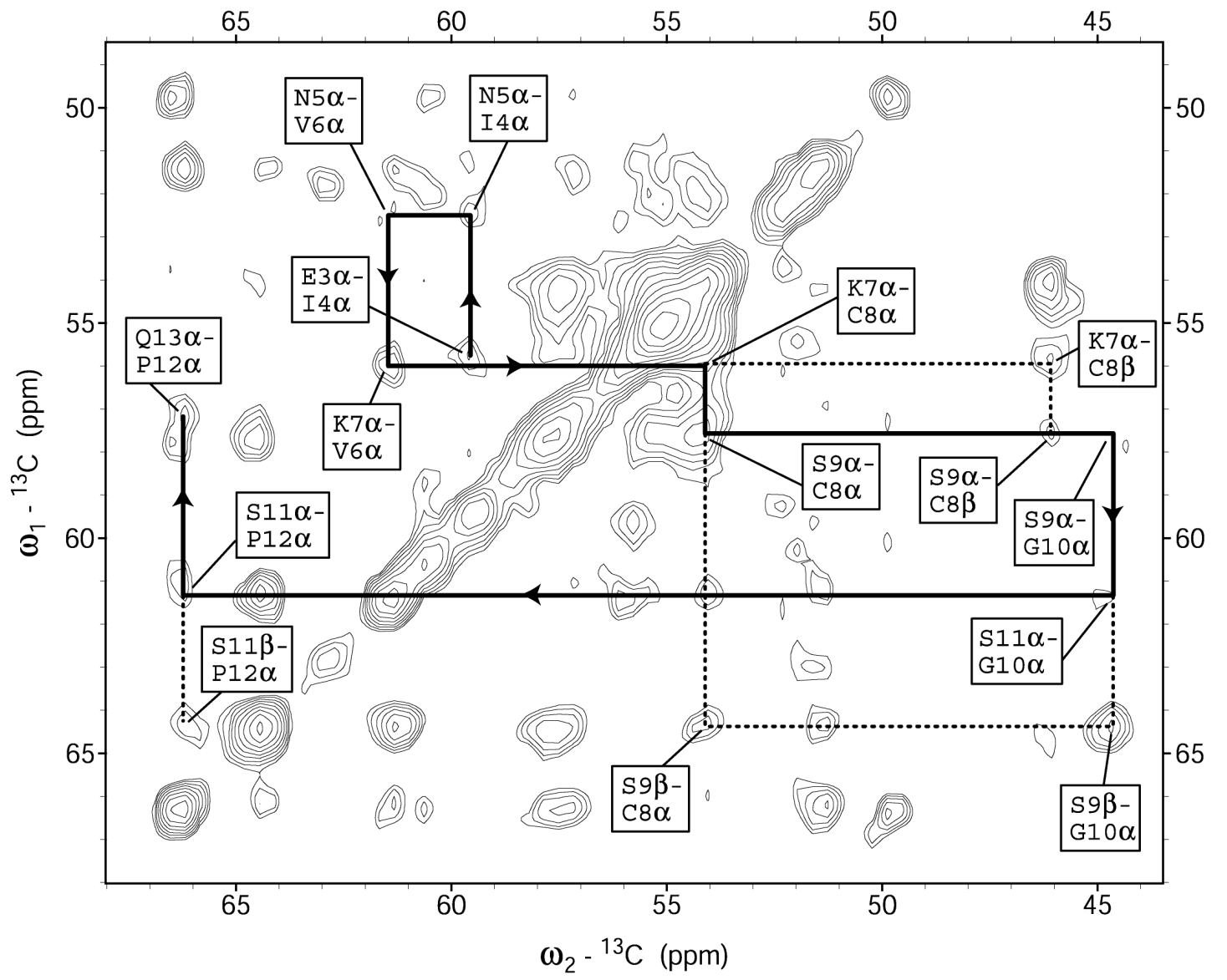

Figure 3.1. $2 \mathrm{D}{ }^{13} \mathrm{C}-{ }^{13} \mathrm{C}$ spin-diffusion spectrum (mixing time: $150 \mathrm{~ms}$ ) of $\mathrm{U}-\left[{ }^{13} \mathrm{C},{ }^{15} \mathrm{~N}\right]$ labeled KTX recorded on an $800 \mathrm{MHz}$ NMR spectrometer (Bruker Biospin, Germany) at $12.5 \mathrm{kHz}$ MAS. The assigned sequential CA-CA and CA-CB correlations can be detected due to weak C'-CA recoupling ${ }^{19}$.

${ }^{15} \mathrm{~N}-{ }^{13} \mathrm{C}$ : An NCA (total acquisition time: $14 \mathrm{~h}$ ) and an NCACX (total acquisition time: $61 \mathrm{~h}$ ) spectrum were recorded at $800 \mathrm{MHz}$. Contact times were set to $t_{\mathrm{HN}}=600$ $\mu$ s and $t_{\mathrm{NC}}=3 \mathrm{~ms}$, respectively. CACX polarization transfer was established via 
proton-driven spin diffusion for $50 \mathrm{~ms}$. In addition, NCACB (total acquisition time: $19 \mathrm{~h}$ ) and NCOCX (total acquisition time: $85 \mathrm{~h})$ spectra were recorded $(600 \mathrm{MHz})$. Contact times were set to $t_{\mathrm{HN}}=400 \mu \mathrm{s}$ and $t_{\mathrm{NC}}=2 \mathrm{~ms}$, respectively. CC polarization transfer was established via DARR ${ }^{21}$ mixing for $10 \mathrm{~ms}$ (CACB) or $15 \mathrm{~ms}$ (COCX).

For the indirect detection of ${ }^{1} \mathrm{H}-{ }^{1} \mathrm{H}$ correlations, the following experiments were conducted on the $800 \mathrm{MHz}$ instrument:

${ }^{13} \mathrm{C} /{ }^{15} \mathrm{~N}-\mathrm{HH}-{ }^{13} \mathrm{C}$ : Three $\mathrm{CHHC}$ spectra with ${ }^{1} \mathrm{H}-{ }^{1} \mathrm{H}$ mixing times of $250 \mu \mathrm{s}, 325 \mu \mathrm{s}$ and $400 \mu$ s were acquired (37-71 h total acquisition time). Short contact times of $t_{\mathrm{HC}}=100$ $\mu$ s enclosing the ${ }^{1} \mathrm{H}-{ }^{1} \mathrm{H}$ transfer step ensured polarization transfer within bonded ${ }^{1} \mathrm{H}$ ${ }^{13} \mathrm{C}$ pairs only. The contact time of the initial cross-polarization was set to $200 \mu \mathrm{s}$. One NHHC spectrum with a ${ }^{1} \mathrm{H}-{ }^{1} \mathrm{H}$ mixing time of $100 \mu$ s was recorded (46 h total acquisition time). Contact times were set to $t_{\mathrm{HN}}=250 \mu \mathrm{s}$ and $t_{\mathrm{HC}}=100 \mu \mathrm{s}$, respectively.

All spectra were processed using QSINE window functions in F1 and F2 and analyzed using Sparky version 3.106 (T. D. Goddard \& D. G. Kneller, University of California).

\subsection{Resonance assignments and structural constraints}

${ }^{13} \mathrm{C}$ resonances were calibrated using adamantane as an external reference. The upfield resonance of adamantane was set to $31.47 \mathrm{ppm}$ to allow for a direct comparison of the solid-state chemical shifts to solution-state NMR data. Accordingly, ${ }^{15} \mathrm{~N}$ resonances were calibrated using the tripeptide $\mathrm{AGG}^{22}$ as an external reference. Resonance assignments have been deposited in the BMRB data bank (accession number 6351, table 3.2).

${ }^{13} \mathrm{CA},{ }^{13} \mathrm{CB}$ and ${ }^{15} \mathrm{NH}$ chemical shifts served as input parameters for the program TALOS version 2003.027.13.05 23 that predicts the backbone dihedral angles $\phi$ and $\psi$ based on chemical shift and sequence homology with proteins of known structure and assigned (solution-state) chemical shifts. The resulting dihedral angle constraints are 
summarized in Figure 3.3 (top). In the first round of structure calculations, only unambiguous $\mathrm{CHHC}$ constraints were used (table 3.1a). For each possible assignment of ambiguous CHHC correlations, the minimum distance between the involved protons was calculated over the ensemble of preliminary structures. The assignment corresponding to the shortest minimum distance was chosen. If the minimum was found below a threshold of $5.5 \AA$, the corresponding correlation (table 3.1b) was included into a subsequent set of structure calculations.

Table 3.1. ${ }^{1} \mathrm{H}-{ }^{1} \mathrm{H}$ distance constraints.

\begin{tabular}{cclccc}
\hline a) & Res [\#] & & \multicolumn{5}{c}{ Res [\#] } \\
2 & VAL & HB & 21 & ALA & QB* \\
4 & ILE & QD1 & 6 & VAL & QG1 \\
4 & ILE & QG1 & 6 & VAL & QG2 \\
4 & ILE & QG1 & 33 & CYS & QB \\
6 & VAL & QG1 & 17 & PRO & HA \\
8 & CYS & QB & 33 & CYS & HA \\
4 & ILE & QG1 & 35 & CYS & HA \\
\hline b) Res [\#] & & & Res [\#] & & \\
4 & ILE & QD1 & 33 & CYS & HA \\
23 & MET & HA & 37 & PRO & QB \\
3 & GLU & HA & 23 & MET & QB \\
4 & ILE & QD1 & 6 & VAL & HB \\
8 & CYS & HA & 17 & PRO & QD \\
2 & VAL & HA & 37 & PRO & QB \\
36 & THR & HA & 37 & PRO & QD \\
11 & SER & HA & 12 & PRO & QD \\
6 & VAL & QG2 & 17 & PRO & QB \\
6 & VAL & QG2 & 17 & PRO & QG \\
4 & ILE & QG1 & 6 & VAL & HB \\
6 & VAL & HB & 17 & PRO & QB \\
33 & CYS & HA & 34 & HIS & HA \\
35 & CYS & HA & 37 & PRO & QD \\
12 & PRO & HA & 15 & LEU & HG \\
11 & SER & HA & 28 & CYS & HA \\
8 & CYS & HA & 9 & SER & QB \\
10 & GLY & QA & 11 & SER & HA \\
10 & GLY & QA & 12 & PRO & QD \\
6 & VAL & HA & 32 & LYS & HA \\
28 & CYS & QB & 29 & MET & QG \\
\hline${ }^{\star}$ the letter Q indicates methyl or methylene protons & \\
\hline & & & & &
\end{tabular}


Table 3.2. ${ }^{13} \mathrm{C} /{ }^{15} \mathrm{~N}$ chemical shift assignments (ppm).

\begin{tabular}{|c|c|c|c|c|c|c|c|c|}
\hline & C & CA & CB & CG(1) & CG2 & CD(1) & CD2 & N \\
\hline G1 & 169,6 & 43,3 & & & & & & 115,4 \\
\hline V2 & & 60,6 & 34,9 & 20,7 & 20,7 & & & 118,7 \\
\hline E3 & & 56,1 & 33,4 & 35,9 & & & & 126,9 \\
\hline I4 & 175,3 & 59,3 & 41,0 & 26,0 & 17,6 & 13,6 & & 117,3 \\
\hline N5 & & 52,4 & 36,7 & & & & & \\
\hline V6 & 174,5 & 61,5 & 36,7 & 21,9 & 21,2 & & & 121,0 \\
\hline K7 & 174,8 & 55,9 & 33,3 & 25,0 & & 29,4 & & 127,2 \\
\hline C8 & 172,9 & 54,0 & 46,2 & & & & & 114,8 \\
\hline S9 & 173,9 & 57,7 & 64,5 & & & & & 111,7 \\
\hline G10 & 173,9 & 44,5 & & & & & & 108,7 \\
\hline S11 & 173,0 & 61,2 & 64,3 & & & & & 120,3 \\
\hline P12 & 177,3 & 66,1 & 31,2 & 28,6 & & 51,4 & & 136,5 \\
\hline Q13 & & 57,0 & 28,8 & 33,8 & & 180,4 & & 110,9 \\
\hline C14 & & & & & & & & 112,2 \\
\hline L15 & & 58,4 & 41,3 & 27,1 & & 25,1 & 22,7 & 121,8 \\
\hline K16 & & 60,5 & 29,3 & 25,2 & & & & \\
\hline P17 & 176,6 & 66,3 & 31,4 & 28,4 & & 49,7 & & 133,4 \\
\hline C18 & & & & & & & & 110,5 \\
\hline A21 & 175,6 & 51,3 & 18,4 & & & & & 119,6 \\
\hline G22 & 174,1 & 45,5 & & & & & & 105,6 \\
\hline M23 & & 53,9 & 31,2 & & & & & 119,4 \\
\hline R24 & 178,0 & 52,3 & 36,9 & & & & & \\
\hline F25 & & 54,8 & 36,7 & & & & & 120,4 \\
\hline G26 & 174,5 & 45,9 & & & & & & 106,5 \\
\hline K27 & 175,1 & & 33,5 & 25,1 & & 29,3 & & 118,5 \\
\hline C28 & & 54,2 & 37,5 & & & & & \\
\hline M29 & & 55,0 & 35,4 & 31,4 & & & & \\
\hline K32 & & 54,9 & 36,6 & 24,7 & & 28,6 & & \\
\hline C33 & 173,5 & 55,1 & 38,1 & & & & & 120,0 \\
\hline H34 & & 52,2 & & & & & & 118,9 \\
\hline C35 & 54,5 & 33,4 & & & & & 116,9 \\
\hline T36 & 172,0 & 60,2 & 71,0 & & 20,7 & & & \\
\hline P37 & 176,1 & 62,8 & 32,6 & 27,5 & & 51,6 & & \\
\hline
\end{tabular}

\subsection{Molecular-dynamics based structure calculations}

All structure calculations were performed within CNS (Crystallography and NMR System) version $1.1^{24} .{ }^{1} \mathrm{H}-{ }^{1} \mathrm{H}$ distance constraints obtained from CHHC $2 \mathrm{D}$ spectra were represented by square-well potentials with $[1.8,3.5] \AA$ intervals for unambiguous and $[1.8,5] \AA$ intervals for ambiguous constraints, respectively. Ambiguities resulting from restraints involving methyl or methylene protons were 
accounted for during the calculation by using an $\mathrm{r}^{-6}$ summation involving all possible proton-proton contacts ${ }^{25}$. Analogously, dihedral angle constraints derived from TALOS were represented by square-well potentials with upper and lower limits defined by the RMSD of the prediction similar to earlier solution-state NMR studies ${ }^{26}$.

Table 3.3. Structural statistics for 10 final structures.

\begin{tabular}{|c|c|}
\hline \multicolumn{2}{|l|}{ Energies $\left[\mathrm{kcal} \mathrm{mol}{ }^{-1}\right]^{*}$} \\
\hline $\mathrm{E}_{\text {total }}$ & $62 \pm 6$ \\
\hline $\mathrm{E}_{\mathrm{bonds}}$ & $2.0 \pm 0.3$ \\
\hline$E_{\text {angles }}$ & $24 \pm 2$ \\
\hline$E_{\text {impropers }}$ & $4.5 \pm 0.7$ \\
\hline $\mathrm{E}_{\mathrm{van} \text { der Waals }}$ & $21 \pm 2$ \\
\hline $\mathrm{E}_{\mathrm{HH} \text { constraints }}$ & $2.6 \pm 1.0$ \\
\hline $\mathrm{E}_{\text {dihedral angle constraints }}$ & $7.6 \pm 1.4$ \\
\hline Average backbone RMSD to mean & $0.81 \AA$ \\
\hline \multicolumn{2}{|l|}{ Backbone RMSD (residues 4-38) between } \\
\hline mean solid and solution-state (PDB: & $1.9 \AA$ \\
\hline \multicolumn{2}{|l|}{ 2KTX) structures } \\
\hline \multicolumn{2}{|l|}{ Residues in } \\
\hline most favored regions & $80.7 \%$ \\
\hline additional allowed regions & $11.0 \%$ \\
\hline generously allowed regions & $5.0 \%$ \\
\hline disallowed regions & $3.3 \%$ \\
\hline of the Ramachandran plot** & \\
\hline
\end{tabular}

An extended conformer of Kaliotoxin was created as an initial structure and was subsequently subjected to a simulated annealing protocol consisting of three stages: 1 . High temperature annealing in torsion angle space, in 3000 steps of 0.005 ps at 50000 K. 2. Slow-cool annealing stage in torsion angle space, in 3000 steps of 0.005 ps, and temperature reduction from $50000 \mathrm{~K}$ to zero in steps of $250 \mathrm{~K}$. 3. Final energy 
minimization of 2000 steps. During the three stages, force constants $k_{\mathrm{HH}}$ of 300,300 and $150 \mathrm{kcal} \mathrm{mol}^{-1} \AA^{-2}$ and $k_{\text {dihedral }}$ of 100,200 , and $400 \mathrm{kcal} \mathrm{mol}^{-1} \mathrm{rad}^{-2}$, respectively, were used. Likewise, the scale factors for the van-der-Waals energy term were set to $0.1,0.1 \rightarrow 1$ and 1 . An ensemble of 50 structures for the preliminary structure calculations and 200 for the final calculations were generated, starting from different initial velocities. The resulting structures were sorted by total energy, and the 10 lowest energy structures were selected and aligned along the backbone using MOLMOL 2K. $2^{27}$.

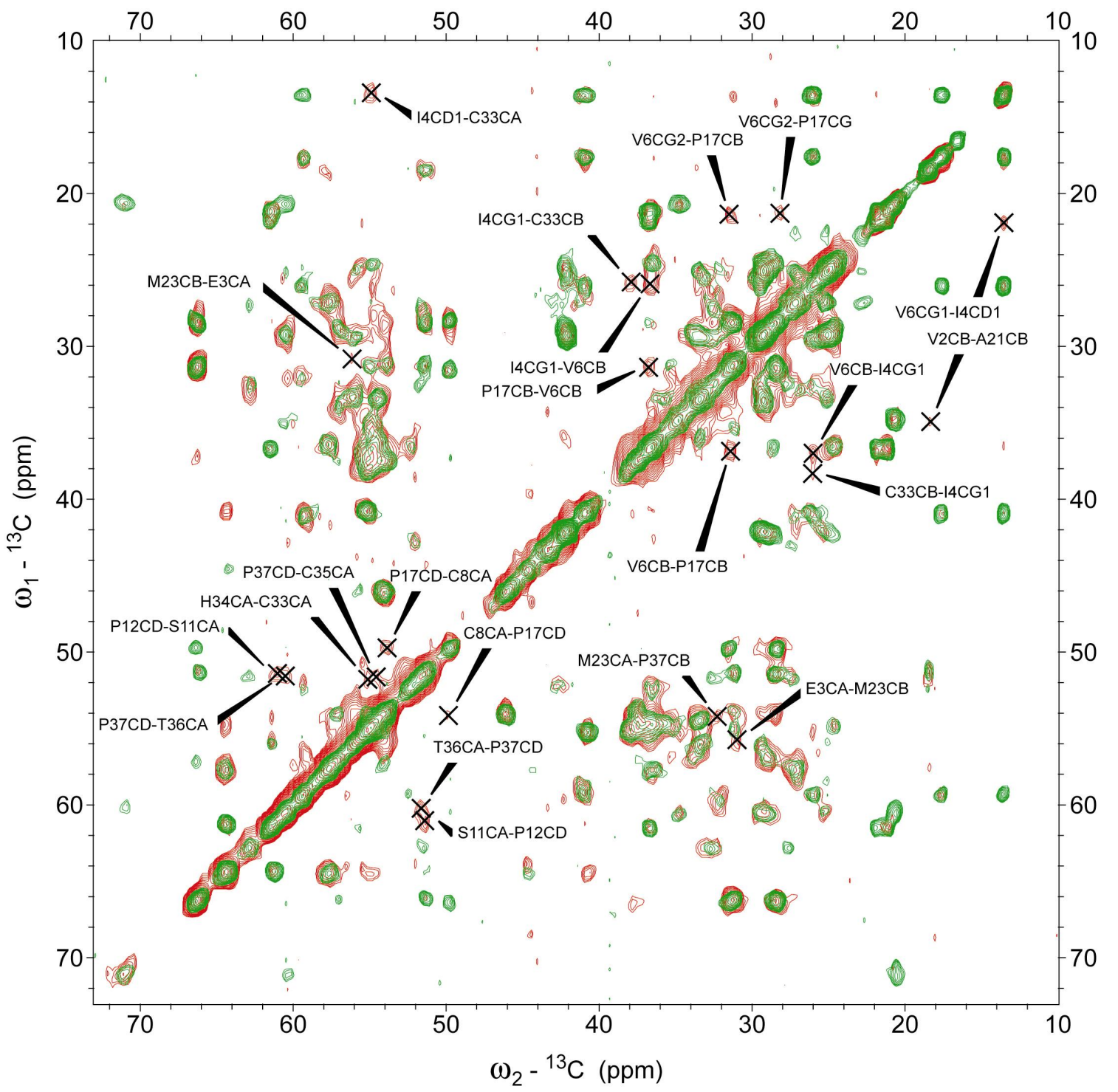

Figure 3.2. Comparison of a $2 \mathrm{D}{ }^{13} \mathrm{C}^{13} \mathrm{C}$ spin-diffusion spectrum (green, mixing time: $50 \mathrm{~ms}$ ) and a CHHC spectrum (red, ${ }^{1} \mathrm{H}-{ }^{1} \mathrm{H}$ mixing time: $250 \mu \mathrm{s}$ ) of $\mathrm{U}-\left[{ }^{13} \mathrm{C},{ }^{15} \mathrm{~N}\right]$ labeled KTX recorded on an 800 $\mathrm{MHz}$ NMR spectrometer (Bruker Biospin). Assigned correlations reflect inter-residue CHHC constraints. 


\subsection{Results}

First, a series of two-dimensional ${ }^{13} \mathrm{C}-{ }^{13} \mathrm{C}$ and ${ }^{15} \mathrm{~N}-{ }^{13} \mathrm{C}$ correlation experiments were conducted on a uniformly $\left[{ }^{13} \mathrm{C},{ }^{15} \mathrm{~N}\right]$ labeled sample of KTX under MAS conditions to establish sequential ${ }^{13} \mathrm{C} /{ }^{15} \mathrm{~N}$ resonance assignments. For example, Figure 3.2 contains the results of a ${ }^{13} \mathrm{C}-{ }^{13} \mathrm{C}$ spin-diffusion experiment ${ }^{28}$ (green), which reveals a variety of intra-residue side chain correlations with typical ${ }^{13} \mathrm{C}$ line widths well below $1 \mathrm{ppm}$. The combination of ${ }^{15} \mathrm{~N}-{ }^{13} \mathrm{C}$ and ${ }^{13} \mathrm{C}-{ }^{13} \mathrm{C} 2 \mathrm{D}$ correlation methods resulted in de novo ${ }^{13} \mathrm{C} /{ }^{15} \mathrm{~N}$ resonance assignments for $87 \%$ of all peptide residues (deposited in the BioMagResBank under accession number 6351, table 3.2). The remaining residues could not be assigned due to spectral overlap.
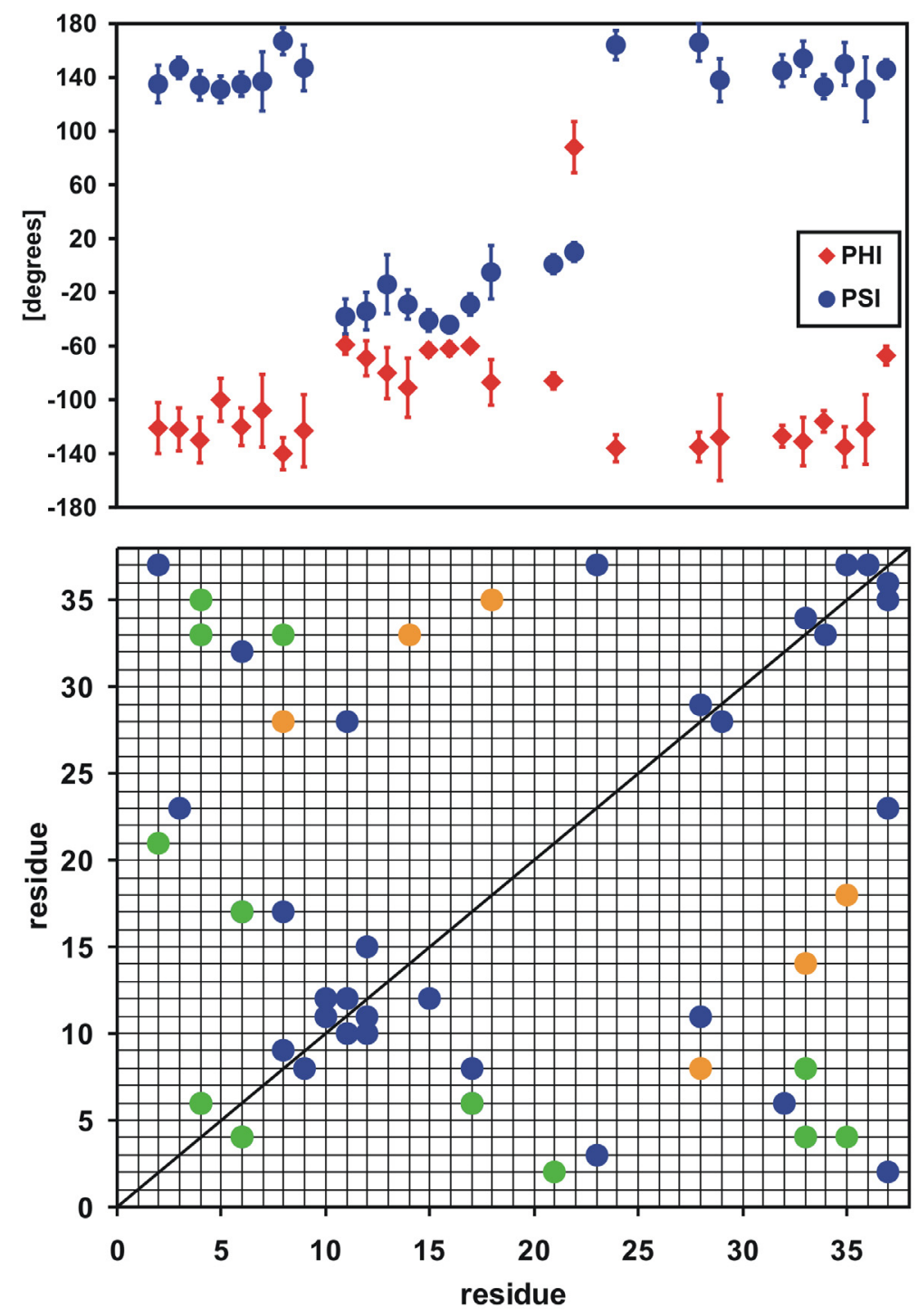

Figure 3.3. Top: Dihedral-angle constraints derived from de novo ${ }^{13} \mathrm{C} /{ }^{15} \mathrm{~N}$ resonance assignments with TALOS. Bottom: Inter-residue distance restraints based on unambiguous (green) and ambiguous (blue) CHHC interactions. Disulfide bonds are depicted in orange. 
Spectral assignments derived from MAS-based solid-state NMR spectroscopic experiments can be analyzed for selectively ${ }^{29}$ or uniformly $^{22}$ isotope-labeled polypeptides with regard to the conformation of the protein backbone by using solution-state NMR spectroscopic data as a reference. Thus, the ${ }^{13} \mathrm{C} /{ }^{15} \mathrm{~N}$ resonance assignments obtained for $\mathrm{U}-\left[{ }^{13} \mathrm{C},{ }^{15} \mathrm{~N}\right]$ labeled KTX (U=uniformly) served as input parameters for the program TALOS ${ }^{23}$ and resulted in a total set of $2 \times 27$ constraints on the dihedral angles of the peptide backbone. As shown in Figure 3.3 (top), the resulting parameters are indicative of $\beta$-strand arrangements comprising residues 2-9, $24,28,29$, and 32-37, and suggest the existence of an $\alpha$-helix containing residues 1121.

Sequential medium- and long-range ${ }^{1} \mathrm{H}-{ }^{1} \mathrm{H}$ distance constraints were determined subsequently from the analysis of three ${ }^{13} \mathrm{C}-{ }^{13} \mathrm{C}$ encoded ${ }^{1} \mathrm{H}-{ }^{1} \mathrm{H}$ mixing experiments $\left(\mathrm{CHHC}^{30,31}\right.$, see also chapter 2$)$. With mixing times of $250-400 \mu \mathrm{s}$ and an MAS rate of $12.5 \mathrm{kHz}\left(B_{0}=18.8 \mathrm{~T}\right)$, the spectra are dominated by ${ }^{1} \mathrm{H}-{ }^{1} \mathrm{H}$ interactions corresponding to distances of $1.8-3.5 \AA$ in a uniformly $\left[{ }^{13} \mathrm{C}\right]$ labeled sample ${ }^{30}$. Figure 3.2 shows the experimental results of a CHHC correlation experiment for a ${ }^{1} \mathrm{H}-{ }^{1} \mathrm{H}$ mixing time of $250 \mu \mathrm{s}$ (red), whereby ${ }^{13} \mathrm{C}-{ }^{13} \mathrm{C}$ cross-peak intensities reflect ${ }^{1} \mathrm{H}-{ }^{1} \mathrm{H}$ interactions. Correlations assigned in Figure 3.2 include long-range contacts along the polypeptide chain and connect protein segments of different secondary structure (Figure 3.3, bottom). Additional distance restraints were deduced from the analysis of CHHC spectra with mixing times of 325 and $400 \mu$ s.

To construct the 3D structure of KTX, solution-state NMR spectroscopic methods ${ }^{32}$ were adapted as follows: In a first round of molecular-dynamics based structure calculations in $\mathrm{CNS}^{24}$, dihedral-angle restraints, as given in Figure 3.3 (top), information about the covalent structure (three disulfide bonds), and eight unambiguously assigned CHHC constraints were used (see table 3.1a). Because ${ }^{1} \mathrm{H}-{ }^{1} \mathrm{H}$ interactions are detected indirectly through the spins of bonded ${ }^{13} \mathrm{C}$ atoms, the assignment of methylene and methyl hydrogen atoms remains ambiguous. Following solution-state NMR spectroscopic approaches ${ }^{25}$, this aspect was accounted for during the calculation by using an $r^{-6}$ summation involving all possible ${ }^{1} \mathrm{H}-{ }^{1} \mathrm{H}$ contacts (see also reference ${ }^{30}$ ). In principle, incorrect or missing assignments and inter-molecular 
interactions can affect the reliability of the CHHC-detected constraints. A systematic analysis reveals that such influences characterize one of the CHHC-detected distance constraints, which leads to a significant increase in overall energy in the structure calculation. This constraint was therefore removed for the later stages of structure refinement. Notably, this CHHC correlation was also not observed for a $\mathrm{U}-\left[{ }^{13} \mathrm{C},{ }^{15} \mathrm{~N}\right]$ labeled sample diluted in unlabeled KTX.

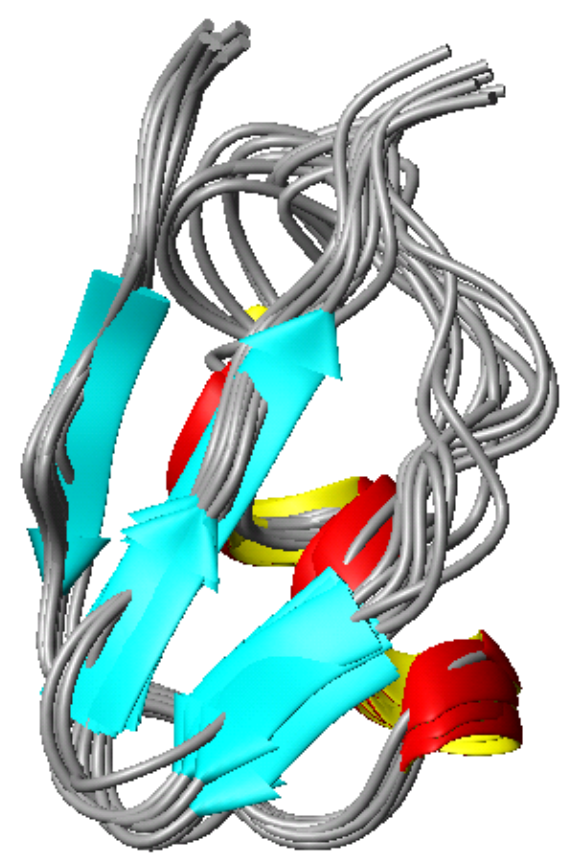

Figure 3.4. Ribbon diagram of the ten conformers determined by solid-state MAS NMR spectroscopy with the lowest energy (PDB entry: 1XSW). The conformers were aligned along the backbone by using MOLMOL ${ }^{27}$.

Next, an ensemble of structures was calculated, which was used to assign ambiguous CHHC correlations (see for example reference ${ }^{32}$ ). Finally, 54 dihedral-angle constraints and 28 distance constraints (6 sequential, 7 medium-range, and 15 longrange) were employed to calculate 200 structures. As in the model study in reference ${ }^{30}$, these ${ }^{1} \mathrm{H}-{ }^{1} \mathrm{H}$ contacts (see table 3.1 ) also involve methyl-methyl interactions. The 10 conformers with the lowest energy (Figure 3.4; PDB entry: 1XSW) exhibit a good covalent structure and a converging fold with an average backbone root-mean-square deviation (RMSD) of $0.81 \AA$ (see table 3.3). Structures with a different fold display significantly higher overall energies (Figure 3.5). 


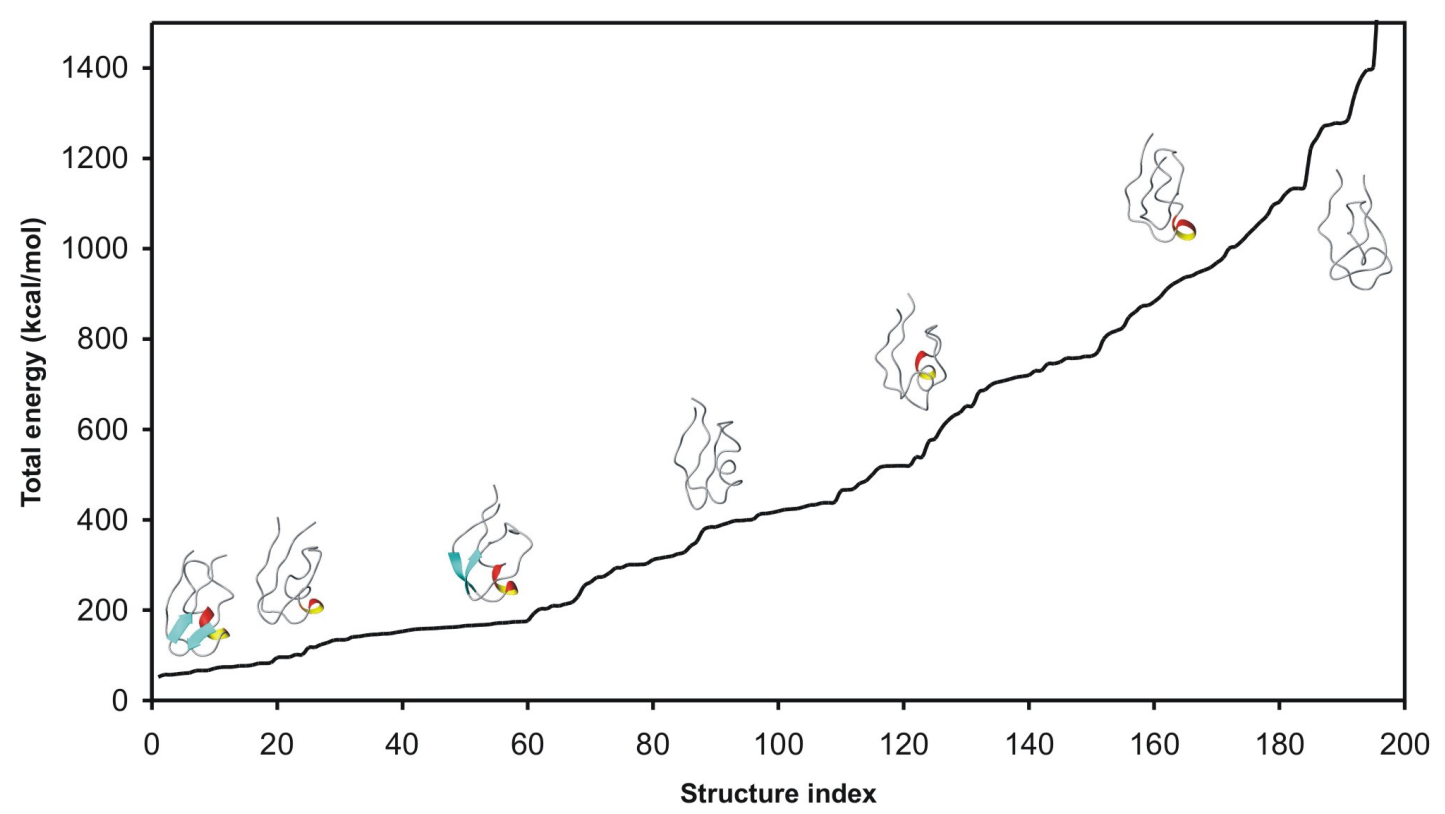

Figure 3.5. Relationship between calculated structures (sorted by total energy), total energy and overall fold for KTX. Structures 2, 20, 55, 90, 125, 160 and 195 are shown.

The backbone RMSD (residues 4-38) between the mean solid-state and solution-state structures (PDB entry: 2KTX based on ${ }^{1} \mathrm{H}$ NMR spectroscopy ${ }^{33}$ ) is $1.9 \AA$. Solutionand solid-state NMR spectroscopic structures are described by the same characteristic $\alpha / \beta$ fold. Larger differences are seen for residues that are not assigned in the solidstate (especially around K19) and for residues in the N-terminal (first) $\beta$-strand. In solution, this $\beta$-strand is partially disorderd. Further evidence for the validity of the derived solid-state NMR spectroscopic structure comes from a PROCHECK NMR analysis, which yields $81 \%$ of all peptide residues in most favored regions. A back calculation of CHHC and additionally recorded $\mathrm{NHHC}^{30} 2 \mathrm{D}$ spectra, which not only provide tertiary contacts but also reflect backbone conformation, confirmed the 3D structure shown in Figure 3.4. These results demonstrate the general validity of the proposed concept.

\subsection{Conclusions}

The determination of the 3D structure of fully or partially immobilized biomolecules, such as membrane or fibrous proteins and their ligands, can be a significant challenge 
for conventional biophysical methods. The use of established solution-state NMR spectroscopic methods for the direct (NOESY-type ${ }^{13}$ ) detection of ${ }^{1} \mathrm{H}-{ }^{1} \mathrm{H}$ contacts in the solid-state is usually prohibited by spectral dispersion and line width. For this reason, ${ }^{13} \mathrm{C} /{ }^{15} \mathrm{~N}$ correlation spectroscopy must generally be used for high-resolution solid-state NMR spectroscopic applications, and the collection of a sufficient number of long-range constraints until now required a high degree of spectral dispersion or the study of differently isotope-labeled samples. With the introduced approach it is possible to construct a $3 \mathrm{D}$ molecular structure from a single uniformly labeled solidphase sample. It relies on the encoding of short ${ }^{1} \mathrm{H}-{ }^{1} \mathrm{H}$ distances in high-resolution ${ }^{13} \mathrm{C} /{ }^{15} \mathrm{~N}$ evolution and detection dimensions and exploits the high sensitivity of chemical shifts detected by solid-state NMR spectroscopy to the conformation of the protein backbone.

In close analogy to NOESY-type experiments on soluble molecules, the general applicability of the concept discussed herein and the accuracy of the derived structures are predominantly determined by spectral resolution. As ${ }^{1} \mathrm{H}-{ }^{1} \mathrm{H}$ correlations are detected indirectly, the sensitivity of the CHHC / NHHC experiments is lower than that of standard ${ }^{13} \mathrm{C} /{ }^{15} \mathrm{~N}$ correlation methods. Unless prohibited by dynamic or structural disorder, the accuracy of the derived structure can be further improved by including intra-residue structural constraints obtained from side chain resonances, by employing three-dimensional NMR spectroscopy ${ }^{34}$, or by improving the structural homogeneity of the sample under study. In the case of KTX, such conditions could be established by preparing microcrystals or by complexing the ligand with KcsA-Kv1.3 potassium channels ${ }^{17}$.

Restriction of the mixing time of the CHHC / NHHC experiments to the initial rate regime not only increases the structural reliability of the detected distance restraints but also reduces spectral overlap. NMR spectroscopic data obtained with longer mixing times can be incorporated into the structural analysis to increase the number of ${ }^{1} \mathrm{H}-{ }^{1} \mathrm{H}$ distance restraints, but at the cost of reduced structural accuracy. In the case of KTX, two-dimensional spectroscopy was sufficient to determine the $3 \mathrm{D}$ conformation by using a set of dihedral-angle constraints, ${ }^{1} \mathrm{H}-{ }^{1} \mathrm{H}$ distance constraints, and three disulfide bridges. Additional structure calculations (without the inclusion of disulfide 
bonds) show that the latter parameters are not critical for the general validity of this approach.

The concept is also amenable to the study of non-peptidic biomolecules, whereby a combination of ${ }^{1} \mathrm{H}-{ }^{1} \mathrm{H}$ and selective ${ }^{13} \mathrm{C}-{ }^{13} \mathrm{C}$ distance restraints may be measured, again for one uniformly labeled sample ${ }^{35}$. As in the case of KTX, which blocks Kv channels with high affinity, ligand / receptor interactions, which are of great pharmacological relevance, may thus be probed readily. Moreover, the technique can be applied to structural studies of membrane proteins and protein fibrils. Depending on the molecule under investigation, the presented approach can be combined with other solid-state NMR spectroscopic methods. For example, in the case of membraneembedded proteins, additional structural constraints could be obtained from a macroscopically aligned specimen. Applications to larger (membrane) proteins may benefit from the use of block ${ }^{11,36}$, modular ${ }^{37}$, reverse $^{38,39}$ isotope labeling.

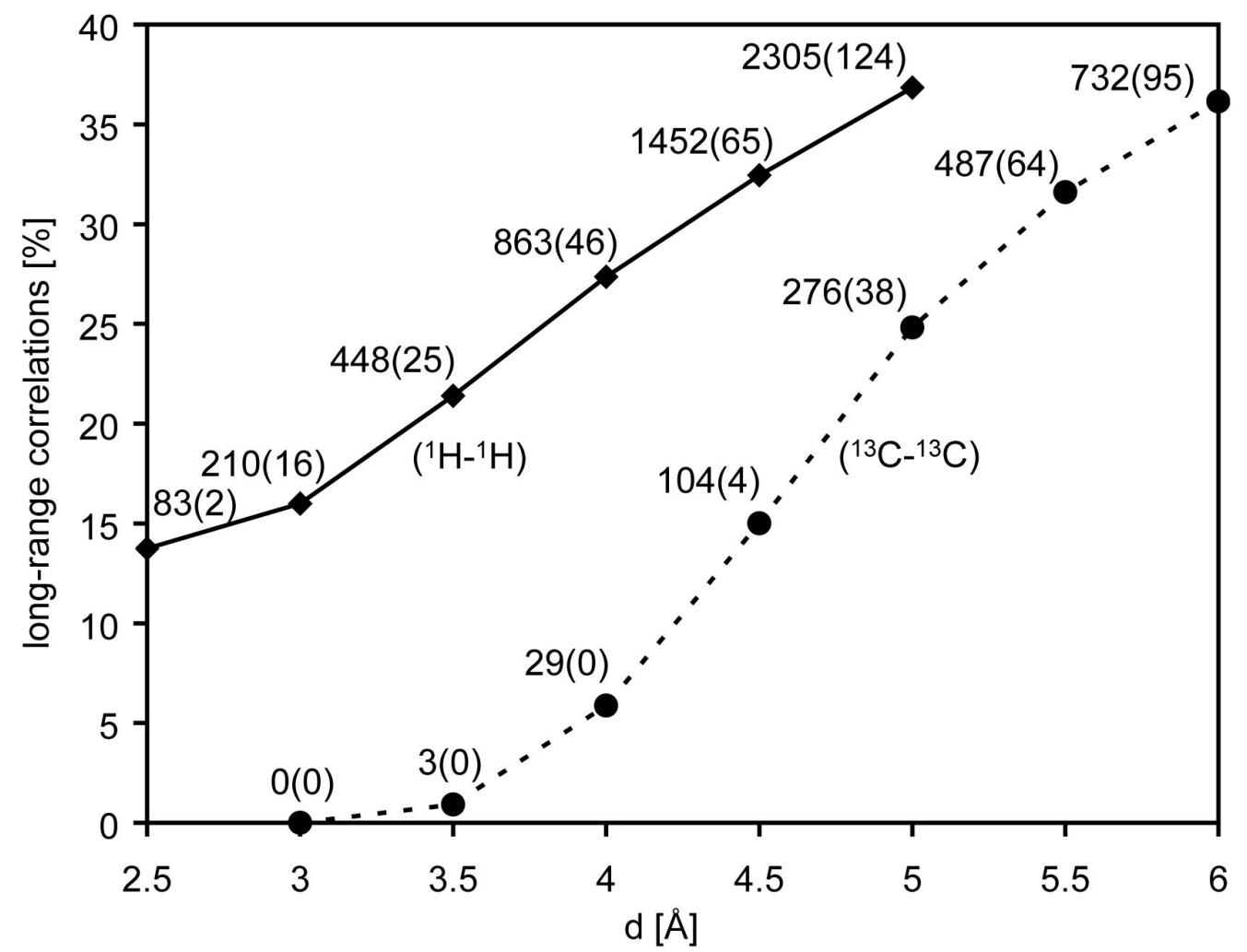

Figure 3.6. For the 76-residue protein ubiquitin (PDB entry: $1 \mathrm{D} 3 \mathrm{Z}^{40}$ ) all ${ }^{1} \mathrm{H}-{ }^{1} \mathrm{H}$ distances up to a distance $d$ were calculated. The fraction of long-range correlations $(|\mathrm{i}-\mathrm{j}|>4$ ) as a function of $d$ (solid line) and their total number (in brackets: without side chains) is shown. Correspondingly, all ${ }^{13} \mathrm{C}-{ }^{13} \mathrm{C}$ distances in both $\left[2-{ }^{13} \mathrm{C}\right]$ glycerol and $\left[1,3-{ }^{13} \mathrm{C}\right]$ glycerol labeled ubiquitin samples up to a distance $d$ were calculated (fraction of long-range correlations: dashed line). 
Even the shortest ${ }^{1} \mathrm{H}-{ }^{1} \mathrm{H}$ distances in proteins contain relevant structural information. To demonstrate this, Figure 3.6 contains an analysis of all ${ }^{1} \mathrm{H}-{ }^{1} \mathrm{H}$ distances up to a distance $d$ in the 76-residue protein ubiquitin (PDB entry: 1D3Z ${ }^{40}$ ). The fraction of long-range correlations (i.e., correlations from residue $i$ to residue $j$ with $|i-j|>4$ ) as a function of $d$ (solid line) and their total number (in brackets: without side chain protons) is shown. A significant fraction ( $15 \%)$ of long-range correlations is already present for the smallest considered distance. In other words, $\sim 15 \%$ of the strongest ${ }^{1} \mathrm{H}-{ }^{1} \mathrm{H}$ dipolar couplings in the system are long-range dipolar couplings. By a restriction of the mixing time of the $\mathrm{CHHC} / \mathrm{NHHC}$ experiments to the initial rate regime (defined by the strongest dipolar couplings of the system) this fraction can be detected with high accuracy.

In a uniformly $\left[{ }^{13} \mathrm{C}\right]$ labeled protein the presence of one- and two-bond ${ }^{13} \mathrm{C}-{ }^{13} \mathrm{C}$ dipolar couplings leads to dipolar truncation effects that prevent the detection of longrange ${ }^{13} \mathrm{C}-{ }^{13} \mathrm{C}$ correlations. It has recently been demonstrated that these ${ }^{13} \mathrm{C}-{ }^{13} \mathrm{C}$ longrange correlations can be observed with ${ }^{13} \mathrm{C}-{ }^{13} \mathrm{C}$ experiments in site-directed labeled samples obtained from Escherichia coli cell cultures grown in a medium supplemented with either $\left[2-{ }^{13} \mathrm{C}\right]$ glycerol or $\left[1,3-{ }^{13} \mathrm{C}\right]$ glycerol ${ }^{11}$. In the resulting labeling pattern only few one-bond ${ }^{13} \mathrm{C}-{ }^{13} \mathrm{C}$ dipolar couplings are present and the observation of long-range ${ }^{13} \mathrm{C}-{ }^{13} \mathrm{C}$ correlations becomes feasible. Figure 3.6 contains an analysis of short ${ }^{13} \mathrm{C}-{ }^{13} \mathrm{C}$ distances (up to a distance $d$ ) in both $\left[2-{ }^{13} \mathrm{C}\right]$ glycerol and $\left[1,3-{ }^{13} \mathrm{C}\right]$ glycerol labeled ubiquitin. The fraction of long-range correlations as a function of $d$ (dashed line) and their total number (in brackets: without side chain carbons) is shown. Here, a significant fraction $(\sim 15 \%)$ of long-range correlations is only present if distances up to $4.5 \AA$ are considered. In other words, the strongest ${ }^{13} \mathrm{C}$ ${ }^{13} \mathrm{C}$ dipolar couplings in the system are not long-range dipolar couplings. Therefore, the mixing time in the ${ }^{13} \mathrm{C}-{ }^{13} \mathrm{C}$ experiments can not be restricted to the initial rate regime if long-range correlations are to be observed. As a result, the structural accuracy of the detected distance constraints is compromised and wider constraint classes have to be used in the structure calculations. 
1. Cross, T. A. \& Opella, S. J. Solid-state NMR structural studies of peptides and proteins in membranes. Current Opinion in Structural Biology 4, 574-581 (1994).

2. Griffin, R. G. Dipolar recoupling in MAS spectra of biological solids. Nature Structural Biology 5, 508-512 (1998).

3. Luca, S., Heise, H., Lange, A. \& Baldus, M. Investigation of ligand-receptor systems by high-resolution solid-state NMR: Recent progress and perspectives. Archiv der Pharmazie 338, 217-228 (2005).

4. McDermott, A. E. Structural and dynamic studies of proteins by solid-state NMR spectroscopy: Rapid movement forward. Current Opinion in Structural Biology 14, 554-561 (2004).

5. Opella, S. J. \& Marassi, F. M. Structure determination of membrane proteins by NMR spectroscopy. Chemical Reviews 104, 3587-3606 (2004).

6. Andronesi, O. C. et al. Determination of membrane protein structure and dynamics by magic-angle-spinning solid-state NMR spectroscopy. Journal of the American Chemical Society 127, 12965-12974 (2005).

7. Nomura, K., Takegoshi, K., Terao, T., Uchida, K. \& Kainosho, M. Determination of the complete structure of a uniformly labeled molecule by rotational resonance solid-state NMR in the tilted rotating frame. Journal of the American Chemical Society 121, 4064-4065 (1999).

8. Rienstra, C. M. et al. De novo determination of peptide structure with solidstate magic-angle-spinning NMR spectroscopy. Proceedings of the National Academy of Sciences of the United States of America 99, 10260-10265 (2002).

9. Petkova, A. T. et al. A structural model for Alzheimer's $\beta$-amyloid fibrils based on experimental constraints from solid-state NMR. Proceedings of the National Academy of Sciences of the United States of America 99, 1674216747 (2002).

10. Jaroniec, C. P. et al. High-resolution molecular structure of a peptide in an amyloid fibril determined by magic-angle-spinning NMR spectroscopy. Proceedings of the National Academy of Sciences of the United States of America 101, 711-716 (2004).

11. Castellani, F. et al. Structure of a protein determined by solid-state magicangle-spinning NMR spectroscopy. Nature 420, 98-102 (2002).

12. Andrew, E. R., Bradbury, A. \& Eades, R. G. Nuclear magnetic resonance spectra from a crystal rotated at high speed. Nature 182, 1659-1659 (1958).

13. Wüthrich, K. NMR of proteins and nucleic acids (Wiley Interscience, New York, 1986).

14. Crest, M. et al. Kaliotoxin, a novel peptidyl inhibitor of neuronal BK-type $\mathrm{Ca}^{2+}$-activated $\mathrm{K}^{+}$channels characterized from Androctonus mauretanicus mauretanicus venom. Journal of Biological Chemistry 267, 1640-1647 (1992).

15. Grissmer, S. et al. Pharmacological characterization of five cloned voltagegated $\mathrm{K}^{+}$channels, types Kv1.1, Kv1.2, Kv1.3, Kv1.5, and Kv3.1, stably expressed in mammalian cell lines. Molecular Pharmacology 45, 1227-1234 (1994).

16. Chong, S. R. et al. Single-column purification of free recombinant proteins using a self-cleavable affinity tag derived from a protein splicing element. Gene 192, 271-281 (1997).

17. Legros, C. et al. Generating a high-affinity scorpion toxin receptor in KcsAKv1.3 chimeric potassium channels. Journal of Biological Chemistry 275, 16918-16924 (2000). 
18. Fung, B. M., Khitrin, A. K. \& Ermolaev, K. An improved broadband decoupling sequence for liquid crystals and solids. Journal of Magnetic Resonance 142, 97-101 (2000).

19. Seidel, K. et al. Protein solid-state NMR resonance assignments from ${ }^{13} \mathrm{C}-{ }^{13} \mathrm{C}$ correlation spectroscopy. Physical Chemistry Chemical Physics 6, 5090-5093 (2004).

20. Kristiansen, P. E., Mitchell, D. J. \& Evans, J. N. S. Double-quantum dipolar recoupling at high magic-angle-spinning rates. Journal of Magnetic Resonance 157, 253-266 (2002).

21. Takegoshi, K., Nakamura, S. \& Terao, T. ${ }^{13} \mathrm{C}-{ }^{1} \mathrm{H}$ dipolar-assisted rotational resonance in magic-angle-spinning NMR. Chemical Physics Letters 344, 631637 (2001).

22. Luca, S. et al. Secondary chemical shifts in immobilized peptides and proteins: A qualitative basis for structure refinement under magic-angle-spinning. Journal of Biomolecular NMR 20, 325-331 (2001).

23. Cornilescu, G., Delaglio, F. \& Bax, A. Protein backbone angle restraints from searching a database for chemical shift and sequence homology. Journal of Biomolecular NMR 13, 289-302 (1999).

24. Brunger, A. T. et al. Crystallography \& NMR system: A new software suite for macromolecular structure determination. Acta Crystallographica Section D-Biological Crystallography 54, 905-921 (1998).

25. Fletcher, C. M., Jones, D. N. M., Diamond, R. \& Neuhaus, D. Treatment of NOE constraints involving equivalent or non-stereoassigned protons in calculations of biomacromolecular structures. Journal of Biomolecular NMR 8, 292-310 (1996).

26. Bonvin, A., Houben, K., Guenneugues, M., Kaptein, R. \& Boelens, R. Rapid protein fold determination using secondary chemical shifts and cross-hydrogen bond ${ }^{15} \mathrm{~N}-{ }^{13} \mathrm{C}^{\prime}$ scalar couplings $\left({ }^{3 \mathrm{hb}} \mathrm{J}_{\mathrm{NC}}\right)$. Journal of Biomolecular NMR 21, 221-233 (2001).

27. Koradi, R., Billeter, M. \& Wüthrich, K. MOLMOL: A program for display and analysis of macromolecular structures. Journal of Molecular Graphics 14, 51-55 (1996).

28. Bloembergen, N. On the interaction of nuclear spins in a crystalline lattice. Physica 15, 386-426 (1949).

29. Saito, H. Conformation-dependent ${ }^{13} \mathrm{C}$ chemical shifts - A new means of conformational characterization as obtained by high-resolution solid-state ${ }^{13} \mathrm{C}$ NMR. Magnetic Resonance in Chemistry 24, 835-852 (1986).

30. Lange, A., Seidel, K., Verdier, L., Luca, S. \& Baldus, M. Analysis of protonproton transfer dynamics in rotating solids and their use for 3D structure determination. Journal of the American Chemical Society 125, 12640-12648 (2003).

31. Lange, A., Luca, S. \& Baldus, M. Structural constraints from proton-mediated rare-spin correlation spectroscopy in rotating solids. Journal of the American Chemical Society 124, 9704-9705 (2002).

32. Nilges, M. Structure calculation from NMR data. Current Opinion in Structural Biology 6, 617-623 (1996).

33. Gairi, M. et al. 3D structure of kaliotoxin: Is residue 34 a key for channel selectivity? Journal of Peptide Science 3, 314-319 (1997).

34. Heise, H., Seidel, K., Etzkorn, M., Becker, S. \& Baldus, M. 3D NMR spectroscopy for resonance assignment and structure elucidation of proteins 
under MAS: Novel pulse schemes and sensitivity considerations. Journal of Magnetic Resonance 173, 64-74 (2005).

35. Seidel, K. et al. Studying molecular 3D structure and dynamics by highresolution solid-state NMR: Application to L-tyrosine-ethylester. Journal of Physical Chemistry A 109, 2436-2442 (2005).

36. LeMaster, D. M. \& Kushlan, D. M. Dynamical mapping of E. coli thioredoxin via ${ }^{13} \mathrm{C}$ NMR relaxation analysis. Journal of the American Chemical Society 118, 9255-9264 (1996).

37. Pickford, A. R. \& Campbell, I. D. NMR studies of modular protein structures and their interactions. Chemical Reviews 104, 3557-3565 (2004).

38. Vuister, G. W., Kim, S. J., Wu, C. \& Bax, A. 2D and 3D NMR study of phenylalanine residues in proteins by reverse isotopic labeling. Journal of the American Chemical Society 116, 9206-9210 (1994).

39. Heise, H. et al. Molecular-level secondary structure, polymorphism, and dynamics of full-length $\alpha$-synuclein fibrils studied by solid-state NMR. Proceedings of the National Academy of Sciences of the United States of America 102, 15871-15876 (2005).

40. Cornilescu, G., Marquardt, J. L., Ottiger, M. \& Bax, A. Validation of protein structure from anisotropic carbonyl chemical shifts in a dilute liquid crystalline phase. Journal of the American Chemical Society 120, 6836-6837 (1998). 


\section{The 3D structure of epothilone B}

\subsection{Introduction}

Epothilone $\mathrm{A}$ and $\mathrm{B}$ are natural compounds produced by the myxobacterium Sorangium cellulosum ${ }^{1,2}$. They are known to stabilize microtubules, the polymerized form of the heterodimeric protein $\alpha \beta$-tubulin ${ }^{3}$. The extensive polymerization of $\alpha \beta$ tubulin induced by epothilones triggers apoptosis (programmed cell death). Because epothilones exhibit a high cytotoxic activity against multiresistant tumor cells $\mathrm{s}^{2-4}$, there is a strong interest in understanding the mechanism of their binding to $\alpha \beta$-tubulin. The crystal structure of epothilone B (see Figure 4.1) is known ${ }^{1}$. However, its bioactive conformation in complex with microtubules remains elusive ${ }^{5}$. Recently a solution-state NMR structure of epothilone A (that lacks the methyl group at C12) in the presence of unpolymerized $\alpha \beta$-tubulin was determined ${ }^{6}$. In addition, an electroncrystallographic study on $\mathrm{Zn}^{2+}$-stabilized $\alpha \beta$-tubulin layers in the presence of epothilone A was performed ${ }^{7}$. While the investigation of the complex of epothilones with polymerized $\alpha \beta$-tubulin, i.e. microtubules, is challenging for solution-state NMR spectroscopy and crystallographic techniques, solid-state NMR experiments on labeled epothilone in complex with non-labeled microtubules could in principle be used to elucidate its bioactive conformation.

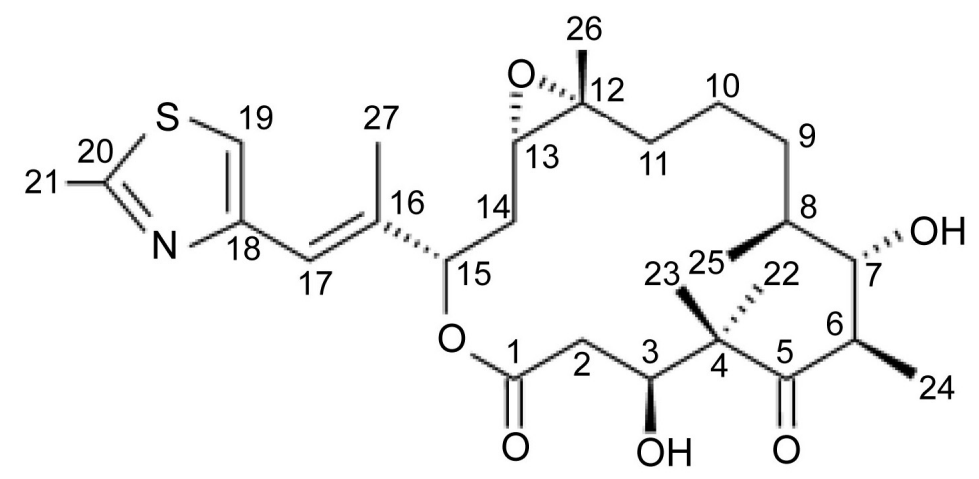

Figure 4.1. The natural product epothilone B by the myxobacterium Sorangium cellulosum. 
To decide which solid-state NMR experiments are most appropriate for obtaining the 3D structure of epothilone B in complex with microtubules the following model study on free, i.e. unbound, epothilone $\mathrm{B}$ was performed. The structure determination approach described in chapter 3 relies on $\mathrm{CHHC} / \mathrm{NHHC}^{8-10}$ derived distance constraints on the one hand and dihedral angle constraints determined by the program TALOS $^{11}$ on the other hand. While the CHHC / NHHC experiments can be applied to arbitrary $\left[{ }^{13} \mathrm{C},{ }^{15} \mathrm{~N}\right]$ labeled molecules, TALOS is only applicable to polypeptides. Therefore, in this chapter it was tested whether CHHC / NHHC data alone are sufficient to determine the $3 \mathrm{D}$ fold of epothilone B. A similar study has previously been performed on L-tyrosine-ethylester ${ }^{12}$.

\subsection{Solid-state NMR spectroscopy}

$\left[{ }^{13} \mathrm{C}\right]$ labeled epothilone was obtained by shake flask fermentation with the myxobacterium Sorangium cellulosum So ce90 by using a high-epothilone-producing mutant, BCE99/41 (Novartis strain collection). The fermentation was performed by growing the strain in a medium $(1000 \mathrm{ml})$ containing $\left[{ }^{13} \mathrm{C}\right]$ labeled $\operatorname{starch}\left({ }^{13} \mathrm{C}_{6}, 98 \%\right.$; $20 \mathrm{~g}$ ) as the carbohydrate source. $20 \mathrm{mg}$ of epothilone A and $15 \mathrm{mg}$ of epothilone B were obtained with an incorporation rate of $60-70 \%{ }^{13} \mathrm{C} .3 \mathrm{mg}$ of $\left[{ }^{13} \mathrm{C}\right]$ labeled epothilone B and $18 \mathrm{mg}$ of unlabeled epothilone B were dissolved in dichloromethane and recrystallized.

Two-dimensional NMR experiments were conducted on a $14.1 \mathrm{~T}\left({ }^{1} \mathrm{H}\right.$ resonance frequency: $600 \mathrm{MHz}$ ) wide-bore instrument (Bruker Biospin, Germany) equipped with a $4 \mathrm{~mm}$ triple-resonance $\left({ }^{1} \mathrm{H},{ }^{13} \mathrm{C},{ }^{15} \mathrm{~N}\right)$ MAS probe. All experiments were carried out at probe temperatures between $-5{ }^{\circ} \mathrm{C}$ and $-8{ }^{\circ} \mathrm{C}$. High power proton-decoupling using the sequences TPPM $^{13}$ or SPINAL64 ${ }^{14}$ with r.f. amplitudes of $80-90 \mathrm{kHz}$ was applied during evolution and detection periods. All spectra were processed using QSINE window functions in F1 and F2 and analyzed using Sparky version 3.111 (T. D. Goddard \& D. G. Kneller, University of California). 
For the assignment of ${ }^{13} \mathrm{C}$ resonances a conventional proton-driven spin diffusion (SD) scheme employing a longitudinal mixing time of $10 \mathrm{~ms}$ was used (MAS frequency: $11.9 \mathrm{kHz}$ ). The contact time $t_{\mathrm{HC}}$ of the ramped cross-polarization was set to $1 \mathrm{~ms}$.

For the indirect detection of ${ }^{1} \mathrm{H}-{ }^{1} \mathrm{H}$ correlations two CHHC spectra with ${ }^{1} \mathrm{H}-{ }^{1} \mathrm{H}$ mixing times of $100 \mu$ s and $150 \mu$ s were acquired (MAS frequency: $11 \mathrm{kHz}$ ). Short contact times of $t_{\mathrm{HC}}=100-150 \mu$ s enclosing the ${ }^{1} \mathrm{H}-{ }^{1} \mathrm{H}$ transfer step ensured polarization transfer within bonded ${ }^{1} \mathrm{H}-{ }^{13} \mathrm{C}$ pairs only. The contact time of the initial crosspolarization was set to $1 \mathrm{~ms}$.

Table 4.1. ${ }^{13} \mathrm{C}$ chemical shift assignments.

\begin{tabular}{|c|c|c|c|}
\hline & $\begin{array}{c}\text { Solid- } \\
\text { state }\end{array}$ & $\begin{array}{c}\text { Liquid- } \\
\text { state }^{1}\end{array}$ & \\
\hline spin & $\delta[$ ppm] & $\delta[p p m]$ & $\Delta[\mathrm{ppm}]$ \\
\hline C1 & 172.7 & 172.1 & 0.6 \\
\hline C2 & 39.5 & 40.2 & -0.7 \\
\hline C3 & 77.5 & 72.5 & 5.0 \\
\hline C4 & 55.7 & 55.2 & 0.5 \\
\hline C5 & 222.7 & 219.4 & 3.3 \\
\hline C6 & 51.1 & 46.9 & 4.2 \\
\hline C7 & 81.8 & 77.5 & 4.3 \\
\hline C8 & 37.5 & 37.6 & -0.1 \\
\hline C9 & 36.3 & 31.6 & 4.7 \\
\hline C10 & 26.8 & 25.0 & 1.8 \\
\hline C11 & 34.9 & 34.1 & 0.8 \\
\hline C12 & 65.6 & 63.0 & 2.6 \\
\hline C13 & 67.1 & 63.5 & 3.6 \\
\hline C14 & 35.6 & 35.0 & 0.6 \\
\hline C15 & 80.8 & 78.6 & 2.2 \\
\hline C16 & 139.4 & 139.2 & 0.2 \\
\hline C17 & 124.5 & 121.2 & 3.3 \\
\hline C18 & 155.5 & 154.1 & 1.4 \\
\hline C19 & 121.4 & 119.7 & 1.7 \\
\hline C20 & 168.5 & 166.3 & 2.2 \\
\hline C21 & 23.3 & 20.9 & 2.4 \\
\hline C22 & 23.5 & 21.7 & 1.8 \\
\hline C23 & 26.1 & 24.5 & 1.6 \\
\hline C24 & 19.5 & 18.4 & 1.1 \\
\hline C25 & 22.2 & 20.4 & 1.8 \\
\hline C26 & 26.1 & 24.1 & 2.0 \\
\hline C27 & 15.2 & 16.1 & -0.9 \\
\hline & & & \\
\hline
\end{tabular}




\subsection{Resonance assignments and structural constraints}

${ }^{13} \mathrm{C}$ resonances were calibrated using adamantane as an external reference. The upfield resonance of adamantane was set to $31.47 \mathrm{ppm}$ to allow for a direct comparison of the solid-state chemical shifts to solution-state NMR data. The assigned solid-state chemical shifts are given in table 4.1 (for the numbering of the ${ }^{13} \mathrm{C}$ nuclei see Figure 4.1). For reference, table 4.1 also contains solution-state chemical shift assignments for epothilone $\mathrm{B}$ in $\left[\mathrm{D}_{6}\right] \mathrm{DMSO}^{1}$.

Table 4.2. ${ }^{1} \mathrm{H}-{ }^{1} \mathrm{H}$ distance constraints.

\begin{tabular}{|c|c|c|c|}
\hline a) & $\mathrm{C}[\#]$ & $\mathrm{C}[\#]$ & Volume \\
\hline & 7 & 8 & 100.0 \\
\hline & 9 & 11 & 87.8 \\
\hline & 9 & 10 & 84.5 \\
\hline & 2 & 6 & 79.3 \\
\hline & 13 & 15 & 71.0 \\
\hline & 10 & 11 & 57.7 \\
\hline & 7 & 25 & 55.8 \\
\hline & 2 & 22 & 50.6 \\
\hline & 6 & 9 & 50.6 \\
\hline & 2 & 9 & 42.0 \\
\hline & 6 & 24 & 41.4 \\
\hline & 8 & 9 & 36.7 \\
\hline & 8 & 10 & 36.5 \\
\hline & 13 & 14 & 34.3 \\
\hline & 3 & 23 & 31.8 \\
\hline & 8 & 25 & 28.5 \\
\hline & 9 & 25 & 23.4 \\
\hline & 7 & 24 & 18.7 \\
\hline & 10 & 25 & 18.3 \\
\hline & 2 & 3 & 17.4 \\
\hline & 6 & 8 & 17.0 \\
\hline & 3 & 22 & 15.9 \\
\hline & 14 & 15 & 15.5 \\
\hline & 6 & 7 & 10.6 \\
\hline & 22 & 24 & 10.4 \\
\hline & 6 & 22 & 10.4 \\
\hline & 22 & 23 & 8.9 \\
\hline \multirow[t]{4}{*}{ b) } & $C[\#]$ & $\mathrm{C}[\#]$ & \\
\hline & 15 & 17 & \\
\hline & 17 & 19 & \\
\hline & 2 & 8 & \\
\hline
\end{tabular}


All detected CHHC cross-peaks could be assigned unambiguously. Cross-peaks from the spectrum with a ${ }^{1} \mathrm{H}-{ }^{1} \mathrm{H}$ mixing time of $100 \mu$ s were integrated and sorted by peak volume (see table 4.2a). In addition, correlations from the $150 \mu \mathrm{s}$ CHHC spectrum that were not visible in the $100 \mu$ s CHHC spectrum were considered (see table 4.2b).

\subsection{Molecular-dynamics based structure calculations}

Structure calculations were performed within CNS (Crystallography and NMR System) version $1.1^{15} \cdot{ }^{1} \mathrm{H}-{ }^{1} \mathrm{H}$ distance constraints obtained from CHHC $2 \mathrm{D}$ spectra were represented by square-well potentials. An extended conformer of epothilone B was created as an initial structure and was subsequently subjected to a simulated annealing protocol consisting of three stages: 1. High temperature annealing in cartesian space, in 5000 steps of 0.003 ps at 2000 K. 2. Slow-cool annealing stage in cartesian space, in 5000 steps of $0.005 \mathrm{ps}$, and temperature reduction from $2000 \mathrm{~K}$ to zero in steps of 25 K. 3. Final energy minimization of 2000 steps. During the three stages, force constants $k_{\mathrm{HH}}$ of 50,50 and $25 \mathrm{kcal} \mathrm{mol}^{-1} \AA^{-2}$ were used. Likewise, the scale factors for the van-der-Waals energy term were set to $0.1,0.1 \rightarrow 4$ and 1 . An ensemble of 200 structures was generated, starting from different initial velocities. The resulting structures were sorted by total energy, and the 10 lowest energy structures were selected and aligned along the heavy atoms using MOLMOL $2 \mathrm{~K} .2^{16}$.

\subsection{Results}

First, a ${ }^{13} \mathrm{C}$ proton-driven spin diffusion spectrum with a mixing time of $10 \mathrm{~ms}$ was recorded (Figure 4.2). At this mixing time primarily one-bond correlations are detected. The observed ${ }^{13} \mathrm{C}$ line widths are well below $1 \mathrm{ppm}$. This indicates that the investigated epothilone B sample consists of nano- or microcrystalline powder. In combination with the large dispersion of the resonances (ranging from 15.2 to 222.7 ppm) the resulting high resolution of the spectrum makes the unambiguous assignment of all $27{ }^{13} \mathrm{C}$ resonances (see table 4.1) feasible. The stereospecific assignment of $\mathrm{C} 22$ and $\mathrm{C} 23$ was obtained by CHHC correlations (see below). 


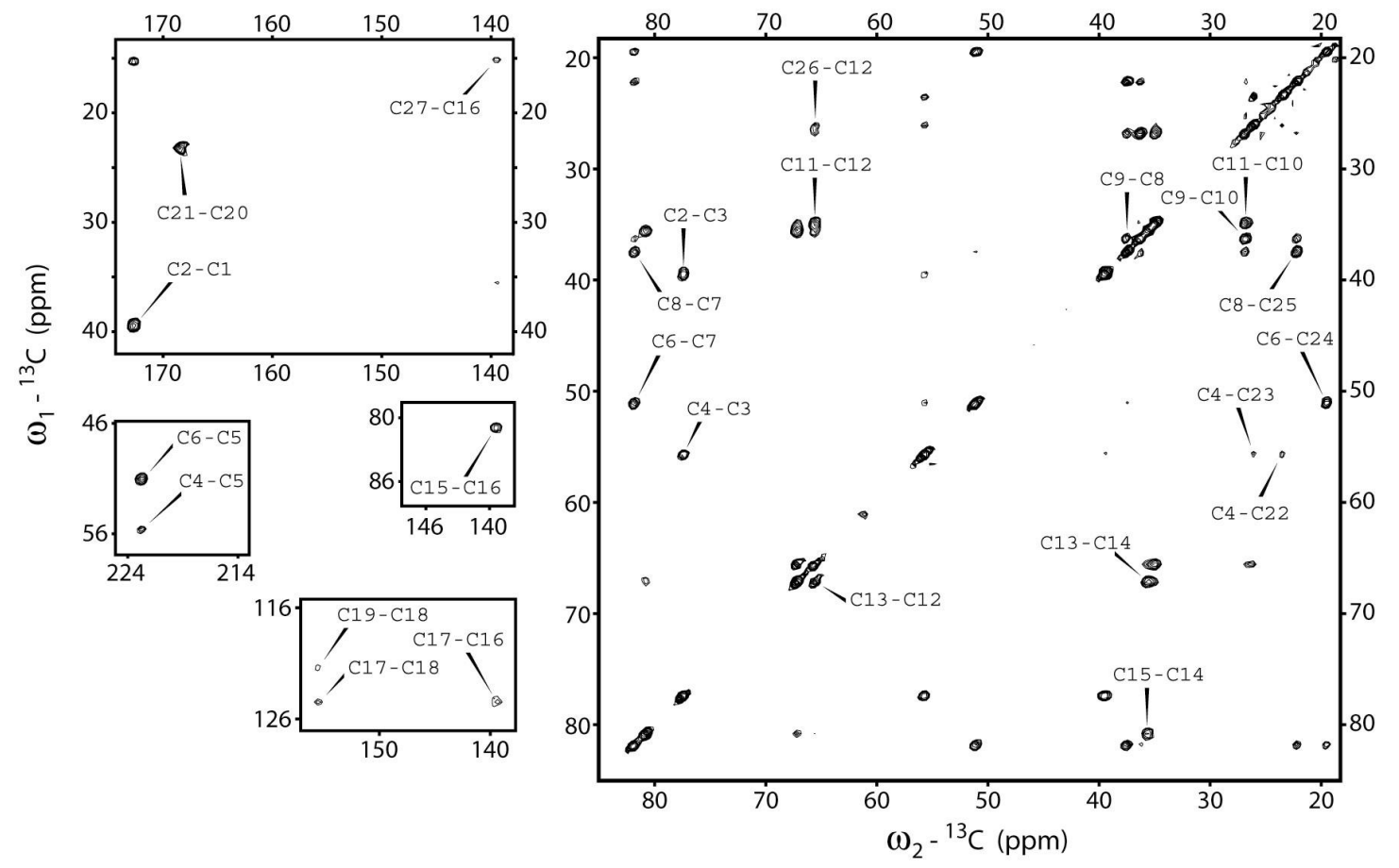

Figure 4.2. $2 \mathrm{D}{ }^{13} \mathrm{C}$ spin-diffusion spectrum (mixing time: $10 \mathrm{~ms}$ ) of $\left[{ }^{13} \mathrm{C}\right]$ labeled epothilone $\mathrm{B}$ recorded on a $600 \mathrm{MHz} \mathrm{NMR}$ spectrometer (Bruker Biospin) at $11.9 \mathrm{kHz}$ MAS. At this mixing time mostly one-bond correlations are observed.

Subsequently, two CHHC spectra with ${ }^{1} \mathrm{H}-{ }^{1} \mathrm{H}$ mixing times of $100 \mu$ s (see Figure 4.3) and $150 \mu$ s were recorded. As described in detail in chapter 2, at these mixing times and at an MAS rate of $11 \mathrm{kHz}\left(B_{0}=14.1 \mathrm{~T}\right)$, CHHC spectra are dominated by ${ }^{1} \mathrm{H}-{ }^{1} \mathrm{H}$ interactions corresponding to distances of 1.8-3.0 $\AA$. Unlike the study described in chapter 3 where spectral overlap and multiple assignment possibilities complicated the spectral analysis, all CHHC cross-peaks are here well-resolved and can be assigned unambiguously. Because the $\left[{ }^{13} \mathrm{C}\right]$ labeled epothilone $\mathrm{B}$ was diluted in natural abundance material, all CHHC cross-peaks result from intra-molecular polarization transfer. CHHC spectra of undiluted small organic molecules contain a large fraction of inter-molecular cross-peaks due to their small ratio of volume and surface. Therefore, isotopic dilution is crucial if a 3D structure determination based on CHHC correlations is to be performed. The problem of inter-molecular cross-peaks could also be overcome by binding epothilone $\mathrm{B}$ to microtubules. In this case the epothilone B binding pockets are well separated in space. 


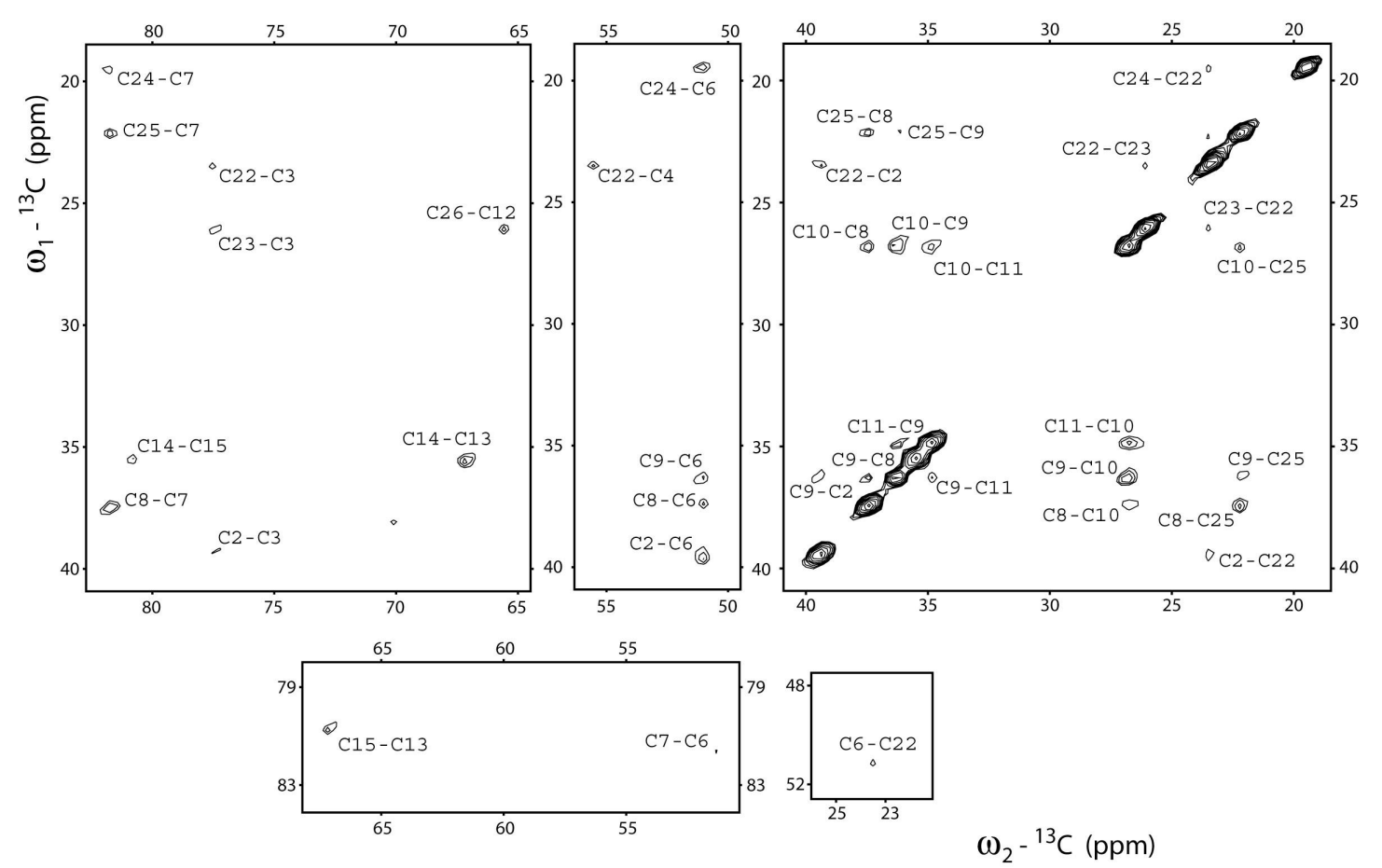

Figure 4.3. $2 \mathrm{D}$ CHHC spectrum $\left({ }^{1} \mathrm{H}-{ }^{1} \mathrm{H}\right.$ mixing time: $100 \mu$ s) of $\left[{ }^{13} \mathrm{C}\right]$ labeled epothilone B recorded on a $600 \mathrm{MHz}$ NMR spectrometer (Bruker Biospin) at $11 \mathrm{kHz}$ MAS. The assigned correlations reflect intra-molecular through-space proton-proton connectivities.

To construct the 3D structure of epothilone $\mathrm{B}$, molecular-dynamics based structure calculations in $\mathrm{CNS}^{15}$ were performed. ${ }^{1} \mathrm{H}-{ }^{1} \mathrm{H}$ distance constraints obtained from CHHC 2D spectra were represented by square-well potentials (see table 4.2). [1.8, 2.5] $\AA$ intervals were used for strong cross-peaks (i.e., 100-50 \% peak volume compared to the strongest cross-peak) in the $100 \mu$ s CHHC spectrum. For weak crosspeaks and those appearing only at a mixing time of $150 \mu \mathrm{s},[1.8,3.0] \AA$ intervals were used. Because ${ }^{1} \mathrm{H}-{ }^{1} \mathrm{H}$ interactions are detected indirectly through the spins of bonded ${ }^{13} \mathrm{C}$ atoms, the assignment of methylene and methyl protons remains ambiguous. Following solution-state NMR spectroscopic approaches ${ }^{17}$, this aspect was accounted for during the calculation by using an $r^{-6}$ summation involving all possible ${ }^{1} \mathrm{H}-{ }^{1} \mathrm{H}$ contacts (see also reference ${ }^{9}$ ). In total, $30 \mathrm{CHHC}$ constraints were used to calculate an ensemble of 200 solid-state NMR structures. The calculations were performed twice for both possible stereospecific assignments of C22 and C23. With the assignment listed in table 4.1 the ten lowest energy structures exhibit an average total energy of $E_{\text {total }}=46 \mathrm{kcal} / \mathrm{mol}$ and an average contribution from CHHC constraint violations of $E_{\mathrm{CHHC}}=0.8 \mathrm{kcal} / \mathrm{mol}$. This shows that all $\mathrm{CHHC}$ constraints can be 
fulfilled simultaneously. In contrast, the opposite assignment of C22 and C23 leads to values of $E_{\text {total }}=61 \mathrm{kcal} / \mathrm{mol}$ and $E_{\mathrm{CHHC}}=11 \mathrm{kcal} / \mathrm{mol}$, demonstrating that it is not possible to fulfill all $\mathrm{CHHC}$ constraints simultaneously. As a result the stereospecfic assignment as listed in table 4.1 follows. Figure 4.4 shows the comparison of the 10 lowest energy solid-state NMR structures (in blue) and the X-ray structure $^{1}$ (in red). The 10 solid-state NMR structures exhibit an average heavy atom RMSD to the mean structure of $0.14 \AA$. The average heavy atom RMSD to the X-ray structure is $0.75 \AA$.

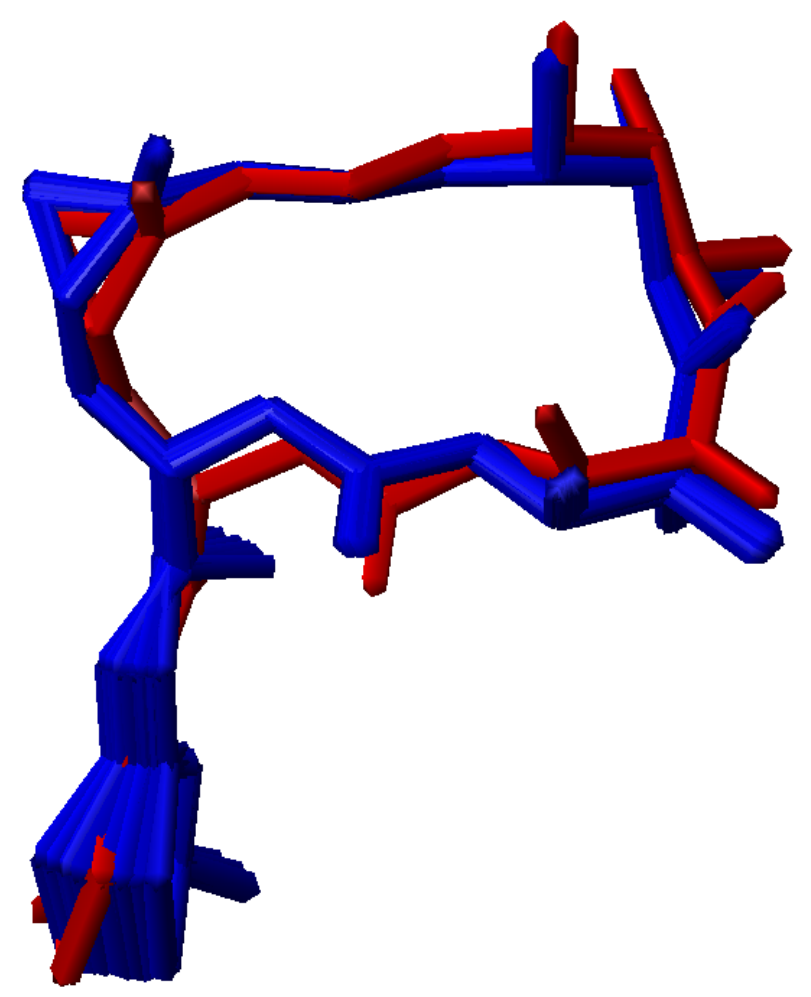

Figure 4.4. Comparison of 10 lowest energy solid-state NMR structures derived from CHHC data (shown in blue) and the X-ray structure by Höfle et al. ${ }^{1}$ (shown in red). Molecules were aligned using MOLMOL 2K. $2^{16}$.

It is informative to compare the solid-state NMR chemical shifts with solution-state NMR data obtained on epothilone $\mathrm{B}$ in $\left[\mathrm{D}_{6}\right] \mathrm{DMSO}^{1}$. As listed in table 4.1, large shift differences (i.e., $\Delta>3$ ppm) are observed for C3, C5, C6, C7, C9, C13 and C17 (see also Figure 4.5). According to Höfle et al., the conformation of the macrocycle in solution corresponds to the one found in the crystal. However, there is no preferred conformation of the thiazole side chain. For example, in solution $\mathrm{NOE}^{18}$ effects for H19-H17 and H19-H27 are observed simultaneously. This indicates a rotational 
degree of freedom of the side chain around the $\mathrm{C} 17-\mathrm{C} 18$ bond which would explain the observed chemical shift change for $\mathrm{C} 17$. Since the macrocycle is similar in solidand liquid-phase, the remaining shift changes are likely caused by the inter-molecular packing of epothilone $\mathrm{B}$ in the crystalline form. In the crystals obtained from dichloromethane (space group $P 2_{1}$ ) inter-molecular hydrogen bonds are present between $\mathrm{C} 3-\mathrm{OH}$ and the epoxide oxygen, and between $\mathrm{C} 7-\mathrm{OH}$ and the $\mathrm{C} 5$ carbonyl group $^{1}$. This would explain the observed shift changes for $\mathrm{C} 3, \mathrm{C} 13, \mathrm{C} 7$ and $\mathrm{C} 5$. The chemical shift changes for C6 and C9 might either be caused by a relay effect or by minor conformational changes that are not detected by CHHC or NOE experiments with the necessary accuracy and precision.

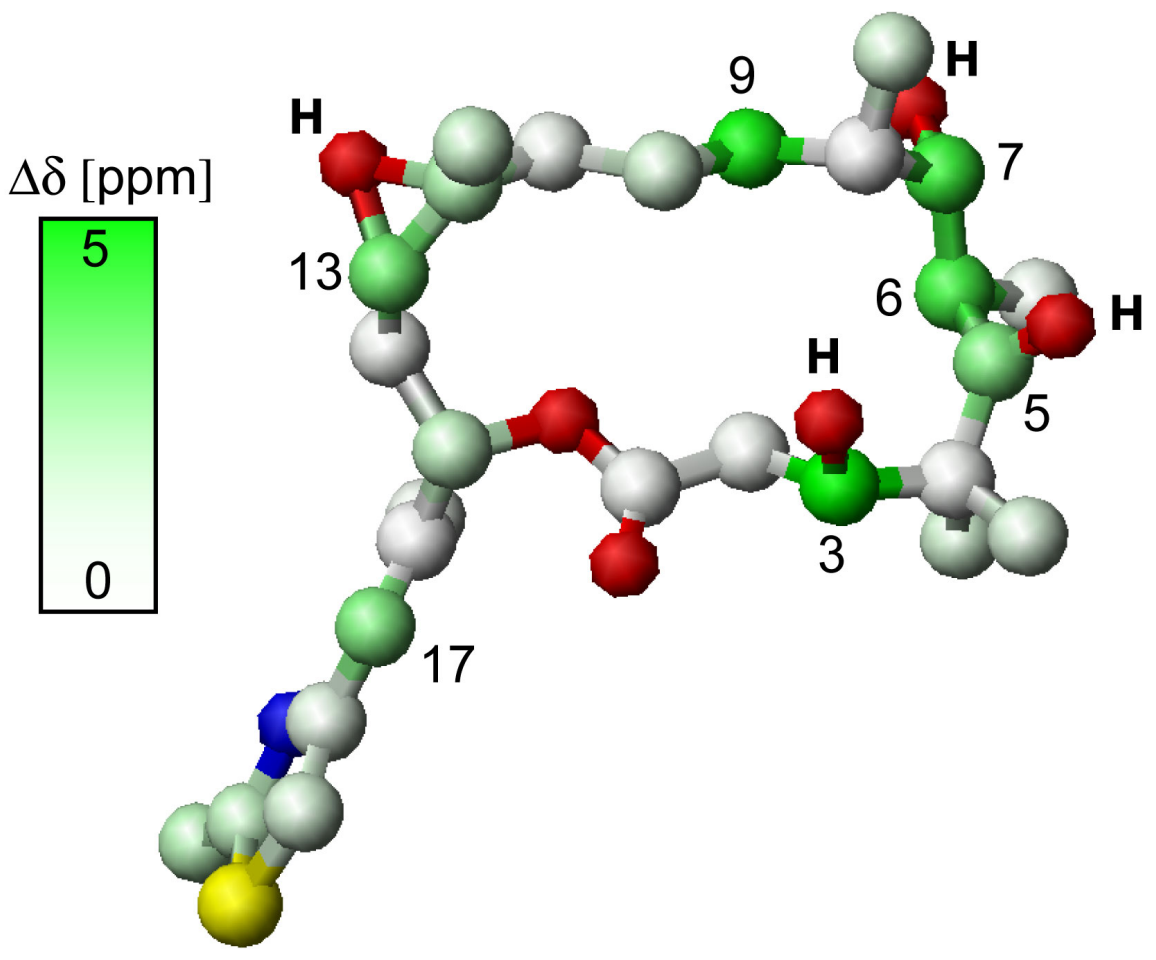

Figure 4.5. Ball and stick representation of solid-state epothilone B with the following color coding: oxygen atoms: red; nitrogen atoms: blue; sulfur atoms: yellow; carbon atoms: white to green according to chemical shift perturbation. Oxygen atoms involved in inter-molecular hydrogen bond formation are indicated with the letter $\mathrm{H}$.

\subsection{Conclusions}

The 3D structure of solid-phase epothilone B has been determined using a small set of two-dimensional solid-state NMR experiments. One ${ }^{13} \mathrm{C}$ spin diffusion experiment 
was sufficient to assign all $27{ }^{13} \mathrm{C}$ resonances unambiguously (For the stereospecific assignments of $\mathrm{C} 22$ and $\mathrm{C} 23$ also information from the CHHC spectra were used). Two CHHC experiments with mixing times in the initial rate regime yielded $30{ }^{1} \mathrm{H}-{ }^{1} \mathrm{H}$ distance constraints in the range of 1.8-3.0 $\AA$. At this mixing time relay peaks are so small that they lie below the lowest contour level in the two-dimensional contour plots and therefore do not interfere with the structural analysis. Instead of choosing very short mixing times and relatively high lowest contour levels relay peaks could also be dealt with by acquiring double-quantum $\mathrm{CHHC}^{8,9}$ spectra that allow (similar to ROESY ${ }^{19,20}$ experiments in solution) a clear classification between direct and relay peaks. Within the accuracy and precision of the experimental CHHC data one can conclude that the structure of epothilone $\mathrm{B}$ in a crystal (determined by X-ray crystallography $^{1}$ ) and as a powder (determined by solid-state NMR) are identical. This is not surprising since both samples were obtained from dichloromethane. In order to obtain a similarly large constraint set with solid-state NMR spectroscopic methods that rely on the detection of individual ${ }^{13} \mathrm{C}-{ }^{13} \mathrm{C}$ distances ${ }^{21-23}$, for each distance a build-up of two-dimensional experiments would have to be performed. Even though the $\mathrm{CHHC}$ experiment involves three $\mathrm{CP}$ transfer steps and is thus less sensitive than chemical shift selective transfer methods that involve only one $\mathrm{CP}$, the total time needed to obtain the 3D structure is drastically reduced in the CHHC approach.

The observed chemical shift differences between solid-state and solution-state chemical shifts can be explained by the rotational degrees of freedom of the thiazole side chain and the formation of inter-molecular hydrogen bonds involving oxygen atoms from the macrocycle. This indirectly confirms that the crystallites in the powder-like solid-state NMR sample are identical to the single crystal that was investigated by an X-ray crystallographic analysis ${ }^{1}$. The high sensitivity of the solidstate NMR chemical shifts to the formation of hydrogen-bonds could prove useful in the context of epothilone B / microtubule interactions. There, a comparison of chemical shifts in solution and microtubule-bound form would allow for a detection of hydrogen-bonds between epothilone $\mathrm{B}$ and polymerized $\alpha \beta$-tubulin. 
1. Höfle, G. et al. Epothilone A and B - Novel 16-membered macrolides with cytotoxic activity: Isolation, crystal structure, and conformation in solution. Angewandte Chemie-International Edition in English 35, 1567-1569 (1996).

2. Bollag, D. M. et al. Epothilones, a new class of microtubule-stabilizing agents with a taxol-like mechanism of action. Cancer Research 55, 2325-2333 (1995).

3. Kowalski, R. J., Giannakakou, P. \& Hamel, E. Activities of the microtubulestabilizing agents epothilones A and B with purified tubulin and in cells resistant to paclitaxel (taxol). Journal of Biological Chemistry 272, 2534-2541 (1997).

4. Wolff, A., Technau, A. \& Brandner, G. Epothilone A induces apoptosis in neuroblastoma cells with multiple mechanisms of drug resistance. International Journal of Oncology 11, 123-126 (1997).

5. Heinz, D. W., Schubert, W. D. \& Höfle, G. Much anticipated - The bioactive conformation of epothilone and its binding to tubulin. Angewandte ChemieInternational Edition 44, 1298-1301 (2005).

6. Carlomagno, T. et al. The high-resolution solution structure of epothilone A bound to tubulin: An understanding of the structure-activity relationships for a powerful class of antitumor agents. Angewandte Chemie-International Edition 42, 2511-2515 (2003).

7. Nettles, J. H. et al. The binding mode of epothilone A on $\alpha \beta$-tubulin by electron crystallography. Science 305, 866-869 (2004).

8. Lange, A., Luca, S. \& Baldus, M. Structural constraints from proton-mediated rare-spin correlation spectroscopy in rotating solids. Journal of the American Chemical Society 124, 9704-9705 (2002).

9. Lange, A., Seidel, K., Verdier, L., Luca, S. \& Baldus, M. Analysis of protonproton transfer dynamics in rotating solids and their use for 3D structure determination. Journal of the American Chemical Society 125, 12640-12648 (2003).

10. Lange, A. et al. A concept for rapid protein-structure determination by solidstate NMR spectroscopy. Angewandte Chemie-International Edition 44, 20892092 (2005).

11. Cornilescu, G., Delaglio, F. \& Bax, A. Protein backbone angle restraints from searching a database for chemical shift and sequence homology. Journal of Biomolecular NMR 13, 289-302 (1999).

12. Seidel, K. et al. Studying molecular 3D structure and dynamics by highresolution solid-state NMR: Application to L-tyrosine-ethylester. Journal of Physical Chemistry A 109, 2436-2442 (2005).

13. Bennett, A. E., Rienstra, C. M., Auger, M., Lakshmi, K. V. \& Griffin, R. G. Heteronuclear decoupling in rotating solids. Journal of Chemical Physics 103, 6951-6958 (1995).

14. Fung, B. M., Khitrin, A. K. \& Ermolaev, K. An improved broadband decoupling sequence for liquid crystals and solids. Journal of Magnetic Resonance 142, 97-101 (2000).

15. Brunger, A. T. et al. Crystallography \& NMR system: A new software suite for macromolecular structure determination. Acta Crystallographica Section D-Biological Crystallography 54, 905-921 (1998).

16. Koradi, R., Billeter, M. \& Wüthrich, K. MOLMOL: A program for display and analysis of macromolecular structures. Journal of Molecular Graphics 14, 51-55 (1996). 
17. Fletcher, C. M., Jones, D. N. M., Diamond, R. \& Neuhaus, D. Treatment of NOE constraints involving equivalent or non-stereoassigned protons in calculations of biomacromolecular structures. Journal of Biomolecular NMR 8, 292-310 (1996).

18. Ernst, R. R., Bodenhausen, G. \& Wokaun, A. Principles of nuclear magnetic resonance in one and two dimensions (Clarendon Press, Oxford, 1987).

19. Bothner-By, A. A., Stephens, R. L., Lee, J. M., Warren, C. D. \& Jeanloz, R. W. Structure determination of a tetrasaccharide - Transient nuclear Overhauser effects in the rotating frame. Journal of the American Chemical Society 106, 811-813 (1984).

20. Bax, A. \& Davis, D. G. Practical aspects of two-dimensional transverse NOE spectroscopy. Journal of Magnetic Resonance 63, 207-213 (1985).

21. Raleigh, D. P., Levitt, M. H. \& Griffin, R. G. Rotational resonance in solidstate NMR. Chemical Physics Letters 146, 71-76 (1988).

22. Takegoshi, K., Nomura, K. \& Terao, T. Rotational resonance in the tilted rotating frame. Chemical Physics Letters 232, 424-428 (1995).

23. Sonnenberg, L., Luca, S. \& Baldus, M. Multiple-spin analysis of chemical shift selective ${ }^{13} \mathrm{C}-{ }^{13} \mathrm{C}$ transfer in uniformly labeled biomolecules. Journal of Magnetic Resonance 166, 100-110 (2004). 


\section{Structural characterization of a high-affinity toxin / potassium channel complex}

\subsection{Introduction}

Potassium $\left(\mathrm{K}^{+}\right)$channels are part of a gene super-family of cation channels that play important roles in regulating cellular excitability. $\mathrm{K}^{+}$channels contain a conduction pore and a selectivity filter that catalyze, similar to the active site of an enzyme, the transport of $\mathrm{K}^{+}$ions across the plasma membrane ${ }^{1,2}$. Toxins from scorpion venom inhibit the active site of $\mathrm{K}^{+}$channels by occluding the pore at the extracellular opening. These scorpion toxins are characterized by a well-defined functional interaction surface from which a highly conserved lysine (K27) side chain protrudes and apparently plugs the $\mathrm{K}^{+}$channel pore ${ }^{3,4}$. Based on the conserved structures of $\mathrm{K}^{+}$ pore regions $^{5-10}$ and scorpion toxins ${ }^{3,4}$ mutagenesis and molecular modeling studies have proposed detailed structures for the $\mathrm{K}^{+}$channel / scorpion toxin binding interface. In these models and in previous solution-state NMR studies using detergentsolubilized membrane proteins ${ }^{11,12}$, scorpion toxins were docked to the extracellular entrance of the $\mathrm{K}^{+}$channel pore assuming rigid, preformed binding sites ${ }^{13-20}$. On the other hand, X-ray crystallography ${ }^{21-23}$ and MD simulations ${ }^{24}$ showed that the active site of the $\mathrm{K}^{+}$channel can adopt different conformations in response to ion binding ${ }^{25}$. Viewing the $\varepsilon$-amino group of $\mathrm{K} 27$ as a tethered $\mathrm{K}^{+}$ion $^{3}$, structural rearrangements upon toxin-channel complex formation may, likewise, be possible.

This chapter describes the usage of high-resolution solid-state nuclear magnetic resonance (ssNMR) spectroscopy to investigate directly the interaction of kaliotoxin (KTX) with the outer vestibule and the $\mathrm{K}^{+}$conduction pore of purified KcsA-Kv1.3 channels. These $\mathrm{K}^{+}$channels exhibit a similarly high affinity for $\mathrm{KTX}^{26,27}$ and $\mathrm{KTX}$ mutants (see Figure 5.5) as the Shaker-related T-lymphocyte Kv1.3 channel. Both KTX and KcsA-Kv1.3 protein were expressed in Escherichia coli and purified either in $\left[{ }^{13} \mathrm{C},{ }^{15} \mathrm{~N}\right]$ isotope-labeled or unlabeled form. Then, KTX / KcsA-Kv1.3 complexes in which only one of the constituents was isotope labeled were prepared and a series of two-dimensional ssNMR correlation experiments that probe molecular structure using chemical shift and proton-proton distance information (see, e.g., ${ }^{28}$ ) was conducted. SsNMR spectra of the complex were compared to those of both the free 
$\mathrm{KTX}^{28}$ and free KcsA-Kv1.3 channel (Fig. 5.1) in order to obtain structural information on the KTX / KcsA-Kv1.3 interaction interface.
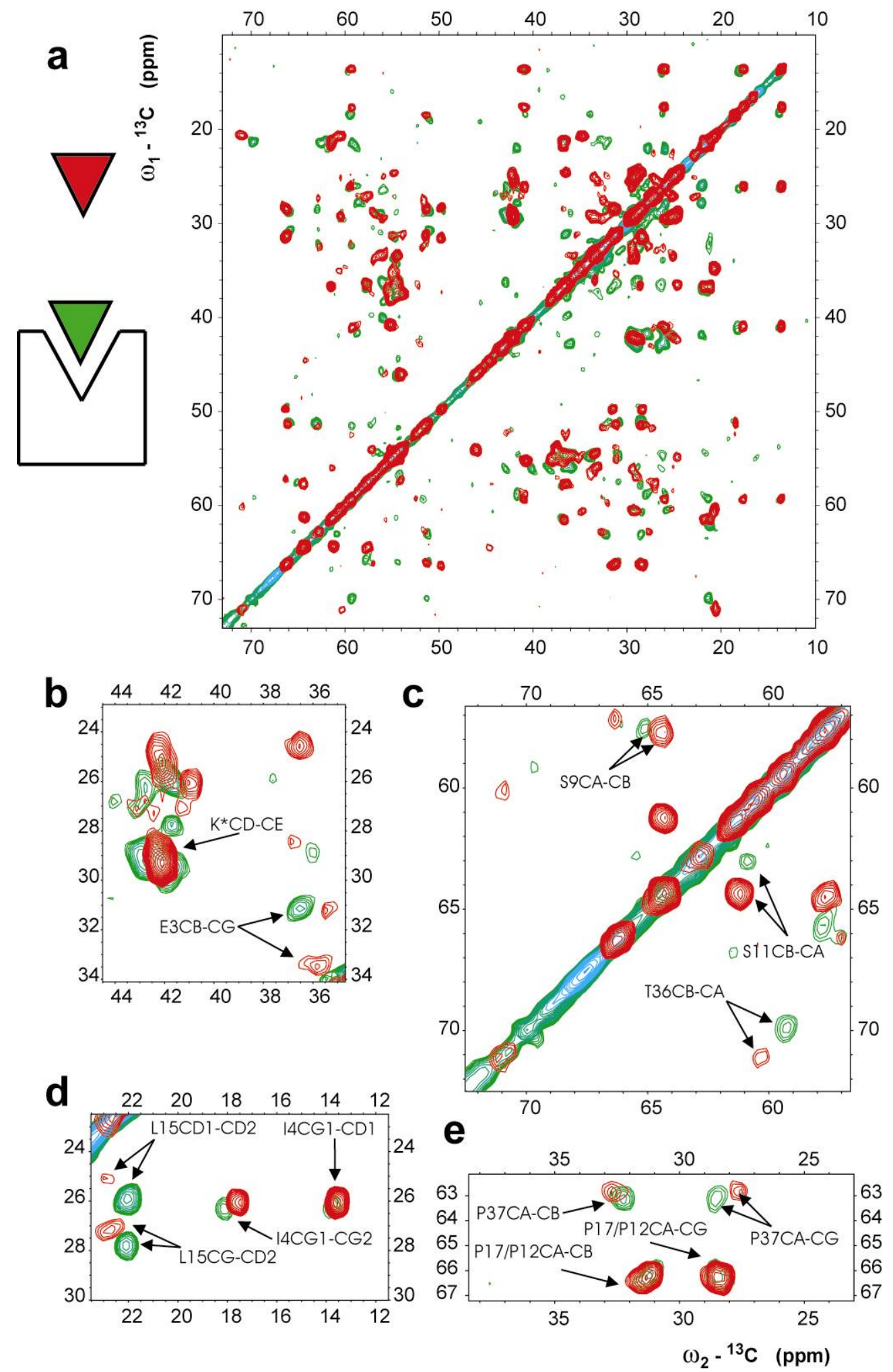

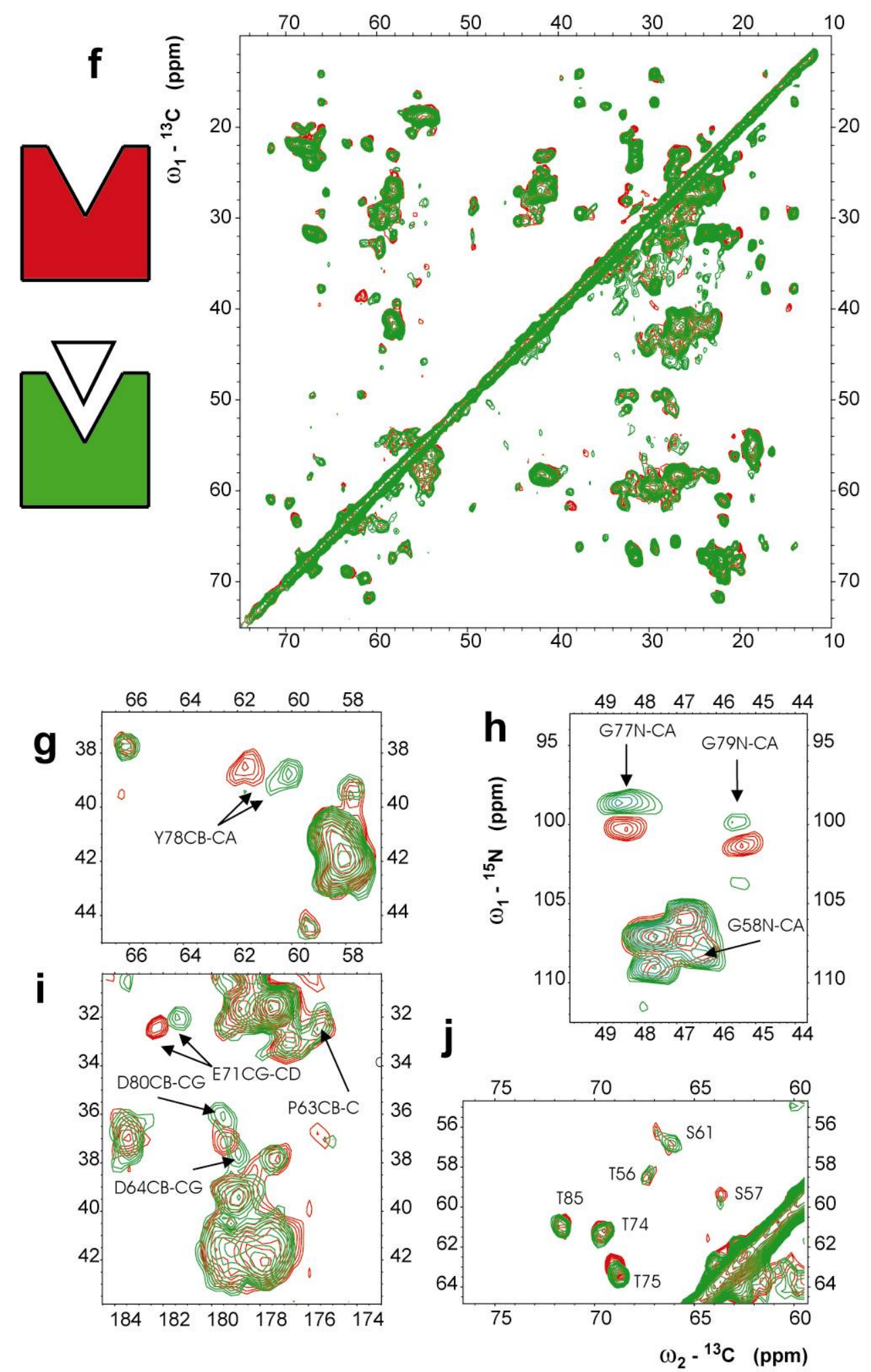

Figure 5.1. Comparison of ssNMR data of KTX and KcsA-Kv1.3 in free and complex form. ${ }^{13} \mathrm{C} 2 \mathrm{D}$ spin diffusion spectrum (a-e) obtained on uniformly $\left[{ }^{13} \mathrm{C},{ }^{15} \mathrm{~N}\right]$ labeled KTX in complex with KcsAKv1.3 (green) and in free, solid-phase form (red) as symbolized in the left column. Spectral regions are shown concerning lysine side chains (b), S11, S9 and T36 (c), methyls of L15 and I4 (d) and proline residues (e). ${ }^{13} \mathrm{C} 2 \mathrm{D}$ spin diffusion spectrum (f-j) obtained on KTX / U- $\left[{ }^{13} \mathrm{C},{ }^{15} \mathrm{~N}\right]-\mathrm{KcsA}-\mathrm{Kv} 1.3$ complex (green) and free $\mathrm{U}-\left[{ }^{13} \mathrm{C},{ }^{15} \mathrm{~N}\right]-\mathrm{KcsA}-\mathrm{Kv} 1.3$ (red). Spectral regions focus on $\mathrm{Y} 78(\mathrm{~g}), \mathrm{G} 77 / \mathrm{G} 79$ (h, NCA experiment), E71, D80, D64 (i) and T/S residues (j). 


\subsection{Sample preparation}

$\left[{ }^{13} \mathrm{C},{ }^{15} \mathrm{~N}\right]$ labeled KTX was produced as an intein fusion protein. Intein cleavage was triggered by a pH-change and the released KTX peptide was further purified by HPLC. Disulfide bond formation was verified by electrospray quadrupole mass spectrometry. Expression and purification of the KcsA-Kv1.3 chimeric channel was following the work by Legros et $\mathrm{al}^{26}$. Using a new pQE32 expression construct the channel protein was expressed in the E. coli strain M15[pREP4] at $25^{\circ} \mathrm{C}$ in 101 of superbroth medium supplemented with $0.1 \%$ glucose, ampicillin and kanamycin. Cells were disrupted with a French Press and membrane-bound protein was solubilized with $40 \mathrm{mM} n$-decyl- $\beta$-D-maltopyranoside (DM) (Calbiochem). Purification on $\mathrm{Ni}^{2+}$-nitrilotriacetic acid agarose $(6 \mathrm{ml})$ was performed as described previously $^{29}$. The channel protein was eluted with $100 \mathrm{mM}$ Na-phosphate, $\mathrm{pH}$ 7.8, 150 $\mathrm{mM} \mathrm{KCl}, 400 \mathrm{mM}$ imidazole, $4 \mathrm{mM}$ DM. The elution fractions containing the channel protein were pooled and concentrated with a Vivaspin 50.000 PES concentrator to a final volume of $9 \mathrm{ml}$. Using a HiPrep 26/10 desalting column (Amersham) the buffer was subsequently exchanged against $50 \mathrm{mM}$ Na-phosphate, pH 7.4, $50 \mathrm{mM} \mathrm{NaCl}, 4$ mM DM. The elution fractions were pooled and $\left[{ }^{13} \mathrm{C},{ }^{15} \mathrm{~N}\right]$ labeled KTX was added at a twofold molar excess. After 2 hours of incubation at room temperature, asolectin from soybean (Fluka), which had been resuspended in the same buffer ${ }^{29}$, was added at a 100/1 asolectin/KcsA-Kv1.3 molar ratio. The suspension was incubated for additional 2 hours. To remove the detergent 2 g Calbiosorb Adsorbent (Calbiochem), equilibrated with $50 \mathrm{mM}$ Na-phosphate, pH 7.4, $50 \mathrm{mM} \mathrm{NaCl}$, was added for each 66 mg DM in the suspension. The mixture was incubated on a shaker for 2 hours and loaded on a $10 \mathrm{ml}$ PIERCE column. The flowthrough as well as 3 column volumes of wash buffer fractions (50 mM Na-phosphate, $\mathrm{pH} 7.4,50 \mathrm{mM} \mathrm{NaCl}$ ) were pooled and centrifuged at $45000 \mathrm{rpm}$ (Ti50) at $4{ }^{\circ} \mathrm{C}$ for one hour.

\subsection{Electrophysiological experiments and binding studies}

${ }^{125}$ I-KTX was prepared as described previously ${ }^{30}$. In each experiment, recombinant KcsA-Kv1.3 protein (10 ng/assay) was incubated for 1 hour at room temperature in 
binding buffer containing $2 \mathrm{mM} n$-decyl- $\beta$-D-maltopyranoside with $40 \mathrm{pM}$ radiolabeled toxin and a series of cold toxin concentrations $(0.1 \mathrm{pM}$ to $1 \mu \mathrm{M})$ in a total volume of $200 \mu 1$. At the end of the incubation period, reactions were stopped by dilution with $4 \mathrm{ml}$ of wash buffer (50 mM Tris ( $\mathrm{pH} 7.4$ ), $150 \mathrm{mM} \mathrm{NaCl}, 1 \%$ bovine serum albumin). Solutions were filtered through a $\mathrm{GF} / \mathrm{C}$ glass fiber filter presoaked in $0.1 \%$ PE1. Filters were washed twice with $4 \mathrm{ml}$ of the wash buffer. Filter-retained radioactivity was determined by $\gamma$-counting. Non-specific binding was defined in presence of $1 \mu \mathrm{M}$ unlabeled toxin. In each experiment, duplicate assays were carried out, and the data were averaged from two independent experiments. Data evaluation was as described previously ${ }^{26}$.

Expression of Kv1.3 channels in Xenopus oocytes was measured one day after injection of Kv1.3 cRNA $(50 \mathrm{ng} / \mu \mathrm{l})$ in the two-electrode voltage-clamp configuration as described previously ${ }^{31}$. Bath solution was $80 \mathrm{mM} \mathrm{NaCl}, 2.0 \mathrm{mM} \mathrm{KCl}, 2.0 \mathrm{mM}$ $\mathrm{CaCl}_{2}, 1.0 \mathrm{mM} \mathrm{MgCl} 2,5.0 \mathrm{mM}$ HEPES (adjusted to $\mathrm{pH}$ 7.5). Outward currents were elicited at room temperature from $-80 \mathrm{mV}$ holding potentials to $250 \mathrm{~ms}$ long test potentials at $+60 \mathrm{mV}$. Toxins (10 $\mathrm{pM}$ to $10 \mathrm{nM}$ in bath solution containing $0.2 \%$ bovine serum albumin) were applied to scinned oocytes by bath perfusion. All experiments were repeated three to six times. Binding data were evaluated using HEKA-PULSEFIT in combination with Kaleidagraph software.

\subsection{Solid-state NMR experiments and resonance assignments}

Two-dimensional NMR experiments were conducted on a $14.1 \mathrm{~T}\left({ }^{1} \mathrm{H}\right.$ resonance frequency: $600 \mathrm{MHz}$ ) wide-bore instrument (Bruker Biospin, Germany) equipped with a $4 \mathrm{~mm}$ triple-resonance $\left({ }^{1} \mathrm{H},{ }^{13} \mathrm{C},{ }^{15} \mathrm{~N}\right)$ MAS probe. All experiments were carried out at probe temperatures of -25 to $-30{ }^{\circ} \mathrm{C}$. The MAS frequency was set to $9.375 \mathrm{kHz}$ unless stated otherwise. High power proton-decoupling using the sequence SPINAL64 ${ }^{32}$ with r.f. amplitudes of $80-90 \mathrm{kHz}$ was applied during evolution and detection periods. 
Experiments on $\mathrm{U}-\left[{ }^{13} \mathrm{C},{ }^{15} \mathrm{~N}\right]-\mathrm{KTX} / \mathrm{K} \operatorname{csA}-\mathrm{Kv} 1.3 .{ }^{13} \mathrm{C}-{ }^{13} \mathrm{C}$ : A conventional protondriven spin diffusion (SD) scheme employing longitudinal mixing times of 20, 80 and $150 \mathrm{~ms}$ was used. At $9.375 \mathrm{kHz}$ MAS, sequential CA-CA and CA-CB correlations can be detected due to weak C'-CA recoupling ${ }^{33}$. To distinguish one-bond correlations from relayed and sequential correlations, an SPC-5 double-quantum spectrum ${ }^{34}$ was recorded (MAS frequency: $7 \mathrm{kHz}$ ). For the indirect detection of ${ }^{1} \mathrm{H}-{ }^{1} \mathrm{H}$ correlations, a $\mathrm{CHHC}^{35,36}$ spectrum with a ${ }^{1} \mathrm{H}-{ }^{1} \mathrm{H}$ mixing time of $325 \mu \mathrm{s}$ was measured. Short contact times of $t_{\mathrm{HC}}=150 \mu$ s enclosing the ${ }^{1} \mathrm{H}-{ }^{1} \mathrm{H}$ transfer step ensured polarization transfer within bonded ${ }^{1} \mathrm{H}^{-13} \mathrm{C}$ pairs only. ${ }^{15} \mathrm{~N}-{ }^{13} \mathrm{C}$ : Two $\mathrm{NCA}^{37}$ spectra with different spectral windows (with and without the side chain region, respectively) and an NCACB spectrum (MAS frequency: $7 \mathrm{kHz}$ ) were recorded. In addition, NCO and NCOCA spectra were acquired. CC polarization transfer in the NCACB and NCOCA experiments was established via SD mixing for $20 \mathrm{~ms}$.

Experiments on $\mathrm{U}-\left[{ }^{13} \mathrm{C},{ }^{15} \mathrm{~N}\right]-\mathrm{K} \operatorname{cs} \mathrm{A}-\mathrm{Kv} 1.3 .{ }^{13} \mathrm{C}-{ }^{13} \mathrm{C}$ : An SD scheme employing a longitudinal mixing time of $20 \mathrm{~ms}$ was used. ${ }^{15} \mathrm{~N}-{ }^{13} \mathrm{C}$ : An NCA and an NCO spectrum were recorded. 
Table 5.1. ${ }^{13} \mathrm{C}^{15} \mathrm{~N}$ chemical shift assignments of $\mathrm{U}-\left[{ }^{13} \mathrm{C},{ }^{15} \mathrm{~N}\right]-\mathrm{KTX} / \mathrm{KcsA}-\mathrm{Kv} 1.3$.

\begin{tabular}{|c|c|c|c|c|c|c|c|c|c|}
\hline & $\mathrm{N}$ & $\mathrm{C}$ & $\mathrm{CA}$ & $\mathrm{CB}$ & $\mathrm{CG}(1)$ & $\mathrm{CG}$ & $\mathrm{CD}(1)$ & $\mathrm{CD} 2$ & $\mathrm{CE}$ \\
\hline $\mathrm{G} 1$ & & 169.8 & 43.37 & & & & & & \\
\hline $\mathrm{V} 2$ & 120 & 175 & 61.38 & 36.87 & 21.81 & 21.81 & & & \\
\hline $\mathrm{E} 3$ & & & 56.14 & 30.95 & 36.64 & & 182.9 & & \\
\hline $\mathrm{I}$ & & 176 & 59.52 & 40.98 & 26.33 & 18.23 & 13.82 & & \\
\hline V6 & 122.1 & 174.5 & 61.97 & 36.76 & 21.31 & 21.31 & & & \\
\hline $\mathrm{K} 7$ & 120.3 & & 51.91 & 37.17 & 25.56 & & & & 41.72 \\
\hline $\mathrm{C} 8$ & & 173.3 & 54.41 & 46.34 & & & & & \\
\hline S9 & 112.8 & 174.2 & 57.64 & 65.13 & & & & & \\
\hline $\mathrm{G} 10$ & 109.7 & 173.4 & 44.55 & & & & & & \\
\hline S11 & 119.9 & 173.6 & 60.81 & 62.9 & & & & & \\
\hline P12 & 139.1 & 179.4 & 65.86 & 30.76 & 28.57 & & 51.12 & & \\
\hline Q13 & 111 & & & & & & & & \\
\hline L15 & 122.1 & 179.1 & 58.79 & 41.72 & 27.84 & & 25.95 & 22.09 & \\
\hline K16 & 120.5 & 175.1 & 60.53 & 29.37 & 25.71 & & & & \\
\hline P17 & & 179.9 & 66.2 & 31.48 & 28.51 & & 49.63 & & \\
\hline C18 & 113.5 & & 57.99 & & & & & & \\
\hline D20 & & 177.3 & 56.97 & 39.47 & 178.6 & & & & \\
\hline A21 & 118.6 & 177.4 & 51.05 & 18.95 & & & & & \\
\hline G22 & 106.3 & 174.5 & 45.73 & & & & & & \\
\hline M23 & 119.6 & & 55.97 & 38.33 & & & & & 14.32 \\
\hline R24 & 120.5 & & & 35.42 & & & & & \\
\hline G26 & 109.1 & 173.9 & 45.52 & & & & & & \\
\hline K27 & 124 & & 54.57 & 35.72 & & & & & 43.07 \\
\hline C28 & 119.1 & & & & & & & & \\
\hline M29 & & 173.2 & 54.83 & 33.71 & 31.3 & & & & 16.02 \\
\hline N30 & 115.5 & & & & & & & & \\
\hline K32 & & & 54.45 & 36.08 & 26.25 & & 28.75 & & 42.79 \\
\hline C33 & 113 & 174 & 54.55 & 37.74 & & & & & \\
\hline H34 & 122.2 & & & & 130.3 & & & 117.7 & \\
\hline T36 & & 172.7 & 59.27 & 69.81 & & 21.37 & & & \\
\hline P37 & 138.7 & 176.9 & 63.06 & 32.22 & 28.48 & & 51.34 & & \\
\hline K38 & 127.3 & & & & & & & & \\
\hline
\end{tabular}


Table 5.2. Chemical shift differences $\delta$ (bound KTX) $-\delta$ (free KTX) in ppm.

\begin{tabular}{|c|c|c|c|c|c|c|}
\hline & CA & CB & CG(1) & CG2 & CD(1) & CD2 \\
\hline G1 & 0.11 & & & & & \\
\hline V2 & 0.78 & 1.96 & 1.13 & 1.13 & & \\
\hline E3 & 0.08 & -2.48 & 0.74 & & & \\
\hline I4 & 0.20 & 0.02 & 0.31 & 0.62 & 0.24 & \\
\hline N5 & & & & & & \\
\hline V6 & 0.51 & 0.02 & -0.56 & 0.12 & & \\
\hline K7 & -4.00 & 3.88 & 0.55 & & & \\
\hline C8 & 0.39 & 0.17 & & & & \\
\hline S9 & -0.04 & 0.66 & & & & \\
\hline G10 & 0.02 & & & & & \\
\hline S11 & -0.37 & -1.42 & & & & \\
\hline P12 & -0.21 & -0.43 & 0.02 & & -0.27 & \\
\hline Q13 & & & & & & \\
\hline C14 & & & & & & \\
\hline L15 & 0.37 & 0.45 & 0.73 & & 0.82 & -0.61 \\
\hline K16 & 0.07 & 0.10 & 0.52 & & & \\
\hline P17 & -0.11 & 0.05 & 0.14 & & -0.11 & \\
\hline C18 & & & & & & \\
\hline K19 & & & & & & \\
\hline D20 & & & & & & \\
\hline A21 & -0.23 & 0.53 & & & & \\
\hline G22 & 0.19 & & & & & \\
\hline M23 & 2.08 & 7.12 & & & & \\
\hline R24 & & -1.44 & & & & \\
\hline F25 & & & & & & \\
\hline G26 & -0.36 & & & & & \\
\hline K27 & & 2.22 & & & & \\
\hline C28 & & & & & & \\
\hline M29 & -0.13 & -1.66 & -0.07 & & & \\
\hline N30 & & & & & & \\
\hline R31 & & & & & & \\
\hline K32 & -0.40 & -0.47 & 1.60 & & 0.18 & \\
\hline C33 & -0.53 & -0.31 & & & & \\
\hline H34 & & & & & & \\
\hline C35 & & & & & & \\
\hline T36 & -0.90 & -1.17 & & 0.71 & & \\
\hline P37 & 0.22 & -0.35 & 1.01 & & -0.26 & \\
\hline K38 & & & & & & \\
\hline
\end{tabular}

Experiments on U- $\left[{ }^{13} \mathrm{C},{ }^{15} \mathrm{~N}\right]-\mathrm{Kcs} \mathrm{A}-\mathrm{Kv} 1.3 / \mathrm{KTX} .{ }^{13} \mathrm{C}-{ }^{13} \mathrm{C}$ : SD spectra employing longitudinal mixing times of 20,80 and 150 ms were recorded. ${ }^{15} \mathrm{~N}-{ }^{13} \mathrm{C}$ : An NCA and an NCACB spectrum (MAS frequency: $8.5 \mathrm{kHz}$ ) were recorded. In addition, NCO and NCOCA spectra were acquired. CC polarization transfer in the NCACB and NCOCA experiments was established via SD mixing for $50 \mathrm{~ms}$ and $20 \mathrm{~ms}$, 
respectively. For the indirect detection of ${ }^{1} \mathrm{H}-{ }^{1} \mathrm{H}$ correlations, a $\mathrm{NHHC}$ spectrum ${ }^{35,36}$ with a ${ }^{1} \mathrm{H}-{ }^{1} \mathrm{H}$ mixing time of $100 \mu$ s was recorded. Short contact times of $t_{\mathrm{HC}}=125 \mu \mathrm{s}$ and $t_{\mathrm{HN}}=250 \mu \mathrm{s}$, respectively, enclosing the ${ }^{1} \mathrm{H}-{ }^{1} \mathrm{H}$ transfer step ensured polarization transfer within bonded ${ }^{1} \mathrm{H}_{-}{ }^{13} \mathrm{C} /{ }^{15} \mathrm{~N}$ pairs only.

Chemical shift calibration. ${ }^{13} \mathrm{C}$ resonances were calibrated using adamantane as an external reference. The upfield resonance of adamantane was set to $31.47 \mathrm{ppm}$ to allow for a direct comparison of the solid-state chemical shifts to solution-state NMR data. Accordingly, ${ }^{15} \mathrm{~N}$ resonances were calibrated using the tripeptide $\mathrm{AGG}^{38}$ as an external reference.

Table 5.3. ${ }^{1} \mathrm{H}-{ }^{1} \mathrm{H}$ distance constraints for $\mathrm{U}-\left[{ }^{13} \mathrm{C},{ }^{15} \mathrm{~N}\right]-\mathrm{KTX} / \mathrm{KcsA}-\mathrm{Kv} 1.3$.

\begin{tabular}{cccccc}
\hline Res [\#] & RER & HB\#* & Res [\#] & & \\
11 & THR & HA & 27 & LYS & HA \\
36 & LYS & HA & 37 & PRO & HD\# \\
7 & PRO & HA & 32 & LYS & HA \\
12 & PRO & HA & 15 & LEU & HB\# \\
17 & ARG & HB\# & 20 & ASP & HB\# \\
24 & GLY & HA\# & 36 & THR & HB \\
10 & CYS & HA & 12 & PRO & HD\# \\
18 & GLY & HA\# & 23 & MET & HB\# \\
26 & LYS & HB\# & 33 & CYS & HB\# \\
16 & LYS & HA & 17 & PRO & HD\# \\
7 & LEU & HG & 32 & LYS & HG\# \\
15 & LYS & HG\# & 16 & LYS & HA \\
7 & ILE & HD1\# & 32 & LYS & HE\# \\
4 & VAL & HB & 18 & CYS & HA \\
6 & VAL & HB & 17 & PRO & HG\# \\
2 & ILE & HG1\# & 37 & PRO & HG\# \\
4 & VAL & HG\# & 33 & CYS & HB\# \\
6 & VAL & HG\# & 17 & PRO & HB\# \\
2 & VAL & HG\# & 37 & PRO & HG\# \\
6 & ILE & HG1\# & 17 & PRO & HG\# \\
4 & IGE & 6 & VAL & HG\# \\
\hline I \# indicates methyl or methylene protons & & & \\
\hline
\end{tabular}




\subsection{Structure calculations and model building}

Conformation of U- $\left[{ }^{13} \mathrm{C},{ }^{15} \mathrm{~N}\right]-\mathrm{KTX} / \mathrm{KcsA}-\mathrm{Kv} 1.3 .{ }^{13} \mathrm{CA},{ }^{13} \mathrm{CB}$ and ${ }^{15} \mathrm{NH}$ chemical shifts served as input parameters for the program TALOS version 2003.027.13.05 ${ }^{39}$ that predicts the backbone dihedral angles $\phi$ and $\psi$ based on chemical shift and sequence homology with proteins of known structure and assigned (solution-state) chemical shifts (see table 5.4). Proton-proton distance constraints were obtained by comparison of experimental and simulated CHHC spectra using the 3D structure of unbound KTX as a reference.

Structure calculations were performed with CNS (Crystallography and NMR System) version $1.1^{40} .{ }^{1} \mathrm{H}-{ }^{1} \mathrm{H}$ distance constraints obtained from the CHHC $2 \mathrm{D}$ spectrum were represented by square-well potentials with [1.8, 3.5] $\AA$ intervals. Ambiguities resulting from restraints involving methyl or methylene protons were accounted for during the calculation by using an $\mathrm{r}^{-6}$ summation involving all possible proton-proton contacts $^{41}$. Analogously, dihedral angle constraints derived from TALOS were represented by square-well potentials with upper and lower limits defined by the RMSD of the prediction. An extended conformer of kaliotoxin was created as an initial structure and was subsequently subjected to a simulated annealing as described in reference ${ }^{28}$. An ensemble of 100 structures was generated, starting from different initial velocities. The resulting structures were sorted by total energy, and the 10 lowest energy structures were selected and aligned along the backbone using MOLMOL 2K. $2^{42}$ (see table 5.5).

Conformation of the selectivity filter of $\mathrm{U}-\left[{ }^{13} \mathrm{C},{ }^{15} \mathrm{~N}\right]-\mathrm{KcsA}-\mathrm{Kv} 1.3 / \mathrm{KTX} .{ }^{13} \mathrm{CA}$ and ${ }^{13} \mathrm{CB}$ chemical shifts of the selectivity filter residues were analyzed in terms of their backbone conformation. For this purpose a grid search of the backbone angles $\phi$ and $\psi$ with the conductive structure of $\mathrm{KcsA}(1 \mathrm{~K} 4 \mathrm{C} . \mathrm{pdb})$ as a starting point was performed. Backbone angles that lead to the best agreement between calculated (with SHIFTX $^{43}$ ) and observed chemical shifts were selected. 
Table 5.4. TALOS derived dihedral angle constraints for $\mathrm{U}-\left[{ }^{13} \mathrm{C},{ }^{15} \mathrm{~N}\right]-\mathrm{KTX} / \mathrm{K} \operatorname{csA}-\mathrm{Kv} 1.3 .(\Delta \phi, \Delta \psi$ : RMSDs of TALOS predictions)

\begin{tabular}{|c|c|c|c|c|}
\hline & $\Phi$ & $\Delta \Phi$ & $\Psi$ & $\Delta \Psi$ \\
\hline $\mathrm{V} 2$ & -139 & 11 & 134 & 14 \\
\hline $\mathrm{E} 3$ & -116 & 23 & 133 & 13 \\
\hline $\mathrm{I}$ & -127 & 13 & 147 & 19 \\
\hline $\mathrm{V} 6$ & -129 & 19 & 146 & 13 \\
\hline $\mathrm{C} 7$ & -132 & 14 & 156 & 14 \\
\hline $\mathrm{S} 9$ & -139 & 8 & 156 & 9 \\
\hline $\mathrm{S} 11$ & -132 & 32 & 149 & 21 \\
\hline $\mathrm{P} 12$ & -60 & 6 & -31 & 10 \\
\hline $\mathrm{Q} 13$ & -76 & 11 & -35 & 13 \\
\hline $\mathrm{L} 15$ & -65 & 14 & -12 & 21 \\
\hline $\mathrm{K} 16$ & -60 & 7 & -44 & 4 \\
\hline $\mathrm{P} 17$ & -60 & 4 & -45 & 5 \\
\hline $\mathrm{C} 18$ & -92 & 9 & -29 & 10 \\
\hline $\mathrm{D} 20$ & -66 & 8 & -23 & 12 \\
\hline $\mathrm{A} 21$ & -86 & 12 & -2 & 10 \\
\hline $\mathrm{M} 23$ & -138 & 14 & 150 & 19 \\
\hline $\mathrm{R} 24$ & -130 & 26 & 148 & 12 \\
\hline $\mathrm{K} 27$ & -122 & 14 & 143 & 18 \\
\hline $\mathrm{C} 28$ & -123 & 23 & 145 & 18 \\
\hline $\mathrm{K} 32$ & -131 & 14 & 153 & 19 \\
\hline $\mathrm{C} 33$ & -132 & 10 & 155 & 14 \\
\hline $\mathrm{H} 34$ & -104 & 32 & 133 & 13 \\
\hline T36 & -120 & 29 & 147 & 13 \\
\hline $\mathrm{P} 37$ & -67 & 8 & 142 & 13 \\
\hline
\end{tabular}

Table 5.5. Structural statistics for 10 final structures of $\mathrm{U}-\left[{ }^{13} \mathrm{C},{ }^{15} \mathrm{~N}\right]-\mathrm{KTX} / \mathrm{KcsA}-\mathrm{Kv} 1.3$.

Energies $\left[\mathrm{kcal} \mathrm{mol}{ }^{-1}\right]^{*}$
$\mathrm{E}_{\text {total }}$
$31 \pm 3$
$E_{\text {bonds }}$
$1.0 \pm 0.2$
$E_{\text {angles }}$
$17 \pm 1$
$\mathrm{E}_{\text {impropers }}$
$1.8 \pm 0.5$
$\mathrm{E}_{\text {van der Waals }}$
$11 \pm 1$
$\mathrm{E}_{\mathrm{HH} \text { constraints }}$
$0.09 \pm 0.06$
$\mathrm{E}_{\text {dihedral angle constraints }}$
$0.7 \pm 0.2$

Average backbone RMSD to mean

$1.1 \AA$

Residues in
most favored regions
additional allowed regions
generously allowed regions
disallowed regions

$81.3 \%$

$11.7 \%$

$5.7 \%$

$1.3 \%$

of the Ramachandran plot**

[*] calculated by CNS

[**] evaluated by PROCHECK-NMR 
Table 5.6. ${ }^{13} \mathrm{C} /{ }^{15} \mathrm{~N}$ chemical shift assignments of $\mathrm{U}-\left[{ }^{13} \mathrm{C},{ }^{15} \mathrm{~N}\right]-\mathrm{Kcs} \mathrm{A}-\mathrm{Kv} 1.3$ / KTX and chemical shift differences between bound and free conformation.

U-KcsA-Kv1.3/KTX=bound

\begin{tabular}{|c|c|c|c|c|c|c|c|c|}
\hline & $\mathrm{N}$ & $\mathrm{C}$ & $\mathrm{CA}$ & $\mathrm{CB}$ & $\mathrm{CG}$ & $\mathrm{CG} 1$ & $\mathrm{CG} 2$ & $\mathrm{CD}$ \\
\hline $\mathrm{P} 55$ & & & 61.68 & 32.93 & 28.24 & & & 49.49 \\
\hline $\mathrm{T} 56$ & 122.6 & & 58.4 & 67.29 & & & 23.2 & \\
\hline $\mathrm{S} 57$ & 116.3 & 178.9 & 59.64 & 63.87 & & & & \\
\hline $\mathrm{G} 58$ & 108.3 & 176.3 & 46.33 & & & & & \\
\hline S60 & 120.3 & 175.7 & 57.84 & 64.42 & & & & \\
\hline $\mathrm{S} 61$ & 116 & 173.4 & 56.83 & 66.19 & & & & \\
\hline P63 & & 175.6 & 63.93 & 32.35 & 27.67 & & & 51.07 \\
\hline $\mathrm{D} 64$ & 117.4 & & & 37.58 & 178.9 & & & \\
\hline $\mathrm{E} 71$ & & & 59.36 & 26.54 & 32.01 & & & 181.4 \\
\hline T72 & & & 67.28 & 68.65 & & & 19.89 & \\
\hline A73 & & & 55.33 & & & & & \\
\hline T74 & 119.1 & 176.2 & 61.37 & 69.56 & & & & \\
\hline T75 & 109.9 & 172.3 & 63.43 & 68.83 & & & 21.79 & \\
\hline V76 & 121.5 & 178.2 & 66 & 32 & & 23.22 & 20.6 & \\
\hline G77 & 98.69 & 174.5 & 48.09 & & & & & \\
\hline Y78 & 112.2 & 178.4 & 60.13 & 38.84 & & & & \\
\hline G79 & 99.89 & 173.4 & 45.58 & & & & & \\
\hline D80 & & & & 35.99 & 179.6 & & & \\
\hline T85 & & 174.5 & 60.9 & 71.69 & & & 22.33 & \\
\hline
\end{tabular}

$\delta$ (bound)- $\delta$ (free)

\begin{tabular}{|l|l|l|l|l|l|l|l|l|}
\hline E71 & & & & -0.04 & -0.49 & & & -0.8 \\
\hline T74 & & & 0.17 & -0.02 & & & & \\
\hline T75 & & & 0.35 & -0.09 & & & & \\
\hline V76 & & & -0.16 & 0.37 & & 0.19 & 0.37 & \\
\hline G77 & -1.61 & & -0.23 & & & & & \\
\hline Y78 & & & -1.57 & 0.33 & & & & \\
\hline G79 & -1.51 & & 0.23 & & & & & \\
\hline
\end{tabular}

Table 5.7. ${ }^{1} \mathrm{H}-{ }^{1} \mathrm{H}$ distance constraints for $\mathrm{U}-\left[{ }^{13} \mathrm{C},{ }^{15} \mathrm{~N}\right]-\mathrm{KcsA}-\mathrm{Kv} 1.3$ / KTX.

\begin{tabular}{cccccc} 
Res [\#] & \multicolumn{5}{c}{ Res [\#] } \\
\hline 75 & THR & HN & 75 & THR & HA \\
77 & GLY & HN & 77 & GLY & HA\#* \\
76 & VAL & HN & 76 & VAL & HA \\
76 & VAL & HN & 75 & THR & HA \\
78 & TYR & HN & 76 & VAL & HB \\
77 & GLY & HN & 76 & VAL & HB \\
76 & VAL & HN & 76 & VAL & HG1\# \\
78 & TYR & HN & 78 & TYR & HA \\
78 & TYR & HN & 79 & GLY & HA\# \\
77 & GLY & HN & 76 & VAL & HA \\
\hline [*]\# indicates methyl or methylene protons & & & \\
\hline
\end{tabular}




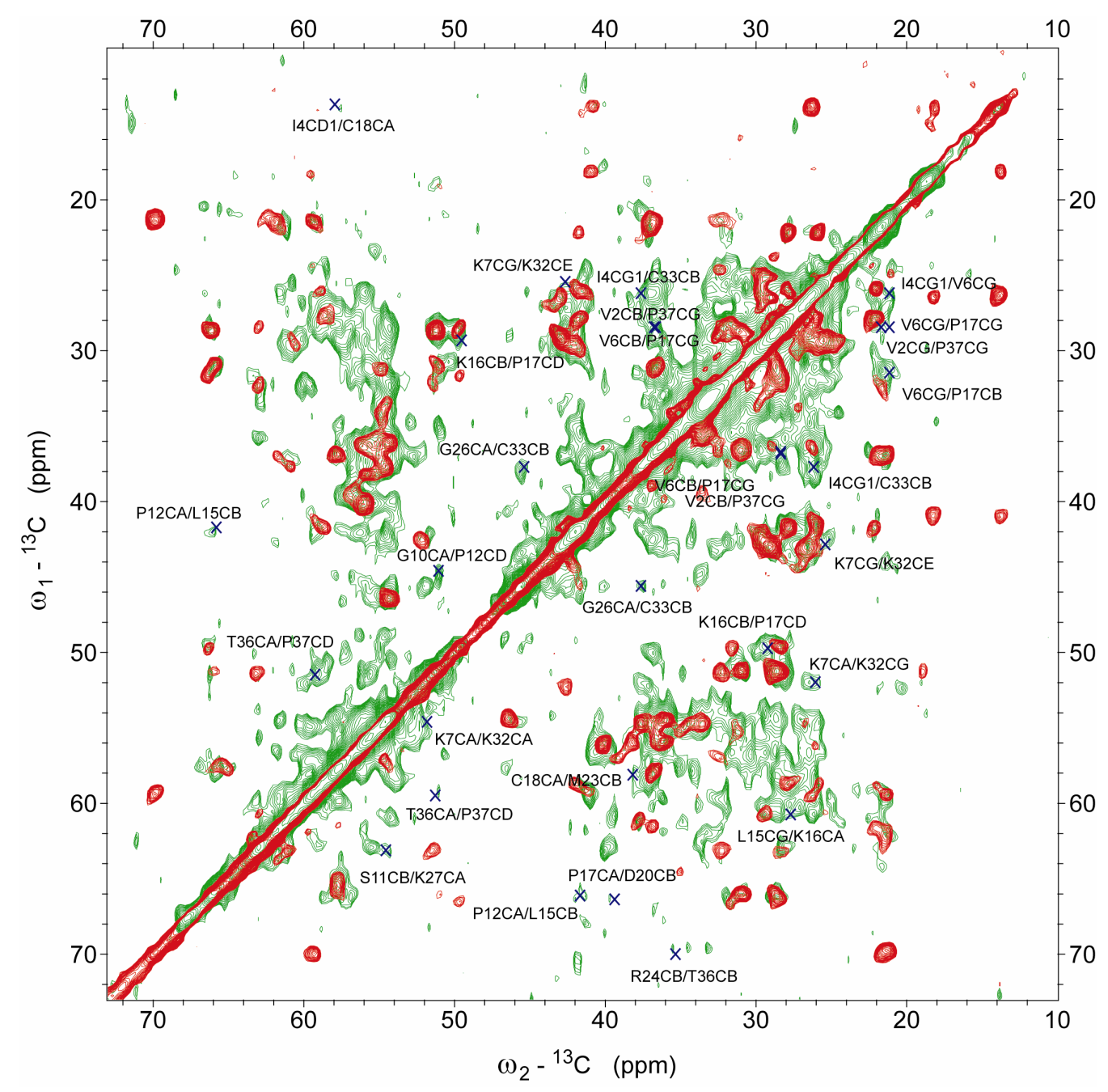

Figure 5.2. Comparison of a ${ }^{13} \mathrm{C} 2 \mathrm{D}$ spin diffusion (red) and a CHHC (green) spectrum obtained on U$\left[{ }^{13} \mathrm{C},{ }^{15} \mathrm{~N}\right]-\mathrm{KTX} / \mathrm{KcsA}-\mathrm{Kv} 1.3$. Inter-residue correlations identified in the spectrum are indicated.

\subsection{Results}

Firstly, ssNMR spectra of proteoliposomes containing purified KcsA-Kv1.3 complexed with uniformly labeled $\left[{ }^{13} \mathrm{C},{ }^{15} \mathrm{~N}\right]$-KTX were recorded. De novo sequential resonance assignments for 32 out of $38 \mathrm{KTX}$ residues were obtained (see table 5.1). As indicated in a two-dimensional ${ }^{13} \mathrm{C}-{ }^{13} \mathrm{C}$ correlation spectrum (Fig. 5.1a-e, shown in green), the ${ }^{13} \mathrm{C}$ line widths seen for isotope-labeled KTX ranged between 0.7-0.8 ppm suggesting that the KcsA-Kv1.3 toxin-receptor site is homogeneous and well-defined. A comparison to $2 \mathrm{D}{ }^{13} \mathrm{C}-{ }^{13} \mathrm{C}$ correlations observed for unbound, solid-phase $\mathrm{KTX}^{28}$ (Figure 5.1a-e, red) revealed significant $(>0.7 \mathrm{ppm}){ }^{13} \mathrm{C}$ chemical shift changes for 
several residues (see table 5.2; largest observed shift change: $\mathrm{M} 23 \mathrm{CB}: \Delta \delta=+7.12$ ppm). A secondary chemical shift analysis ${ }^{38}$ of the ssNMR data showed that bound and free KTX had a comparable backbone fold (see section 5.5). This information was used for assignments of $\mathrm{CHHC}^{35}$ correlations that are sensitive to proton-proton distances and hence to the 3D molecular structure of KTX (see table 5.3). This analysis yielded a structural model of KTX with a backbone RMSD of $1.1 \AA$ that deviated from the structure of free KTX by a backbone RMSD of $2.6 \AA$ (Figure 5.4a; see also section 5.5). Thus, the tertiary fold of KTX is conserved in the complex. However, sizeable KTX backbone chemical shift changes demonstrate that smaller structural rearrangements accompany the complex formation.

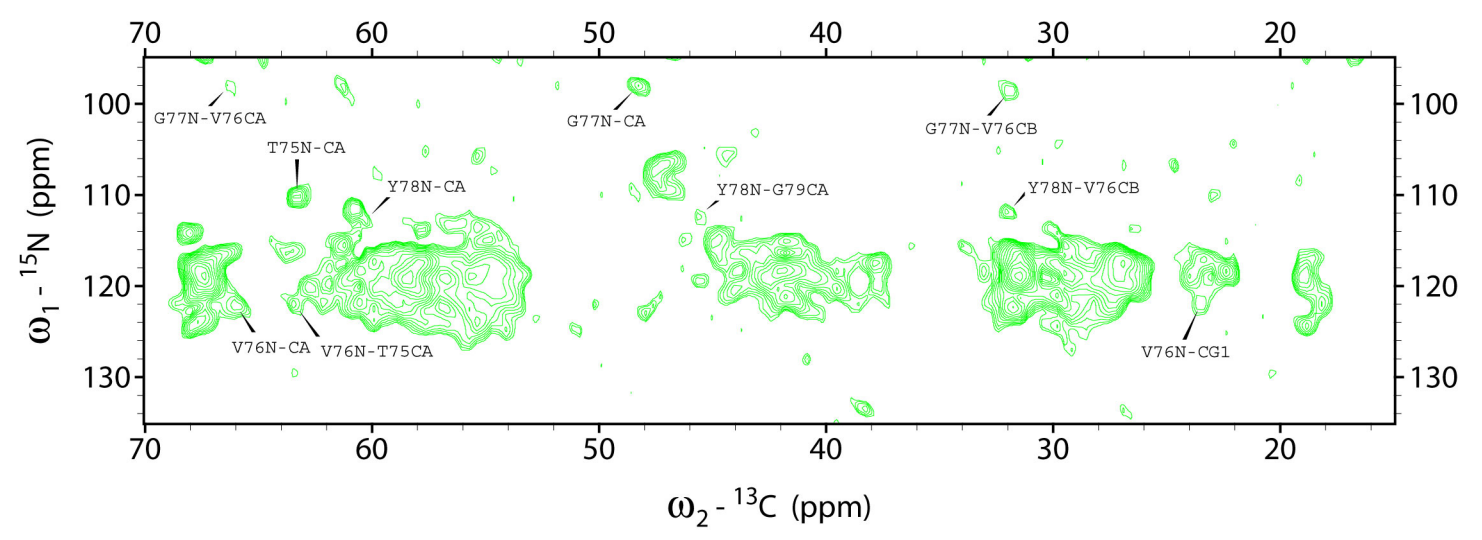

Figure 5.3. 2D NHHC spectrum of U- $\left[{ }^{13} \mathrm{C},{ }^{15} \mathrm{~N}\right]-\mathrm{KcsA}-\mathrm{Kv} 1.3$ / KTX. Assigned intra-residue and interresidue correlations involving residues 75-79 are indicated.

Previous mutagenesis studies on $\mathrm{KTX}^{15,16}$ and agitoxin ${ }^{44}$ (AgTx2) identified three toxin- $\mathrm{K}^{+}$channel interface regions, i.e., one side of the $\alpha$-helix (S11 and L15), along the second $\beta$-strand including the following turn (R24 to R31) and the end of the third $\beta$-strand (T36 and P37), respectively (Fig. 5.4a). Mutation of residues R24 and K27 crucial for KTX-binding to Kv1.3 channels $^{15,16}$ also significantly decreased KTX binding to KcsA-Kv1.3 (90-fold and 180-fold, respectively) indicating a similar functional interaction surface (Fig. 5.5). In agreement with mutagenesis and $\mathrm{MD}^{20}$ data, sizable ssNMR chemical shift changes were observed for KTX-residues S11, L15 in the $\alpha$-helix, residues M23, R24, K27, M29 along the second $\beta$-strand, and residues T36 and P37 at the end of the third $\beta$-strand (Fig. 5.4a). The data strongly supports the view that these KTX amino acids define a direct toxin- $\mathrm{K}^{+}$channel 
interaction surface with KcsA-Kv1.3. In a surface plot (Fig. 5.4a), these residues are found on one side of the KTX 3D structure in complex with the channel. On the opposite side, only residues V2, E3, and K7 exhibited sizable chemical shift changes. These residues make $\beta$-sheet contacts with the third $\beta$-strand ${ }^{15}$ and are unlikely components of the toxin- $\mathrm{K}^{+}$channel interface. Mutations of the toxin residues V2, E3 and $\mathrm{K} 7$ mildly affected both toxin inhibition of Kv1.3 currents $\left(\mathrm{IC}_{50}\right)$ expressed in Xenopus oocytes and toxin affinity to KcsA-Kv1.3 protein $\left(\mathrm{K}_{\mathrm{D}}\right)$ in competition binding assays (Fig. 5.5a and b).

a

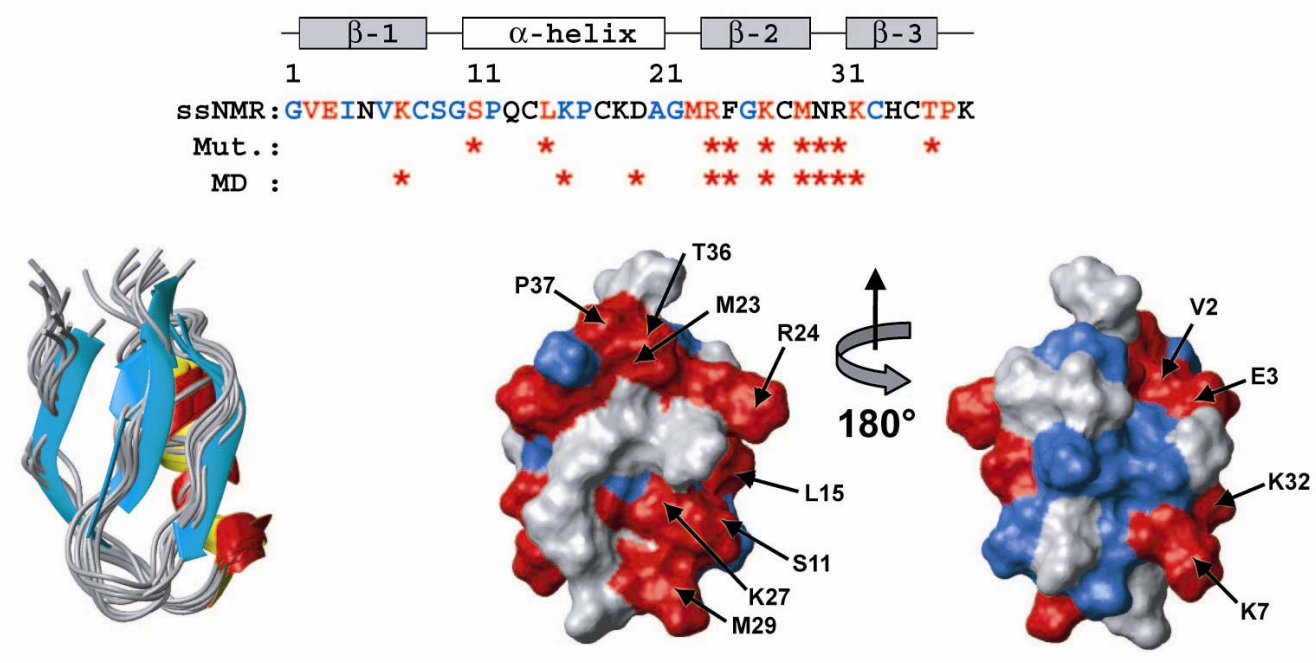

b
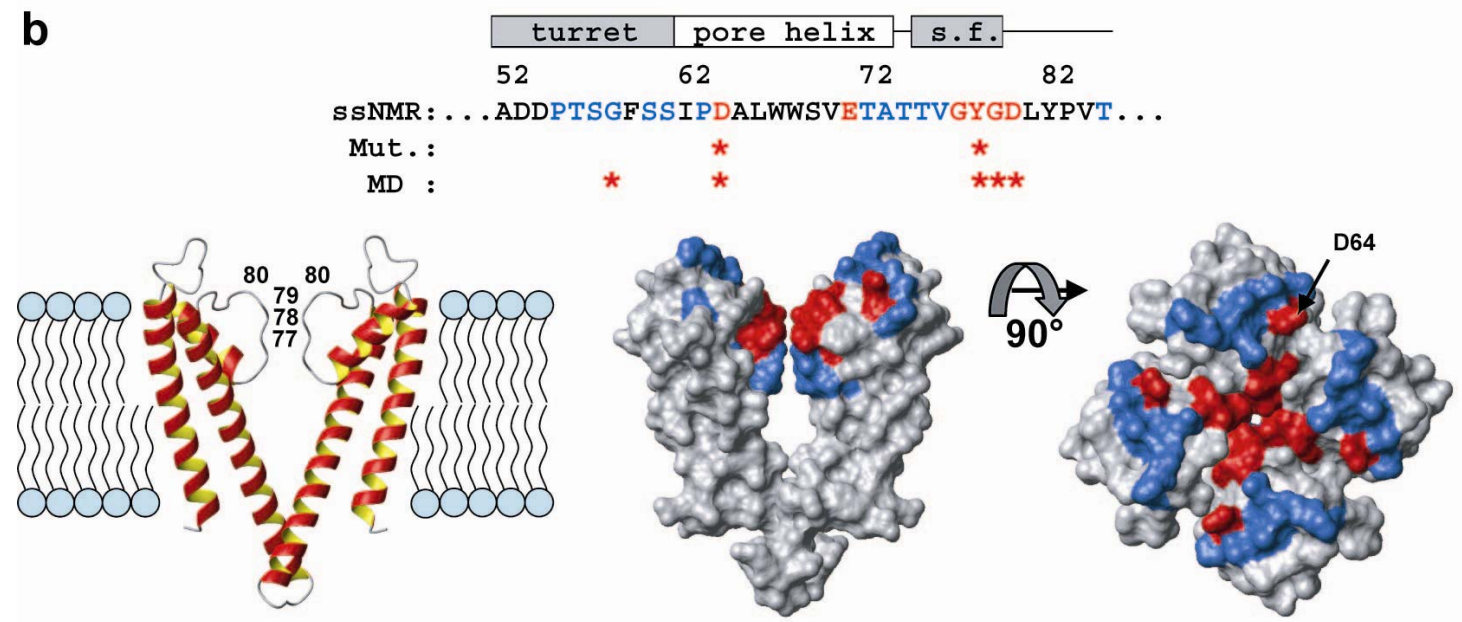

Figure 5.4. Summary of ssNMR chemical shift changes seen for KTX and KcsA-Kv1.3 upon complex formation. a,b: Comparison of ssNMR chemical shift changes seen for KTX (a) and the KcsA-Kv1.3 potassium channel (b) due to toxin binding with mutagenesis (Mut.) ${ }^{15,16}$ and modeling data (MD) ${ }^{20}$. Residues given in red relate to amino acids shown to be affected by toxin binding. Coloring in the ssNMR classification relates to residues unperturbed upon complex formation (blue) or are characterized by incomplete assignments (black). Surface plots highlight KTX (a, using the ssNMR 3D structure of KTX bound to KcsA-Kv1.3) and channel (b, using the KcsA X-ray structure; PDB entry $1 \mathrm{~K} 4 \mathrm{C}$ ) residues that are perturbed (red) or unperturbed (blue) upon complex formation. For reference, the solid-state NMR ensemble of KTX structures bound to KcsA-Kv1.3 (a) and a model of membraneinserted KcsA (b) are shown. 
Next, ssNMR spectra of isotope-labeled KcsA-Kv1.3 channel at high $(50 \mathrm{mM})$ and low $(\leq 1 \mathrm{mM}) \mathrm{KCl}$ concentrations were recorded. Analysis of these 2D ssNMR data and the observed ${ }^{13} \mathrm{C}$ line widths indicated a well-folded, largely $\alpha$-helical membrane protein in agreement with the crystal structure of conductive $\operatorname{KcsA}^{21}$ (vide infra). SsNMR measurements in the presence (green) or absence (red) of unlabeled KTX (Fig. 5.1f-j) demonstrated that the structure of outer and inner pore helices is largely maintained in KcsA-Kv1.3 after binding KTX. By contrast, chemical shift changes were seen for channel residues in the KTX-binding region. Its non- $\alpha$-helical structure facilitated ssNMR resonance assignments (see Fig. 5.4b and table 5.6).
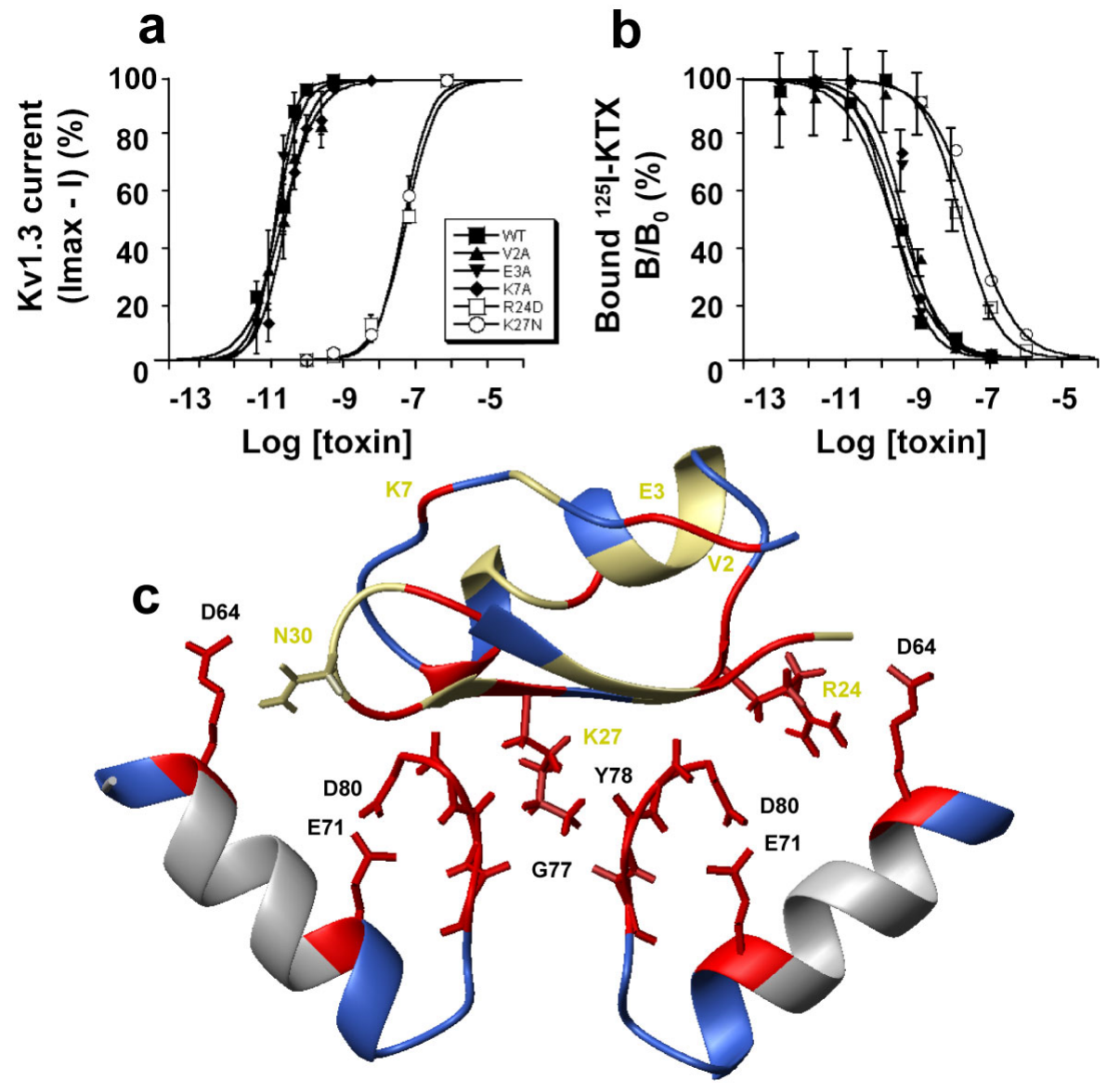

Figure 5.5. Competition binding assays of KTX mutants and structural model of the KTX / KcsAKv1.3 complex. Inhibition of Kv1.3 mediated outward-currents (a) and binding affinity to KcsA-Kv1.3 channels (b) for KTX mutants as indicated in the boxed frame. c: Model of the KTX / KcsA-Kv1.3 complex. Residues affected or unperturbed by complex formation (according to solid-state NMR chemical shift mapping) are indicated in red and blue, respectively. Information from earlier mutantcycles ${ }^{15,16}$ was used to position the toxin. 
Significant chemical shift changes (see table 5.6) could be observed for residues D64, E71, and G77-D80 in the pore helix and the selectivity filter (I62-D80, indicated in red, Fig. 5.4b). In Kv1.3 channels, mutation of D386 which is homologous to residue D64 in KcsA-Kv1.3, reduces KTX affinity by more than three orders of magnitude ${ }^{15,16}$. The solid-state NMR data confirmed that D64 in the vestibule of KcsA-Kv1.3 represents an important interaction site for KTX (Fig. 5.5). Chemical shift changes are seen for residues which are part of (G77-D80) or near (E71) to the $\mathrm{K}^{+}$signature motif TVGYGD (T75-D80). Notably, similar residues, i.e., E71, T72 and T75-D80 exhibit the largest backbone angle variations in the KcsA crystal structures obtained at low $\mathrm{K}^{+}$and high $\mathrm{K}^{+}$concentration, pointing to conformational flexibility in this part of the ion channel.

Large (>1.5 ppm) shift changes for G77 NH, Y78 CA, and G79 NH are indicative of conformational changes in the extra-cellular side of the selectivity filter. Previous models of toxin / ion channel complexes that assume the selectivity filter to be in its conductive conformation and that dock the $\varepsilon$-amino group of K27 close to the carbonyls of Y78 are incompatible with the observed shift change for residue G77. Further evidence for a conformational change in the selectivity filter region came from an NHHC experiment that indirectly encodes proton-proton proximities. The data (see Figure 5.3) suggested that the conformation of the KcsA-Kv1.3 selectivity filter in the presence of KTX differs from both KcsA crystal structures, with the largest discrepancies seen for residues 78 and 79. Finally, shift changes were also observed for the side chains of E71 and D80 that form carboxyl-carboxylate pairs and stabilize the selectivity filter.

Taken together, the data suggested that the selectivity filter of the KTX / KcsA-Kv1.3 complex adopts a different conformation upon KTX binding. Using the distinct relationship between protein backbone structure and ssNMR chemical shift, backbone torsion angles for residues T75-G79 were derived (see section 5.5). According to the resulting model, the backbone conformation of the selectivity filter in the unbound form agrees with the conductive conformation of KcsA. For the KTX-bound channel torsion angles were obtained that resemble the conductive structure of KcsA for 
residues 75-77 and are closer to the collapsed structure of KcsA for residues 78 and 79 (summarized in Figure 5.6).

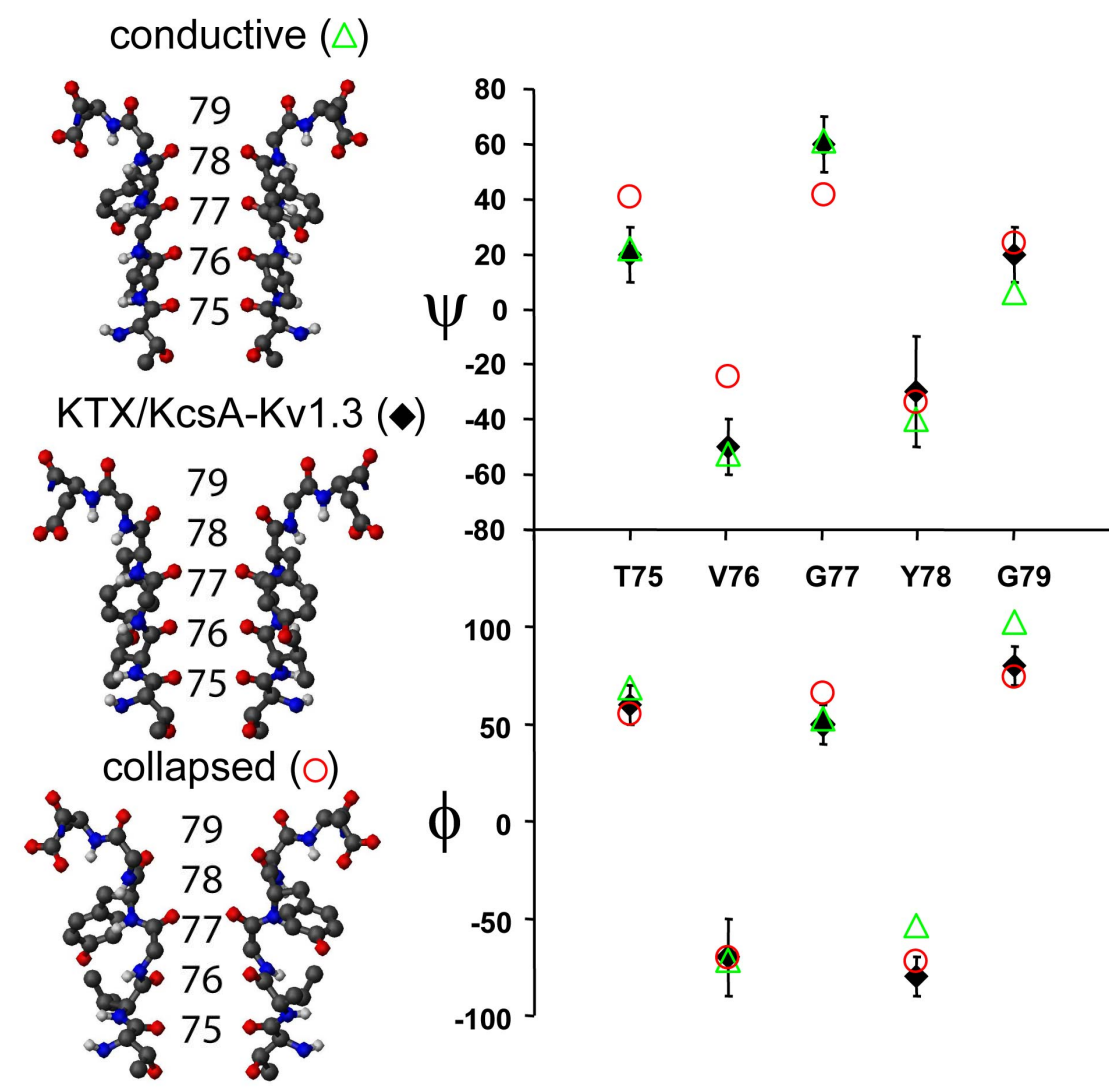

Figure 5.6. Comparison of the selectivity filter in the KTX / KcsA-Kv1.3 complex to KcsA X-ray structures. Dihedral angles derived from ssNMR chemical shifts were used to build the selectivity filter of KcsA-Kv1.3 in complex with KTX. Data are compared to X-ray structures ${ }^{21}$ obtained at high (PDB:1K4C, conductive) and low (PDB:1K4D, collapsed) $\mathrm{K}^{+}$concentrations, respectively.

\subsection{Conclusions}

Through the combination of structural and functional data a model of KcsA-Kv1.3 in complex with the scorpion toxin KTX (Figure 5.5c) is presented. The positively charged K27 $\varepsilon$-amino group comfortably fits into the KTX / KcsA-Kv1.3 selectivity filter near G77 and the hydrophobic methylene groups of the inserted K27 side chain replace water molecules in the entry region of the pore. Thus, the K27 side chain makes intimate contact to a pore region whose backbone fold is highly $\mathrm{K}^{+}$sensitive and has a different conformation in the conductive versus non-conductive state ${ }^{21}$. This view corroborates observations that $\mathrm{K} 27$ toxin variants become sensitive to the $\mathrm{K}^{+}$ 
channel's gating conformation ${ }^{45}$ and that mutations of the Y78 side chain strongly affect $\mathrm{K}^{+}$sensitivity of toxin binding to the Shaker channel, although the data shows that the Y78 side chain is not directly involved in toxin binding ${ }^{44}$.

Mutagenesis studies ${ }^{15,46}$ have indicated that residues in the turret of the P-loop region may contribute to the toxin- $\mathrm{K}^{+}$channel interaction surface, e.g., Kv1.3 residue G380 ${ }^{15,16}$ (equivalent to G58 in Fig. 5.4b). Alternatively, bulky substitutions at this glycine residue ${ }^{47}$ in the turret region may simply hinder free access of toxin to the channel binding site without directly influencing the toxin $-\mathrm{K}^{+}$channel interaction. Indeed, no chemical shift variations indicative of KTX / KcsA-Kv1.3 side chain-side chain interactions in this channel region could be observed. These findings support the structural model that high-affinity toxin binding is characterized by a deeper insertion of the toxin into the selectivity filter than previously envisioned. The data also showed that the insertion is associated with conformational changes in both molecules. It is likely that this is a prerequisite for high-affinity binding. Then, the intrinsic dynamics of the selectivity filter ${ }^{21-23}$ provide the basis for formation of a tight complex with the active site of the $\mathrm{K}^{+}$channel. A corollary of this observation is that low-affinity binding toxins ${ }^{11,12}$ may have hindered access to the active site of the $\mathrm{K}^{+}$channel and thereby fail to connect protein conformation and toxin-binding affinity. Furthermore, the structural model also explains previous observations that the toxin association rate is sensitive to the $\mathrm{K}^{+}$channel's gating conformation, but the dissociation rate is not ${ }^{48}$.

In summary, it was demonstrated that solid-state NMR spectroscopy is a sensitive method to investigate the structure of a membrane protein in complex with a peptide ligand. The results showed that the interaction between a scorpion toxin and a $\mathrm{K}^{+}$ channel is associated with structural rearrangements in different segments of the ligand and the ligand-binding site, including the selectivity filter of the channel. Taking these conformational changes into account may be crucial for a full thermodynamic characterization of high-affinity ligand binding to an ion channel. The application of solid-state NMR spectroscopy presented here can be exploited to advance current structure-based design studies leading to novel therapeutic agents in $\mathrm{K}^{+}$pharmacology. 
1. Hille, B. Ion channels of excitable membranes (Sinauer Associates Inc., Sunderland, MA, 2001).

2. Pongs, O. Voltage-gated potassium channels: From hyperexcitability to excitement. FEBS Letters 452, 31-35 (1999).

3. Miller, C. The charybdotoxin family of $\mathrm{K}^{+}$channel-blocking peptides. Neuron 15, 5-10 (1995).

4. Garcia, M. L., Gao, Y. D., McManus, O. B. \& Kaczorowski, G. J. Potassium channels: From scorpion venoms to high-resolution structure. Toxicon 39, 739-748 (2001).

5. Doyle, D. A. et al. The structure of the potassium channel: Molecular basis of $\mathrm{K}^{+}$conduction and selectivity. Science 280, 69-77 (1998).

6. Jiang, Y. X. et al. Crystal structure and mechanism of a calcium-gated potassium channel. Nature 417, 515-522 (2002).

7. Jiang, Y. X. et al. X-ray structure of a voltage-dependent $\mathrm{K}^{+}$channel. Nature 423, 33-41 (2003).

8. Jiang, Y. X., Ruta, V., Chen, J. Y., Lee, A. \& MacKinnon, R. The principle of gating charge movement in a voltage-dependent $\mathrm{K}^{+}$channel. Nature $\mathbf{4 2 3}, 42-$ 48 (2003).

9. Kuo, A. L. et al. Crystal structure of the potassium channel KirBac1.1 in the closed state. Science 300, 1922-1926 (2003).

10. Long, S. B., Campbell, E. B. \& MacKinnon, R. Crystal structure of a mammalian voltage-dependent Shaker family $\mathrm{K}^{+}$channel. Science 309, 897903 (2005).

11. Takeuchi, K. et al. Structural basis of the $\mathrm{KcsA} \mathrm{K}{ }^{+}$channel and agitoxin2 pore-blocking toxin interaction by using the transferred cross-saturation method. Structure 11, 1381-1392 (2003).

12. Yu, L. et al. Nuclear magnetic resonance structural studies of a potassium channel-charybdotoxin complex. Biochemistry 44, 15834-15841 (2005).

13. MacKinnon, R. \& Miller, C. Mutant potassium channels with altered binding of charybdotoxin, a pore-blocking peptide inhibitor. Science 245, 1382-1385 (1989).

14. Hidalgo, P. \& MacKinnon, R. Revealing the architecture of a $\mathrm{K}^{+}$channel pore through mutant cycles with a peptide inhibitor. Science 268, 307-310 (1995).

15. Aiyar, J. et al. Topology of the pore-region of a $\mathrm{K}^{+}$channel revealed by the NMR-derived structures of scorpion toxins. Neuron 15, 1169-1181 (1995).

16. Aiyar, J., Rizzi, J. P., Gutman, G. A. \& Chandy, K. G. The signature sequence of voltage-gated potassium channels projects into the external vestibule.

Journal of Biological Chemistry 271, 31013-31016 (1996).

17. MacKinnon, R., Cohen, S. L., Kuo, A. L., Lee, A. \& Chait, B. T. Structural conservation in prokaryotic and eukaryotic potassium channels. Science 280, 106-109 (1998).

18. Kalman, K. et al. ShK-Dap ${ }^{22}$, a potent Kv1.3-specific immunosuppressive polypeptide. Journal of Biological Chemistry 273, 32697-32707 (1998).

19. Eriksson, M. A. L. \& Roux, B. Modeling the structure of agitoxin in complex with the Shaker $\mathrm{K}^{+}$channel: A computational approach based on experimental distance restraints extracted from thermodynamic mutant cycles. Biophysical Journal 83, 2595-2609 (2002).

20. $\mathrm{Yu}, \mathrm{K}$. Q. et al. Computational simulations of interactions of scorpion toxins with the voltage-gated potassium ion channel. Biophysical Journal 86, 35423555 (2004). 
21. Zhou, Y. F., Morais-Cabral, J. H., Kaufman, A. \& MacKinnon, R. Chemistry of ion coordination and hydration revealed by a $\mathrm{K}^{+}$channel-Fab complex at 2.0 Å resolution. Nature 414, 43-48 (2001).

22. Zhou, Y. F. \& MacKinnon, R. The occupancy of ions in the $\mathrm{K}^{+}$selectivity filter: Charge balance and coupling of ion binding to a protein conformational change underlie high conduction rates. Journal of Molecular Biology 333, 965-975 (2003).

23. Lenaeus, M. J., Vamvouka, M., Focia, P. J. \& Gross, A. Structural basis of TEA blockade in a model potassium channel. Nature Structural \& Molecular Biology 12, 454-459 (2005).

24. Berneche, S. \& Roux, B. I. A gate in the selectivity filter of potassium channels. Structure 13, 591-600 (2005).

25. Gouaux, E. \& MacKinnon, R. Principles of selective ion transport in channels and pumps. Science 310, 1461-1465 (2005).

26. Legros, C. et al. Generating a high-affinity scorpion toxin receptor in KcsAKv1.3 chimeric potassium channels. Journal of Biological Chemistry 275, 16918-16924 (2000).

27. Legros, C. et al. Engineering specific pharmacological binding sites for peptidyl inhibitors of potassium channels into KcsA. Biochemistry 41, 1536915375 (2002).

28. Lange, A. et al. A concept for rapid protein-structure determination by solidstate NMR spectroscopy. Angewandte Chemie-International Edition 44, 20892092 (2005).

29. Cortes, D. M. \& Perozo, E. Structural dynamics of the Streptomyces lividans $\mathrm{K}^{+}$channel (SKC1): Oligomeric stoichiometry and stability. Biochemistry 36, 10343-10352 (1997).

30. Romi, R. et al. Synthesis and characterization of kaliotoxin - Is the 26-32 sequence essential for potassium channel recognition? Journal of Biological Chemistry 268, 26302-26309 (1993).

31. Stühmer, W. et al. Molecular basis of functional diversity of voltage-gated potassium channels in mammalian brain. EMBO Journal 8, 3235-3244 (1989).

32. Fung, B. M., Khitrin, A. K. \& Ermolaev, K. An improved broadband decoupling sequence for liquid crystals and solids. Journal of Magnetic Resonance 142, 97-101 (2000).

33. Seidel, K. et al. Protein solid-state NMR resonance assignments from ${ }^{13} \mathrm{C}-{ }^{13} \mathrm{C}$ correlation spectroscopy. Physical Chemistry Chemical Physics 6, 5090-5093 (2004).

34. Hohwy, M., Rienstra, C. M., Jaroniec, C. P. \& Griffin, R. G. Fivefold symmetric homonuclear dipolar recoupling in rotating solids: Application to double-quantum spectroscopy. Journal of Chemical Physics 110, 7983-7992 (1999).

35. Lange, A., Luca, S. \& Baldus, M. Structural constraints from proton-mediated rare-spin correlation spectroscopy in rotating solids. Journal of the American Chemical Society 124, 9704-9705 (2002).

36. Lange, A., Seidel, K., Verdier, L., Luca, S. \& Baldus, M. Analysis of protonproton transfer dynamics in rotating solids and their use for 3D structure determination. Journal of the American Chemical Society 125, 12640-12648 (2003). 
37. Baldus, M. Correlation experiments for assignment and structure elucidation of immobilized polypeptides under magic-angle-spinning. Progress in Nuclear Magnetic Resonance Spectroscopy 41, 1-47 (2002).

38. Luca, S. et al. Secondary chemical shifts in immobilized peptides and proteins: A qualitative basis for structure refinement under magic-angle-spinning. Journal of Biomolecular NMR 20, 325-331 (2001).

39. Cornilescu, G., Delaglio, F. \& Bax, A. Protein backbone angle restraints from searching a database for chemical shift and sequence homology. Journal of Biomolecular NMR 13, 289-302 (1999).

40. Brunger, A. T. et al. Crystallography \& NMR system: A new software suite for macromolecular structure determination. Acta Crystallographica Section D-Biological Crystallography 54, 905-921 (1998).

41. Fletcher, C. M., Jones, D. N. M., Diamond, R. \& Neuhaus, D. Treatment of NOE constraints involving equivalent or non-stereoassigned protons in calculations of biomacromolecular structures. Journal of Biomolecular NMR 8, 292-310 (1996).

42. Koradi, R., Billeter, M. \& Wüthrich, K. MOLMOL: A program for display and analysis of macromolecular structures. Journal of Molecular Graphics 14, 51-55 (1996).

43. Neal, S., Nip, A. M., Zhang, H. Y. \& Wishart, D. S. Rapid and accurate calculation of protein ${ }^{1} \mathrm{H},{ }^{13} \mathrm{C}$ and ${ }^{15} \mathrm{~N}$ chemical shifts. Journal of Biomolecular NMR 26, 215-240 (2003).

44. Ranganathan, R., Lewis, J. H. \& MacKinnon, R. Spatial localization of the $\mathrm{K}^{+}$ channel selectivity filter by mutant cycle-based structure analysis. Neuron 16, 131-139 (1996).

45. Park, C. S. \& Miller, C. Interaction of charybdotoxin with permeant ions inside the pore of a $\mathrm{K}^{+}$channel. Neuron 9, 307-313 (1992).

46. Gross, A., Abramson, T. \& MacKinnon, R. Transfer of the scorpion toxin receptor to an insensitive potassium channel. Neuron 13, 961-966 (1994).

47. Goldstein, S. A. N., Pheasant, D. J. \& Miller, C. The charybdotoxin receptor of a Shaker $\mathrm{K}^{+}$channel - Peptide and channel residues mediating molecular recognition. Neuron 12, 1377-1388 (1994).

48. Anderson, C. S., MacKinnon, R., Smith, C. \& Miller, C. Charybdotoxin block of single $\mathrm{Ca}^{2+}$-activated $\mathrm{K}^{+}$channels. Journal of General Physiology 91, 317333 (1988). 


\section{Summary}

In this thesis a general concept for three-dimensional (3D) structure determination of uniformly $\left[{ }^{13} \mathrm{C},{ }^{15} \mathrm{~N}\right]$ labeled proteins by solid-state NMR spectroscopy has been developed. This concept is based on the combination of indirectly detected ${ }^{1} \mathrm{H}-{ }^{1} \mathrm{H}$ distance constraints and chemical shift derived backbone dihedral angle constraints. The analysis of backbone ${ }^{13} \mathrm{C}$ and ${ }^{15} \mathrm{~N}$ isotropic chemical shifts in terms of polypeptide backbone conformation is a well-established technique. In contrast, the collection of multiple distance constraints in two-dimensional (2D) CHHC and NHHC experiments has only recently been introduced. Therefore, a comprehensive analysis of ${ }^{1} \mathrm{H}-{ }^{1} \mathrm{H}$ transfer in the solid-state is mandatory, if ${ }^{1} \mathrm{H}-{ }^{1} \mathrm{H}$ distance constraints are to be included in a molecular structure calculation. As described in chapter 2 of this thesis, the dependence of CHHC and NHHC cross-peak intensities on ${ }^{1} \mathrm{H}-{ }^{1} \mathrm{H}$ distance $r_{12}$, magic-angle-spinning (MAS) frequency $\omega_{r}$, static magnetic field $B_{0}$, mixing sequence, and mixing time was investigated. It was found that for mixing sequences that are characterized by a zero-quantum (0Q) average Hamiltonian (i.e., $\bar{H}_{0} \sim I_{1+} I_{2-}+I_{1-} I_{2+}$; for example RFDR or spin diffusion (SD)) the cross-peak intensities $I(t)$ exhibit an exponential buildup with the mixing time $(I(t) \sim 1-\exp (-t / T))$. The transfer rate $1 / T$ is given by the product of the 0Q lineshape function $J(\Delta)$ and the square of the ${ }^{1} \mathrm{H}-{ }^{1} \mathrm{H}$ dipolar coupling $\left(\omega_{D} / 2 \pi\right)^{2}$. Thus, in the initial rate regime of ${ }^{1} \mathrm{H}-{ }^{1} \mathrm{H}$ mixing the $\mathrm{CHHC} / \mathrm{NHHC}$ cross-peak intensity is proportional to $r_{12}^{-6} . J(\Delta)$ was found to be independent of $B_{0}$ (considered field strengths: 9.4 and $14.1 \mathrm{~T}$ ). It is strongly dependent on the MAS frequency (approximately $J(\Delta) \sim \omega_{r}^{-2}$ ) and on the mixing sequence $\left(J_{\operatorname{RFDR}}(\Delta) \cong 0.3 \cdot J_{\mathrm{SD}}(\Delta)\right)$. For mixing sequences that are characterized by a double-quantum (2Q) average Hamiltonian (i.e., $\bar{H}_{0} \sim I_{1+} I_{2+}+I_{1-} I_{2-}$; for example POST-C7) the cross-peak intensities $I(t)$ can be described within a quantum-mechanical two-spin analysis. By comparison of experimental results to quantum-mechanical simulations the ${ }^{1} \mathrm{H}-{ }^{1} \mathrm{H}$ distance of interest can be extracted. Chapter 2 also exemplifies how data from a twodimensional NHHC spectrum can be utilized to determine the structure of the model peptide Ala-Gly-Gly. Here, quantitative information for 12 distances was obtained from a single spectrum. Comparable information from experiments that are based on 
dipolar recoupling of specific ${ }^{13} \mathrm{C}-{ }^{13} \mathrm{C}$ or ${ }^{15} \mathrm{~N}-{ }^{13} \mathrm{C}$ spin pairs would have required the recording of at least 12 spectra.

In chapter 3 the concept was applied to the uniformly $\left[{ }^{13} \mathrm{C},{ }^{15} \mathrm{~N}\right]$ labeled 38 -residue peptide kaliotoxin (KTX). Three CHHC spectra with different mixing times in the initial rate regime of ${ }^{1} \mathrm{H}-{ }^{1} \mathrm{H}$ mixing were utilized to derive the tertiary fold of KTX. CHHC cross-peaks were assigned in an iterative cycle of assignment and moleculardynamics based structure calculations. Assigned CHHC correlations and chemical shift derived backbone dihedral angles (analyzed with the program TALOS) were used to determine a de novo 3D structure of KTX with an average backbone RMSD to the mean structure of $0.8 \AA$. The backbone RMSD (residues 4-38) between mean solid (PDB entry: 1XSW) and solution-state (PDB entry: 2KTX) structures was $1.9 \AA$. The solid-state NMR structure was validated by a PROCHECK-NMR analysis, which yielded $81 \%$ of all peptide residues in most favored regions of the Ramachandran plot and a back calculation of an additionally recorded NHHC 2D spectrum.

As shown in chapter 4, CHHC data can also be used to obtain 3D structures of nonpeptidic molecules. This was demonstrated on the $\left[{ }^{13} \mathrm{C}\right]$ labeled compound epothilone $\mathrm{B}$, that binds with high affinity to polymerized $\alpha \beta$-tubulin. A comparison of chemical shifts of epothilone B in solid- and liquid-phase revealed strong shift changes for the parts of the molecule that are involved in inter-molecular packing. While the experiments were performed on a powder-crystalline sample of epothilone B, they provide a basis for future studies of its bioactive conformation and binding mode to polymerized $\alpha \beta$-tubulin.

In chapter 5 the usage of high-resolution solid-state NMR spectroscopy to the investigation of high-affinity scorpion toxin / potassium channel interactions is described. A comparison of 2D spectra of the scorpion toxin KTX in free and channel (KcsA-Kv1.3) bound form revealed significant chemical shift changes for several residues. The majority of these residues lies on one side of the toxin and constitutes the toxin interaction surface. ${ }^{13} \mathrm{C}$ line widths of the channel-bound toxin in the order of $0.7 \mathrm{ppm}$ indicated that the KTX binding site of the ion channel is homogenous and well-defined. A secondary structure analysis of backbone ${ }^{13} \mathrm{C}$ and ${ }^{15} \mathrm{~N}$ chemical shifts showed that the secondary structure of KTX is conserved upon binding. However, the 
observed ${ }^{13} \mathrm{C}$ and ${ }^{15} \mathrm{~N}$ chemical shift changes point to smaller structural rearrangements in different parts of the toxin. Analysis of a $\mathrm{CHHC}$ spectrum of channel-bound toxin suggested that also the tertiary fold of KTX is conserved upon binding. By comparison of 2D spectra of free and toxin-bound ion channel the KTX binding site could be identified. Upon toxin binding sizeable backbone chemical shift variations of ${ }^{13} \mathrm{C}$ and ${ }^{15} \mathrm{~N}$ nuclei in the selectivity filter of KcsA-Kv1.3 were observed. These shift changes suggested different conformations of the selectivity filter in toxinbound and free conformation. Further evidence for this finding came from a NHHC spectrum that was recorded on toxin-bound ion channel and could not be fully explained by any published selectivity filter conformation (as determined by X-ray crystallography). The obtained structural data were combined with functional data to derive a model of the KTX / KcsA-Kv1.3 complex. This model differs from previous models of toxin / ion channel complexes that assume rigid preformed binding-sites and emphasizes the importance of structural rearrangements for toxin / membrane protein interactions. 



\section{Bibliography}

\section{Articles}

[1] A. Lange, S. Luca and M. Baldus, Structural constraints from protonmediated rare-spin correlation spectroscopy in rotating solids, Journal of the American Chemical Society, 124 (2002), pp. 9704-9705.

[2] A. Lange, K. Seidel, L. Verdier, S. Luca and M. Baldus, Analysis of protonproton transfer dynamics in rotating solids and their use for $3 D$ structure determination, Journal of the American Chemical Society, 125 (2003), pp. 12640-12648. [based on chapter 2 of this thesis]

[3] A. Böckmann, A. Lange, A. Galinier, S. Luca, N. Giraud, M. Juy, H. Heise, R. Montserret, F. Penin and M. Baldus, Solid-state NMR sequential resonance assignments and conformational analysis of the $2 \times 10.4 \mathrm{kDa}$ dimeric form of the Bacillus subtilis protein Crh, Journal of Biomolecular NMR, 27 (2003), pp. 323-339.

[4] M. Etzkorn, A. Böckmann, A. Lange and M. Baldus, Probing molecular interfaces using $2 D$ magic-angle-spinning NMR on protein mixtures with different uniform labeling, Journal of the American Chemical Society, 126 (2004), pp. 14746-14751.

[5] K. Seidel, A. Lange, S. Becker, C. E. Hughes, H. Heise and M. Baldus, Protein solid-state NMR resonance assignments from ${ }^{13} \mathrm{C}^{13} \mathrm{C}$ correlation spectroscopy, Physical Chemistry Chemical Physics, 6 (2004), pp. 5090-5093.

[6] A. Lange, S. Becker, K. Seidel, K. Giller, O. Pongs and M. Baldus, A concept for rapid protein structure determination by solid-state NMR spectroscopy, Angewandte Chemie-International Edition, 44 (2005), pp. 2089-2092. [based on chapter 3 of this thesis]

[7] A. Lange, K. Giller, S. Hornig, M. F. Martin-Eauclaire, O. Pongs, S. Becker and M. Baldus, Toxin-induced conformational changes in a potassium channel revealed by solid-state NMR, Nature, in press (2006). [based on chapter 5 of this thesis]

[8] A. Lange, T. Carlomagno and M. Baldus, The 3D solid-state NMR structure of epothilone $B$, manuscript in preparation (2006). [based on chapter 4 of this thesis]

[9] J. Korukottu, A. Lange, M. Baldus and M. Zweckstetter, Automated structure calculation based on CHHC / NHHC data, manuscript in preparation (2006).

[10] A. Lange, K. Giller, O. Pongs, S. Becker and M. Baldus, Two-dimensional solid-state NMR applied to a chimeric potassium channel, manuscript in preparation (2006). 


\section{Reviews}

[11] A. Lange and M. Baldus, Novel solid-state NMR methods for structural studies on $G$ protein-coupled receptors, $G$ protein-coupled receptors in drug discovery, Taylor and Francis, Boca Raton (2006), pp. 297-314.

[12] S. Luca, H. Heise, A. Lange and M. Baldus, Investigation of ligand / receptor systems by high-resolution solid-state NMR: Recent progress and perspectives, Archiv der Pharmazie, 338 (2005), pp. 217-228.

[13] A. Lange and M. Baldus, Membrane proteins studied by solid-state NMR, Structural biology of membrane proteins, Royal Society of Chemistry, in press (2006). 


\section{Appendix A: Two-dimensional solid-state NMR experiments}

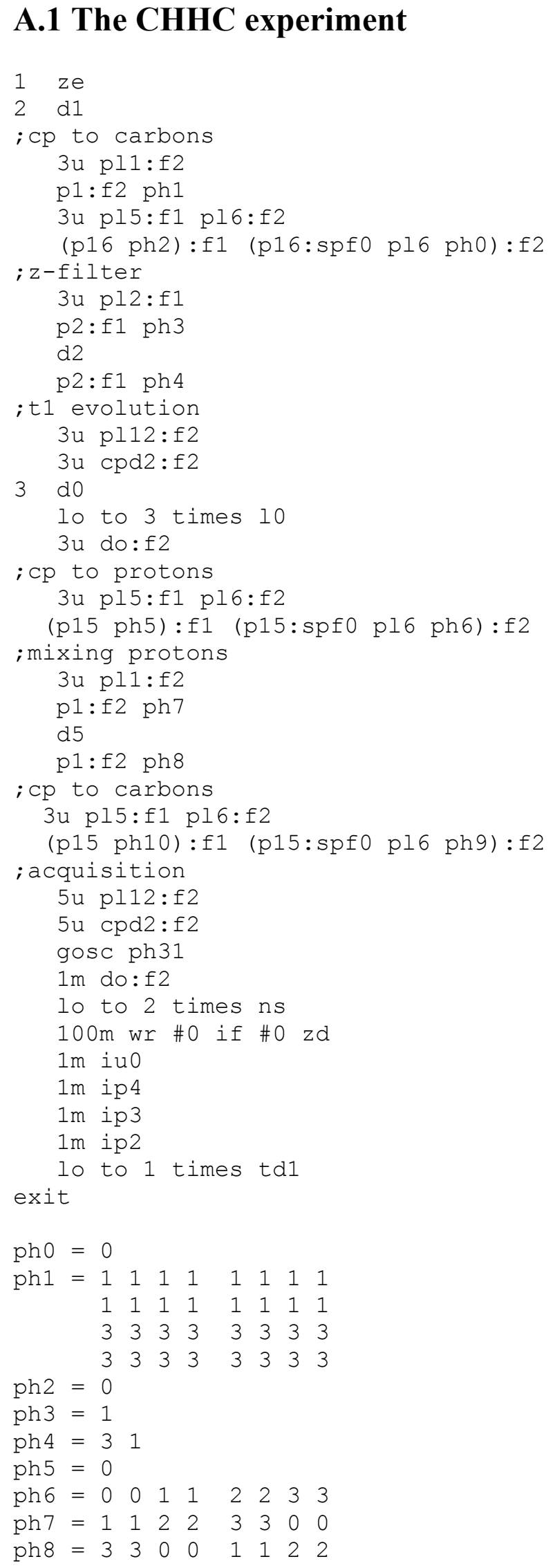


$\begin{array}{llllllll}1 & 1 & 2 & 2 & 3 & 3 & 0 & 0\end{array}$

ph $9=\begin{array}{llllllll}0 & 0 & 1 & 1 & 2 & 2 & 3 & 3\end{array}$

$\operatorname{ph} 10=\begin{array}{llllllll}0 & 0 & 1 & 1 & 2 & 2 & 3 & 3\end{array}$

$p h 31=\begin{array}{llllllll}0 & 2 & 1 & 3 & 2 & 0 & 3 & 1\end{array}$

$\begin{array}{llllllll}2 & 0 & 3 & 1 & 0 & 2 & 1 & 3\end{array}$

$\begin{array}{llllllll}2 & 0 & 3 & 1 & 0 & 2 & 1 & 3\end{array}$

$\begin{array}{llllllll}0 & 2 & 1 & 3 & 2 & 0 & 3 & 1\end{array}$ 


\section{A.2 The NHHC experiment}

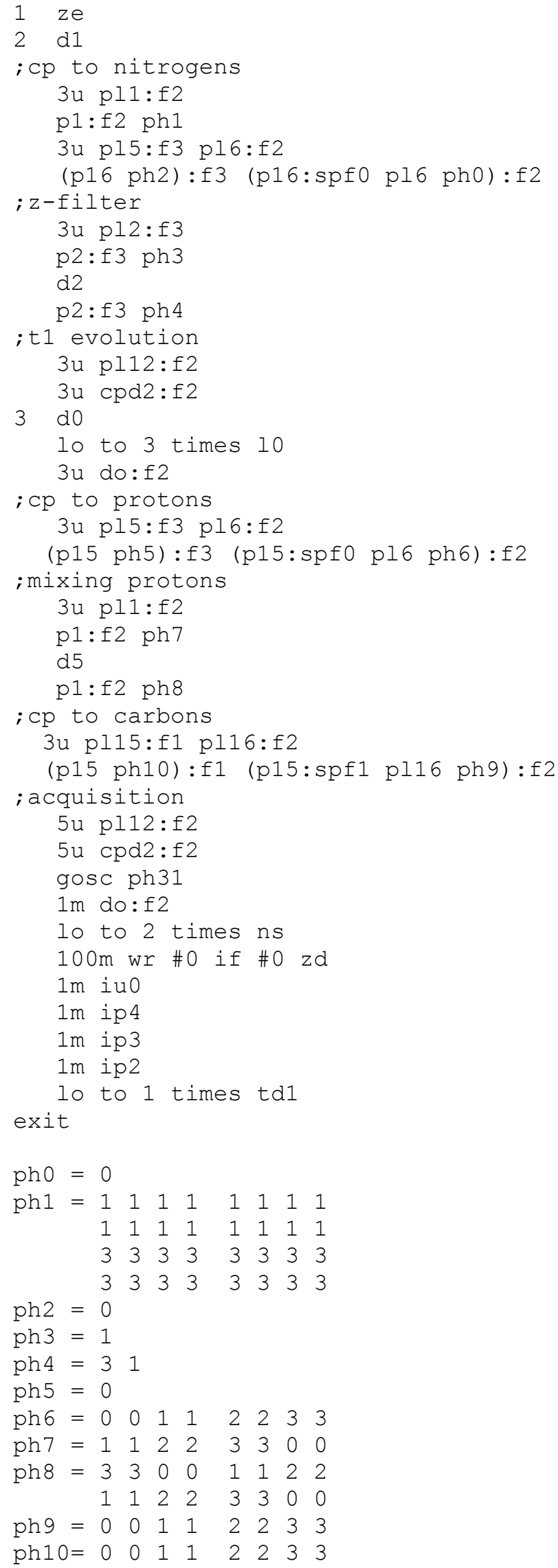


ph31= $\begin{array}{llllllll}0 & 2 & 1 & 3 & 2 & 0 & 3 & 1\end{array}$

$\begin{array}{llllllll}2 & 0 & 3 & 1 & 0 & 2 & 1 & 3\end{array}$

$\begin{array}{lllllllll}2 & 0 & 3 & 1 & 0 & 2 & 1 & 3\end{array}$

$\begin{array}{llllllll}0 & 2 & 1 & 3 & 2 & 0 & 3 & 1\end{array}$ 


\section{A.3 The 2Q-CHHC experiment}

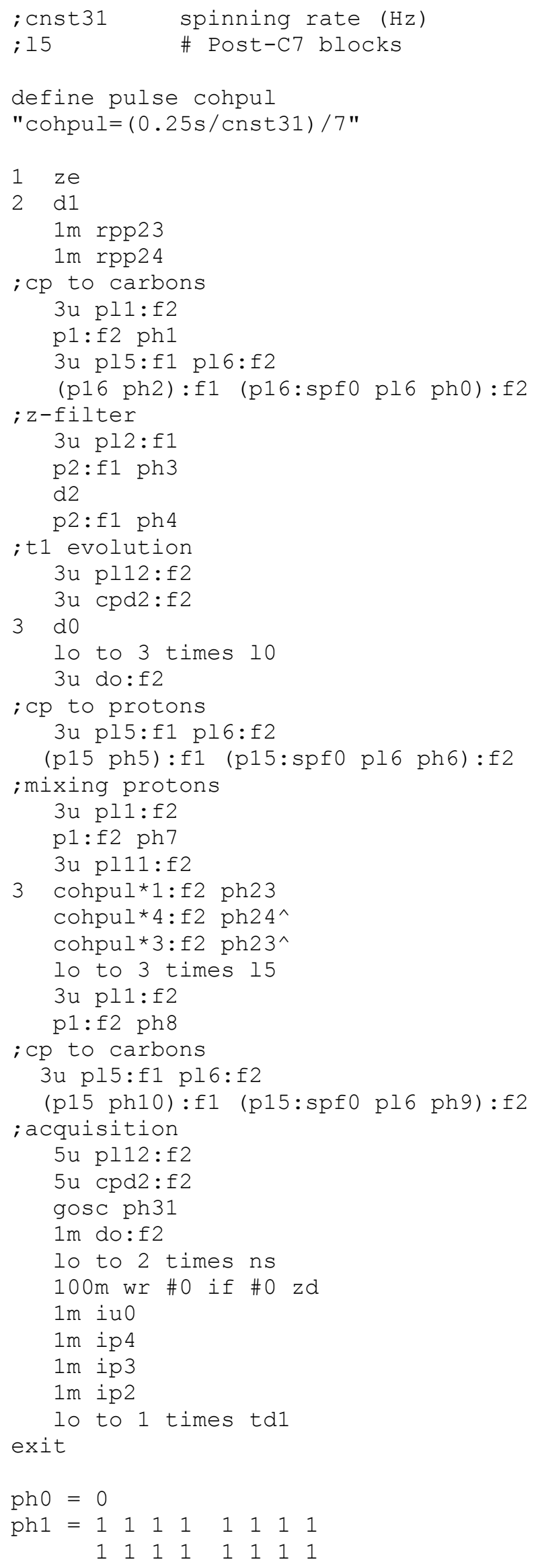




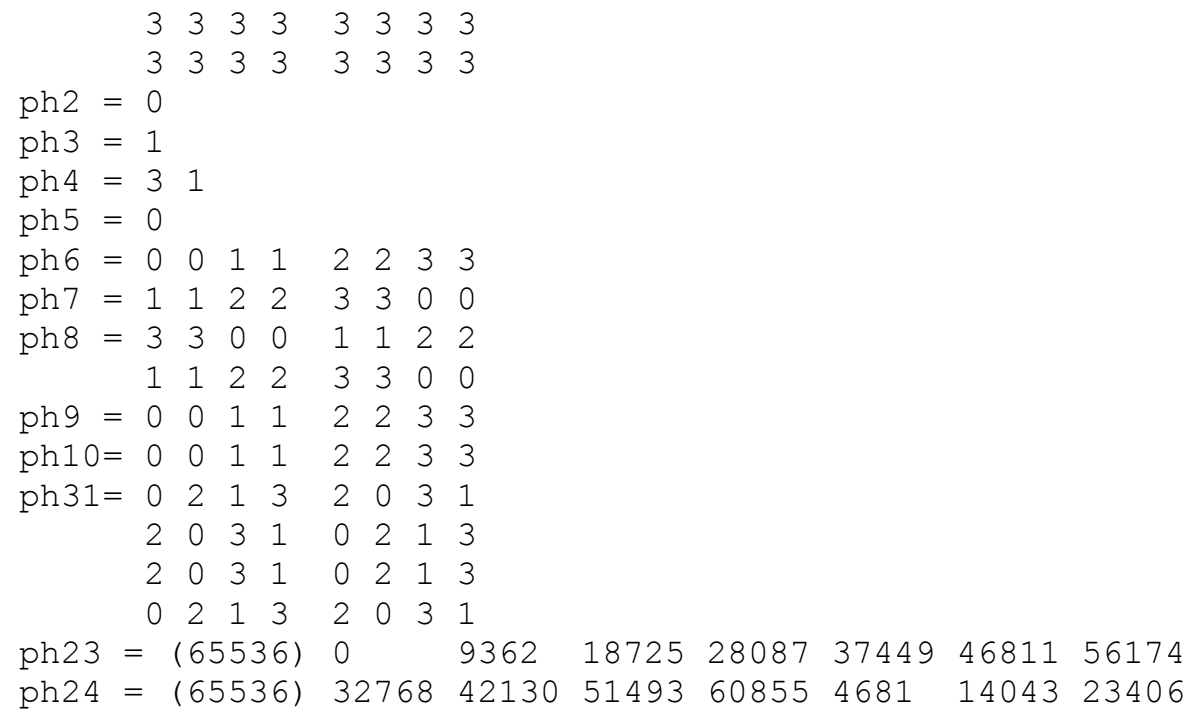




\section{A.4 The SPC-5 double-quantum experiment}

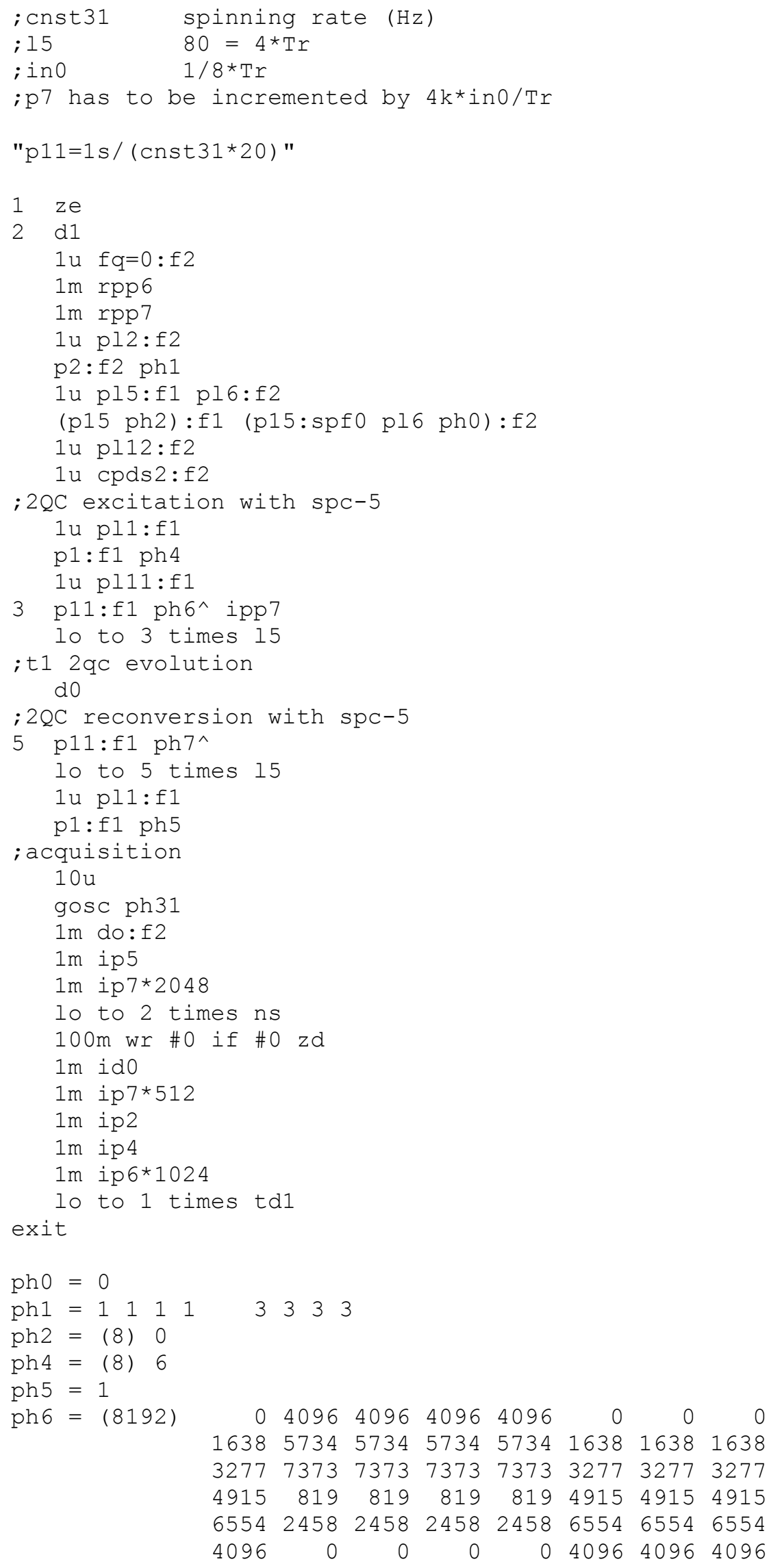




\begin{tabular}{|c|c|c|c|c|c|c|c|c|}
\hline & 5734 & 1638 & 1638 & 1638 & 1638 & 5734 & 5734 & 57 \\
\hline & 7373 & 3277 & 3277 & 3277 & 3277 & 7373 & 7373 & 737 \\
\hline & 819 & 4915 & 4915 & 4915 & 4915 & 819 & 819 & 819 \\
\hline & 2458 & 6554 & 6554 & 6554 & 6554 & 2458 & 2458 & 2458 \\
\hline $\mathrm{ph} 7=(8192)$ & & 4096 & 4096 & 4096 & 4096 & 0 & 0 & \\
\hline & 1638 & 5734 & 5734 & 5734 & 5734 & 1638 & 1638 & 163 \\
\hline & 3277 & 7373 & 7373 & 7373 & 7373 & 3277 & 3277 & 32 \\
\hline & 4915 & 819 & 819 & 819 & 819 & 4915 & 4915 & 49 \\
\hline & 6554 & 2458 & 2458 & 2458 & 2458 & 6554 & 6554 & 65 \\
\hline & 4096 & & 0 & 0 & 0 & 4096 & 4096 & 40 \\
\hline & 5734 & 1638 & 1638 & 1638 & 1638 & 5734 & 5734 & 573 \\
\hline & 7373 & 3277 & 3277 & 3277 & 3277 & 7373 & 7373 & 7373 \\
\hline & 819 & 4915 & 4915 & 4915 & 4915 & 819 & 819 & 819 \\
\hline & 2458 & 6554 & 6554 & 6554 & 6554 & 2458 & 2458 & 245 \\
\hline
\end{tabular}




\section{A.5 The NCC experiment}

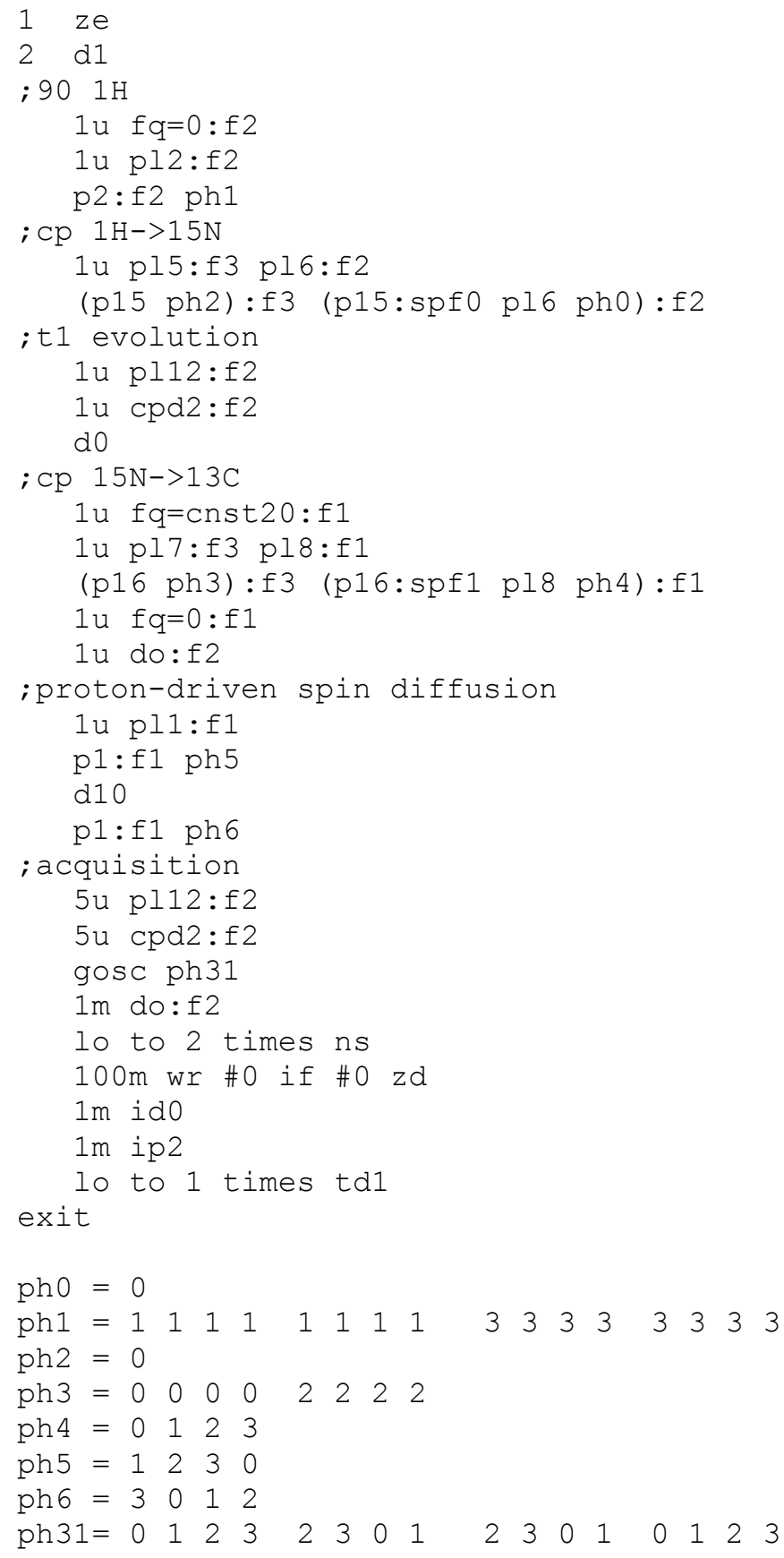




\section{A.6 The CC experiment}

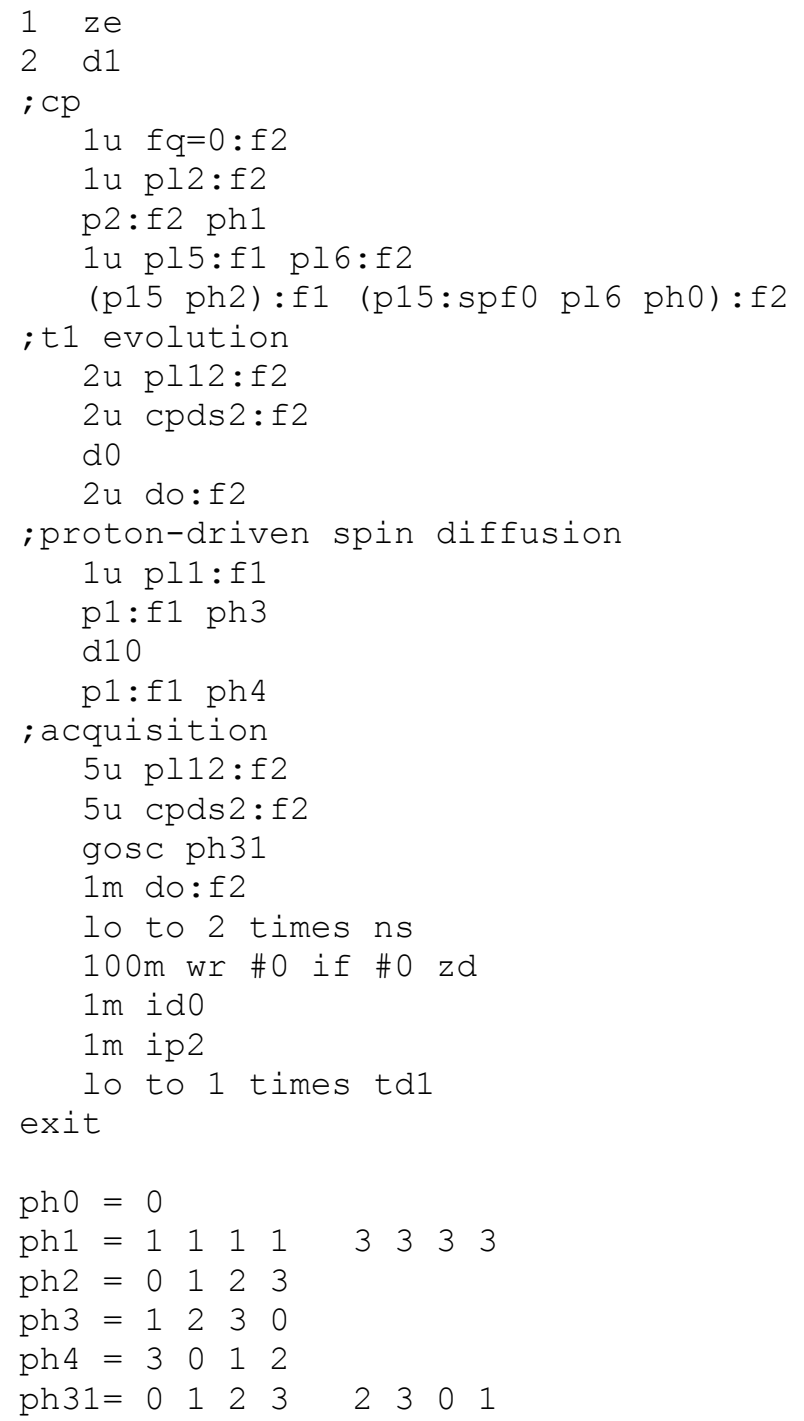




\section{Appendix B: Quantum-mechanical simulations}

\section{B.1 $\mathrm{I}_{2}$ spin system $\left(H_{\mathrm{int}}=H_{\mathrm{CSA}}+H_{\mathrm{D}}\right)$ under magic-angle-spinning}

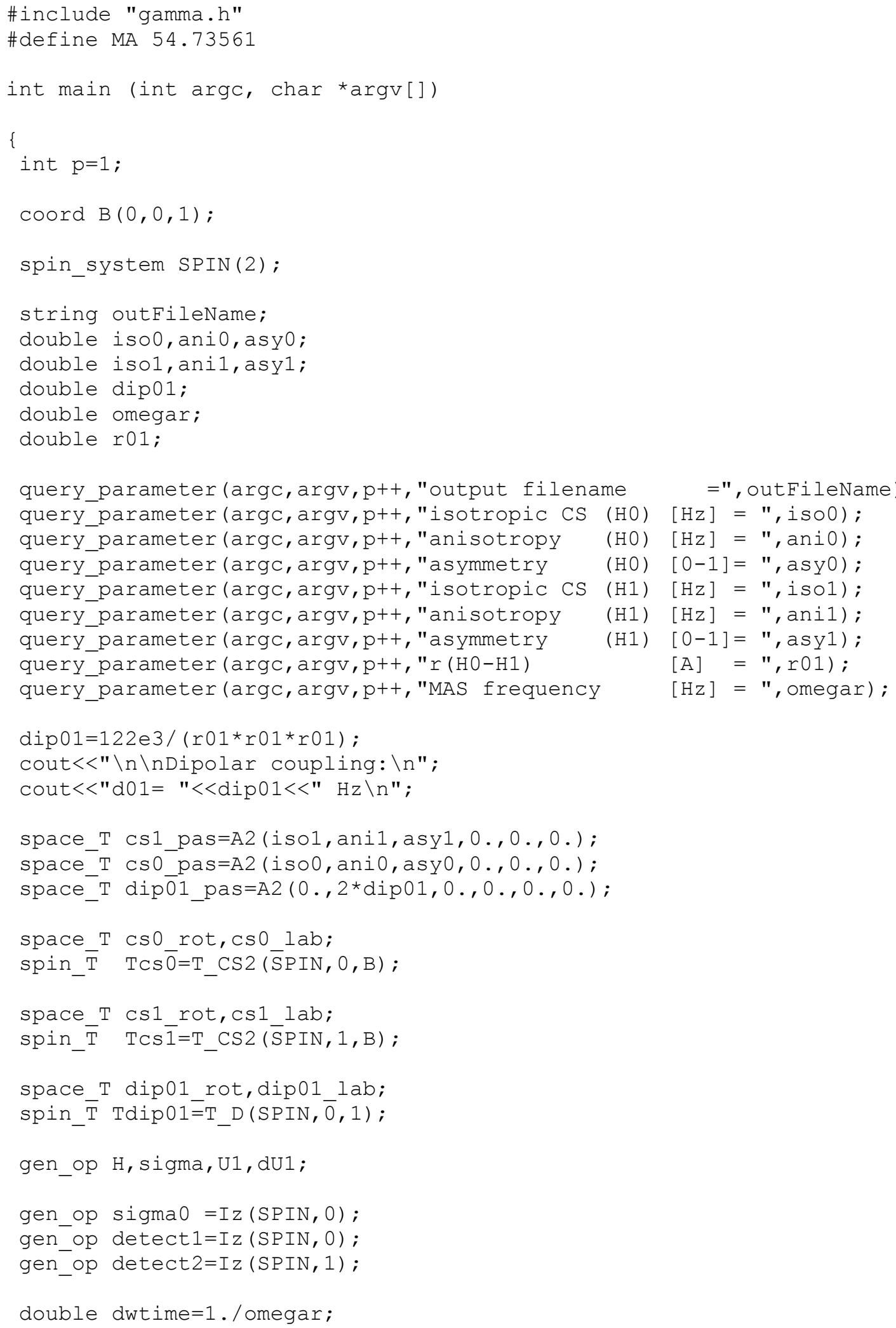




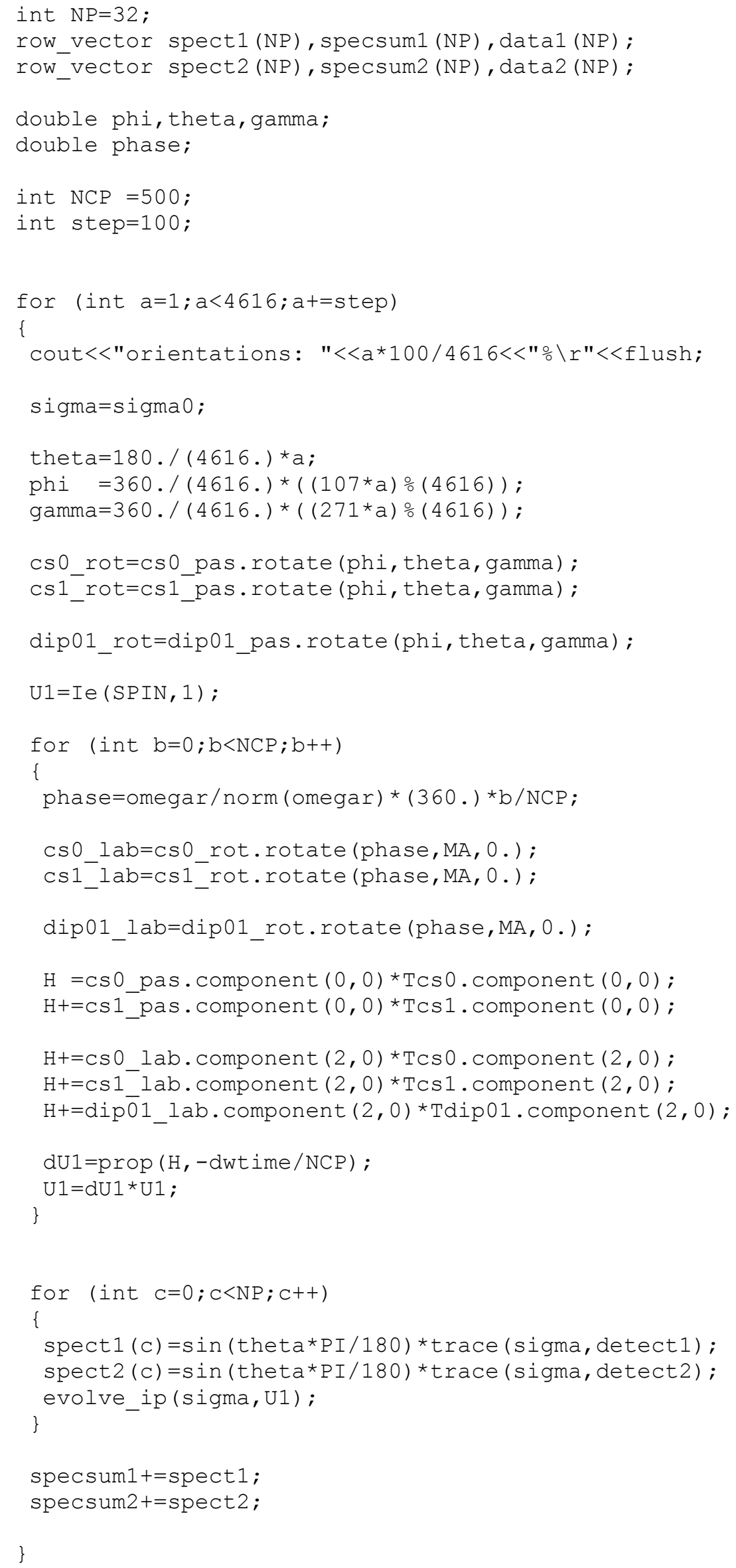




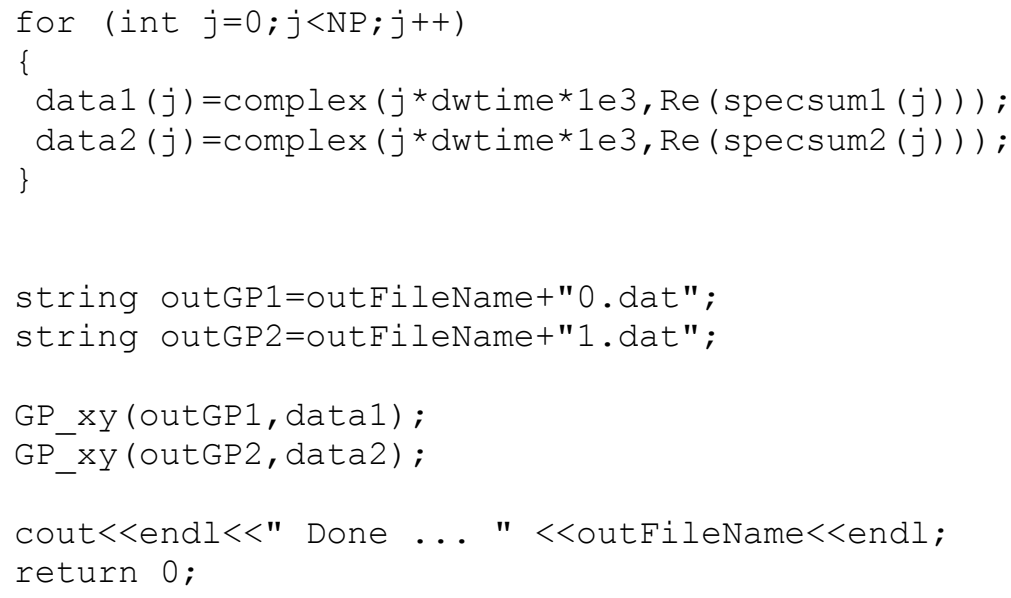





\section{Acknowledgements}

I would like to thank:

Dr. Marc Baldus for excellent supervision and the interesting projects I had the chance to work on.

Prof. Dr. Christian Griesinger for providing an outstanding scientific environment and continuous support of my projects.

Prof. Dr. Tim Salditt for accepting and supporting me as an external $\mathrm{PhD}$ student.

Dr. Reinhard Grisshammer and Dr. Susan Buchanan for giving me the opportunity to work for six months in their group at the National Institutes of Health in Bethesda, Maryland.

All current and former members of the solid-state NMR group for their continuous help and support: Ovidiu Andronesi, Gitta Angerstein, Manuel Etzkorn, Dr. Henrike Heise, Dr. Colan Hughes, Dr. Sorin Luca, Robert Schneider, and Karsten Seidel. Thank you for being such good colleagues!

Ovidiu Andronesi and Robert Schneider for the pleasant atmosphere in our office.

Karsten Seidel and Dr. Laurent Verdier for introducing me to molecular-dynamics based structure calculations and their contributions to work described in chapter two.

Karin Giller and Dr. Stefan Becker for the production of the KTX and KcsA-Kv1.3 samples that were used in chapters three and five. Without their expertise this work would not have been possible.

Prof. Dr. Olaf Pongs, Dr. Sönke Hornig, and Dr. Marie-France Eauclaire for the KTX/ KcsA-Kv1.3 collaboration and the performance of the electrophysiological experiments and binding studies that are described in chapter five.

Gitta Angerstein for the preparation of KcsA-Kv1.3 MAS samples.

Dr. Teresa Carlomagno for providing the epothilone B sample used in chapter four.

Last but not least all members of the department of NMR-based structural biology for the nice working atmosphere.

Göttingen, 10-Mar-2006 



\section{Lebenslauf}

Persönliche Daten:

Geburtsdatum: $\quad 30.05 .1977$

Geburtsort: Münster

Staatsangehörigkeit: deutsch

\section{Schule:}

1983-1987:

1987-1989:

1989-1993:

1993-1996:

11.06.1996:

Grundschule am Mönchshof, Bremen

Orientierungsstufe der Riesschule, Ritterhude

Schulverbund-Lesum, Bremen

Schulzentrum an der Bördestraße, Bremen

Abitur

\section{Zivildienst:}

07/1996 - 07/1997: im Krankenhaus der evangelischen Diakonissenanstalt Bremen

\section{Studium:}

10/1997 - 11/2002: Studium der Physik an der Georg-August-Universität Göttingen 07.11.2002:

Abschluss als Diplom-Physiker

\section{Promotion:}

11/2002 - 04/2006: Promotion am Max-Planck-Institut für Biophysikalische Chemie in Göttingen, Doktorandenstipendium des Fonds der Chemischen Industrie

\section{Auslandsaufenthalt:}

10/2003 - 04/2004: Wissenschaftlicher Mitarbeiter an den National Institutes of Health, Bethesda, Maryland, USA

Göttingen, den 10.03.2006 